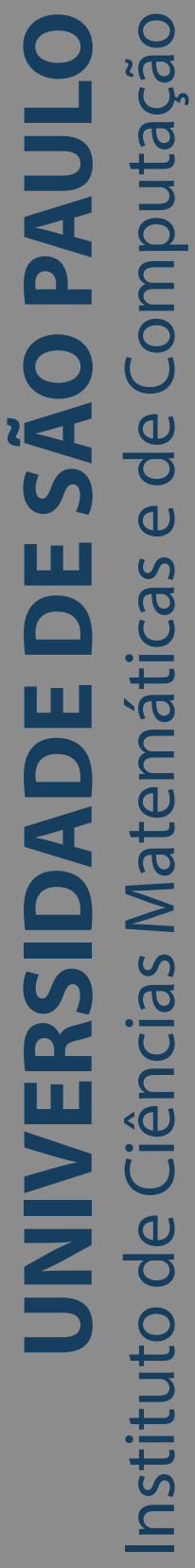

\title{
A privacy-preserving reputation scheme for trust management on VANETs applications
}

\section{Luz Marina Santos Jaimes}

Tese de Doutorado do Programa de Pós-Graduação em Ciências de Computação e Matemática Computacional (PPG-CCMC) 

Data de Depósito:

Assinatura:

\title{
Luz Marina Santos Jaimes
}

\section{A privacy-preserving reputation scheme for trust management on VANETs applications}

\begin{abstract}
Tese submitted to the Instituto de Ciências Matemáticas e de Computação - ICMC-USP, in partial fulfillment of the requirements for the degree of the Doctorate em Ciências - Ciências de Computação e Matemática Computacional. FINAL VERSION
\end{abstract}

Concentration Area: Computer Science and Computational Mathematics

Advisor: Prof. Dr. Edson dos Santos Moreira 
Ficha catalográfica elaborada pela Biblioteca Prof. Achille Bassi e Seção Técnica de Informática, ICMC/USP, com os dados fornecidos pelo(a) autor(a)

\begin{tabular}{|c|c|}
\hline \multirow[t]{3}{*}{ s237p } & $\begin{array}{l}\text { Santos Jaimes, Luz Marina } \\
\text { A privacy-preserving reputation scheme for trust } \\
\text { management on VANETs applications / Luz Marina } \\
\text { Santos Jaimes; orientador Edson dos Santos Moreira. } \\
\text {-- São Carlos, } 2017 \text {. } \\
130 \text { p. }\end{array}$ \\
\hline & $\begin{array}{l}\text { Tese (Doutorado - Programa de Pós-Graduação em } \\
\text { Ciências de Computação e Matemática Computacional) -- } \\
\text { Instituto de Ciências Matemáticas e de Computação, } \\
\text { Universidade de São Paulo, } 2017 \text {. }\end{array}$ \\
\hline & $\begin{array}{l}\text { 1. Mobile network. 2. Vehicular network. } 3 . \\
\text { Security. 4. Reputation system. } 5 \text {. Trust } \\
\text { management. I. Moreira, Edson dos Santos, orient. } \\
\text { II. Título. }\end{array}$ \\
\hline
\end{tabular}




\section{Luz Marina Santos Jaimes}

Um esquema de reputacão preservando a privacidade para o gerenciamento de confiança em aplicações VANETs

Tese apresentada ao Instituto de Ciências Matemáticas e de Computação - ICMC-USP, como parte dos requisitos para obtenção do título de Doutora em - Ciências de Computação e Matemática Computacional. VERSÃO REVISADA

Área de Concentração: Ciências de Computação e Matemática Computacional

Orientador: Prof. Dr. Edson dos Santos Moreira 

I dedicate this dissertation to my parents Julio and Alicia, husband Juan Carlos, daughter Andrea, son Juan Camilo, family and friends, for their love, comprehension and support during my Doctorate studies. 

In the first place, I thank God for giving me health, strength and granting me the capability to proceed successfully. I would like to express my gratitude to my supervisor, Professor Edson dos Santos Moreira, for his guidance, moral support, encouragement, valuable suggestions, and friendship during my Doctorate studies. I would like to thank to the administration staff and Professors of the ICMC.

I would also like to thank all my Intermídia Laboratory friends for giving me unforgettable moments and nice company during all these years. I am thankful to the São Carlos community for receiving to my family, we enjoyed wonderful years.

I am also grateful to the University of Pamplona, Colombia for providing financial support for doing my Doctorate studies.

My deepest gratitude goes to my parents, family, relative and friends who prayed for me and supported me morally throughout my studies.

Luz Marina Santos Jaimes 



\section{ABSTRACT}

SANTOS J., L. M. A privacy-preserving reputation scheme for trust management on VANETs applications. 2017. 130 p. Tese (Doutorado em Ciências - Ciências de Computação e Matemática Computacional) - Instituto de Ciências Matemáticas e de Computação, Universidade de São Paulo, São Carlos - SP, 2017.

Vehicles will use pseudonyms instead of relying on long-term certificates to provide security and privacy. Pseudonyms are short-term public key certificates that do not contain identity-linking information about the vehicle. However, there is a constant risk that authorised vehicles may send fake messages or behave selfishly, and this can affect the performance of the Vehicular Ad hoc NETwork (VANET). In this context, trust management is another important component of security services in VANETs, which provides a unified system for establishing a relationship between the nodes and helps by keeping record of the behaviour of the vehicles. Nevertheless, it is a challenging task to monitor the evolving pattern of the vehicular behaviour, since communication between the vehicles is anonymous. It is not easy to find a balanced solution that meets the requirements of security, privacy, and trust management in VANET. In view of this, we put forward a Preserving-Privacy Reputation Scheme (PPRS) applied to VANETs, in which a reputation server through a Roadside Unit receives feedback about the behaviour of the vehicles. The server updates and certifies the reputation of the vehicles by matching their anonymous identities with their real ones. Our scheme introduces geographical areas of security, in which the security of an area can be adapted to higher or lower levels depending on the reputation of the vehicles. In addition, complex reputation is examined, in which the reputation of a vehicle is linked to several behavioural factors. A further key area that is explored is the performance evaluation of PPRS which is conducted through a set of simulations in a grid scenario, based on an opportunistic message forwarding application. The results showed the effectiveness of PPRS in terms of assessing the behaviour of the vehicles and taking measures against the misbehaving vehicles. We used SUMO to simulate the mobility model; OMNET++ and Veins supported the simulation of the network model. In addition, Crypto++ was used to implement the elliptical curve cryptographic functions of signature and verification of messages, as recommended by the security standards. Finally, we employ a pseudonym changing strategy in which the reputation is discretised at two levels of reputation. The strategy was implemented in a realistic traffic simulation scenario, and was compared with the so called status and synchronous strategies through a serie of simulations. The results showed that the number of pseudonyms used in our strategy is lower than the strategies mentioned above, and maintains the rate of success of changing pseudonym achieved by the synchronous strategy.

Keywords: Mobile network, Vehicular network, Security, Reputation System, Trust management. 



\section{RESUMO}

SANTOS J., L. M. Um esquema de reputacão preservando a privacidade para o gerenciamento de confiança em aplicações VANETs. 2017. 130 p. Tese (Doutorado em Ciências Ciências de Computação e Matemática Computacional) - Instituto de Ciências Matemáticas e de Computação, Universidade de São Paulo, São Carlos - SP, 2017.

Os veículos usarão pseudônimos em vez de certificados de longo prazo para fornecer segurança e privacidade. Os pseudônimos são certificados de chaves públicas de curto prazo que não contêm informação da identidade do veículo. No entanto, existe risco que veículos autorizados possam enviar mensagens falsas ou se comportar de maneira egoísta, e isso pode afetar o desempenho das redes veiculares (VANETs). Nesse contexto, o gerenciamento de confiança é um importante serviço de segurança nas VANETs, o qual fornece um sistema unificado para estabelecer relações entre os nós e ajuda a manter um registro do comportamento dos veículos. No entanto, é uma tarefa desafiante monitorar o padrão evolutivo do comportamiento veícular, já que a comunicação entre os veículos é anônima. Não é uma tarefa fácil encontrar uma solução equilibrada que atenda aos requisitos de segurança, privacidade e gerenciamento de confiança em VANET. Em vista disso, apresentamos um Esquema de Reputação Preservando a Privacidade (ERPP) aplicado a VANETs, no qual um servidor de reputação através de uma unidade de acostamento recebe avaliações sobre o comportamento dos veículos. O servidor atualiza e certifica a reputação dos veículos relacionando seus identidades anônimas com as reais. ERPP introduz áreas geográficas de segurança, na qual a segurançã de uma área pode ser adaptada a níveis mais elevados ou mais baixos dependendo da reputação dos veículos. Além, uma reputação complexa é examinada, na qual a reputação de um veículo está vinculada a varios fatores do comportamento. Uma outra área que é explorada é a avaliação de desempenho do ERPP o qual é conduzida através de simulações em um cenário urbano, com base na aplicação de encaminamento oportunista de mensagens. Os resultados mostraram a eficácia do ERPP em termos de avaliar o comportamento dos veículos e tomar medidas contra os veículos mal comportados. Utilizamos SUMO para simular o modelo de mobilidade; OMNET++ e Veins suportaram o modelo de red; and Crypto++ foi usado para implementar as funções criptográficas de curvas elípticas de assinatura e verificação de mensagens como recomendam os padrões de segurança. Finalmente, empregamos uma estratégia de mudança de pseudônimo na qual a reputação é discretizada em dois níveis de reputação. A estratégia foi implementada em um cenário de simulação de tráfego realista e foi comparada com as estratégias nomeadas de estado e síncrona mediante simulações. Os resultados mostraram que o número de pseudônimos utilizados em nossa estratégia é menor que os esquemas mencionados, e mantém a taxa de sucesso de mudança de pseudônimo alcançada pela estratégia síncrona.

Palavras-chave: Rede móvel, Rede veicular, Segurança, Sistema de Reputação, Gerenciamento de Confiança. 

Figure 1 - Intelligent Transport System (ITS) . . . . . . . . . . . . . . . . 32

Figure 2 - VANET communication domains . . . . . . . . . . . . . 32

Figure 3 - V2X communications domain . . . . . . . . . . . . 34

Figure 4 - DSRC spectrum band and channels allocation in the US. . . . . . . . . 38

Figure 5 - WAVE Architecture . . . . . . . . . . . . . . . 39

Figure 6 - Research Methodology . . . . . . . . . . . . . . . . . 49

Figure 7 - Simulation Tools . . . . . . . . . . . . . . . 51

Figure 8 - First three layers of a possible functional ontology of PPRS . . . . . . . 59

Figure 9 - Main object properties of PPRS . . . . . . . . . . . . 60

Figure 10 - Organisations and agents in PPRS . . . . . . . . . . . . . 61

Figure 11 - Complex representation of the reputation of a vehicle . . . . . . . . . 63

Figure 12 - General format of Data Message, DM . . . . . . . . . . . . . . 67

Figure 13 - Specific format of Data Message, DM . . . . . . . . . . . . . . 67

Figure 14 - Network model. . . . . . . . . . . . . . . . . . . . 69

Figure 15 - Finite state machine of the PPRS in the vehicle. . . . . . . . . . . 72

Figure 16 - Finite state machine of the PPRS in the Reputation Server. . . . . . . . . . . 74

Figure 17 - Registration process of a new vehicle in PPRS . . . . . . . . . . . 75

Figure 18 - Process of identity mapping anonymous to real. . . . . . . . . . . . 76

Figure 19 - Requesting the Reputation Certificate. . . . . . . . . . . . . . . 76

Figure 20 - Cryptographic functions for sending and receiving of messages. . . . . . . 78

Figure $21-$ Neighbouring discovery. . . . . . . . . . . . . . . . . . . 85

Figure 22 - Vehicle's reputation . . . . . . . . . . . . . . . 86

Figure 23 - Motivational scenario: sending and forwarding of messages in VANETs. . . 87

Figure 24 - Grid scenario for simulations. . . . . . . . . . . . . . . . . 89

Figure 25 - Percentage of fake messages varying the operation mode of the system. . . . 93

Figure 26 - Total results of reputation updates . . . . . . . . . . . . . . . . 94

Figure 27 - Results of $A R$ varying $M V$ with $\alpha=0.2, \delta_{k 1}=0.2$ and $1-\delta_{k 1}=0.8 \quad \ldots \ldots 4$

Figure 28 - Results of $A R$ varying $M V$ with $\alpha=0.2, \delta_{k 1}=0.5$ and $1-\delta_{k 1}=0.5 \quad \ldots$. . . 95

Figure 29 - Average reputation for the vehicles varying $\delta_{k 1}$ and $\delta_{k 2}=1-\delta_{k 1}$. . . . . . . 97

Figure 30 - Average reputation for the scenarios with $12.5 \%$ vehicles generating fake messages varying $\delta_{k 1}$ and $\delta_{k 2}=1-\delta_{k 1} \ldots \ldots \ldots$. . . . . . . . 97

Figure 31 - Public Key Infrastructure for VANETs . . . . . . . . . . . . . . . 100

Figure 32 - Pseudonyms being changed on the wrong occasion . . . . . . . . . . . 102 
Figure 33 - Pseudonym changing algorithm . . . . . . . . . . . . . . 106

Figure 34 - Selected views of the "joined" scenario from iTETRIS . . . . . . . . . 107

Figure 35 - Number of vehicles in the Bologna scenario. . . . . . . . . . . . . 107

Figure 36 - Comparison among strategies on the total number of used pseudonyms . . 110

Figure 37 - Comparison among strategies on the total number of successfully changed

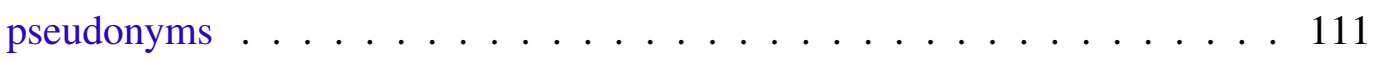

Figure 38 - Average time for pseudonym changing . . . . . . . . . . . 112

Figure 39 - Successful rate of changed pseudonyms . . . . . . . . . . . 112 
Table 1 - Security requirements versus VANET applications . . . . . . . . . . . 41

Table 2 - Overview of attacks in VANETs . . . . . . . . . . . . . . . 44

Table 3 - Summary of literature review. . . . . . . . . . . . . 56

Table 4 - Common symbols used for security and privacy . . . . . . . . . . . 64

Table 5 - Common symbols used for the reputation system . . . . . . . . . . 65

Table 6 - Primitives used in the FSM of the PPRS in vehicle . . . . . . . . . 71

Table 7 - Primitives used in the FSM of the PPRS in the server . . . . . . . . . 73

Table 8 - ECDSA schemes for signing and verifying of messages . . . . . . . . 77

Table 9 - Average cryptographic operation delay for the ECDSA (ms) . . . . . . . 77

Table 10 - SUMO configuration parameters . . . . . . . . . . . . . 88

Table 11 - Veins configuration parameters . . . . . . . . . . . . . . . 89

Table 12 - Planning of experiments to evaluate the impact of to change the operation mode in a geographical area . . . . . . . . . . . . . . . . 90 90

Table 13 - Planning of experiments to evaluate the impact of the weight $\delta_{k 1} \ldots \ldots$. . . 90

Table 14 - Results of Average Reputation under $\delta_{k 1}=0.2$ and $\delta_{k 2}=0.8 \ldots \ldots$. . . . . . 98

Table 15 - Results of Average Reputation under $\delta_{k 1}=0.5$ and $\delta_{k 2}=0.5 \ldots \ldots$. . . . . . 98

Table 16 - Comparing pseudonym refill strategies . . . . . . . . . . . . . . . 101

Table 17 - Mobility, scenario, and network parameters . . . . . . . . . . . . 108

Table 18 - Planning of experiments to evaluate the impact of the strategies of pseudonyms

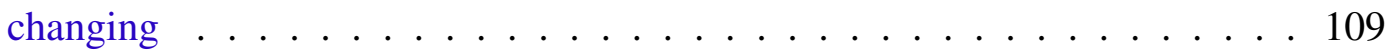





\section{LIST OF ABBREVIATIONS AND ACRONYMS}

\begin{tabular}{|c|c|}
\hline $\mathrm{ACM}$ & Area Condition Message \\
\hline ASN.1 & Abstract Syntax Notation One \\
\hline ASTM & American Society for Testing and Materials \\
\hline AU & Application Unit \\
\hline BBS & Boneh-Boyen-Shacham \\
\hline BSS & Basic Service Set \\
\hline $\mathrm{CA}$ & Certificate Authority \\
\hline $\mathrm{CC}$ & Check Code \\
\hline $\mathrm{CCH}$ & Control CHannel \\
\hline $\mathrm{CPU}$ & Central Processing Unit \\
\hline CRL & Certificate Revocation List \\
\hline DDoS & Distributed Denial of Service \\
\hline DLin & Decision Linear \\
\hline $\mathrm{DM}$ & Data Message \\
\hline DoS & Denial of Service \\
\hline DSRC & Dedicated Short Range Communication \\
\hline ECC & Elliptic Curve Cryptography \\
\hline ECDSA & Elliptic Curve Digital Signature Algorithm \\
\hline EDR & Event Data Recorder \\
\hline ELP & Electronic Licence Plate \\
\hline ETSI & European Telecommunication Standard Institute \\
\hline $\mathrm{EV}$ & Evaluated Vehicle \\
\hline FCC & Federal Communications Commission \\
\hline FM & Feedback Message \\
\hline FSM & Finite State Machine \\
\hline $\mathrm{FV}$ & Forwarder Vehicle \\
\hline GPS & Global Positioning System \\
\hline GUI & Graphical User Interface \\
\hline $\mathrm{HM}$ & Hello Message \\
\hline HRM & Hello Response Message \\
\hline I2I & Infrastructure to Infrastructure \\
\hline
\end{tabular}




\begin{tabular}{|c|c|}
\hline IDE & Integrated Development Environment \\
\hline ITS & Intelligent Transport System \\
\hline LBS & Location-Based Service \\
\hline LTE & Long Term Evolution \\
\hline MAC & Medium Access Control \\
\hline MANETs & Mobile Ad hoc NETworks \\
\hline MLME & MAC Layer Management Entity \\
\hline NIST & National Institute of Standards and Technology \\
\hline OBUs & On-Board Units \\
\hline OFDM & Orthogonal Frequency Division Multiplexing \\
\hline OMNET++ & Objective Modular NEtwork Testbed in C++ \\
\hline ON & Observer Node \\
\hline OSI & Open Systems Interconnection \\
\hline OV & Observer Vehicle \\
\hline PHYs & PHYsical \\
\hline PKI & Public Key Infrastructure \\
\hline PLME & Physical Layer Management Entity \\
\hline PPRS & Privacy-Preserving Reputation Scheme \\
\hline RA & Reputation Authority \\
\hline $\mathrm{RC}$ & Reputation Certificate \\
\hline $\mathrm{RM}$ & Resource Manager \\
\hline RQM & Reputation-Query Message \\
\hline RRM & Reputation-Response Message \\
\hline $\mathrm{RS}$ & Reputation Server \\
\hline RSUs & Roadside Units \\
\hline $\mathrm{SCH}$ & Service CHannels \\
\hline $\mathrm{SDH}$ & Strong Diffie Hellman \\
\hline SHA & Secure Hash Algorithm \\
\hline SIM & SIgnaling Message \\
\hline SNR & Signal-to-Noise Ratio \\
\hline SUMO & Simulation of Urban MObility \\
\hline TCP & Transmission Control Protocol \\
\hline TPD & Tamper-Proof Device \\
\hline TPM & Trusted Platform Module \\
\hline TRA & Transit Regulatory Authority \\
\hline TTP & Trusted Third Parties \\
\hline UDP & User Datagram Protocol \\
\hline
\end{tabular}


V2I Vehicle to Infrastructure

V2P Vehicle-to-Pedestrian

V2V Vehicle to Vehicle

V2X Vehicular to Everything

Veins Vehicular environment in network simulation

WAVE Wireless Access in Vehicular Environment

WSMP Wave Short Message Protocol 

$S C K_{v}^{+}$— Public key of the Security Certificate

$S C K_{v}^{-}$— Private key of the Security Certificate

$P_{v}$ - Pseudonym of a vehicle

$P K_{v}^{+}$— Pseudonym's public key

$P K_{v}^{-}$— Pseudonym's private key

$P I_{v}$ - Pseudo-Identity of a pseudonym

$e d-$ Emission date

$\mathrm{CAK}^{+}$— Public key of the Certificate Authority

$C A K^{-}$— Private key of the Certificate Authority

$A \mid B$ - Concatenation of data

\{\} - Digital signature

$\theta_{1}-$ Signature of the pseudonym

$\sigma_{1}$ - Signature of the data message

$\mathrm{RSK}^{+}$— Public key of the Reputation Server

$\mathrm{RSK}^{-}$— Private key of the Reputation Server

$R C_{v}$ - Reputation Certificate of a vehicle

$R C I$ - Identification of the Reputation Certificate

$\operatorname{Rep}_{v}$ - Reputation score of a vehicle

$K_{i} R e p-$ Reputation of the behavioural factor $i$

$\theta_{2}$ - Signature of the Reputation Certificate

$C C$ - Check Code

$\sigma_{2}-$ Signature of the feedback message

area - It defines friendly or unfriendly area

$\alpha-$ Factor of weight for the last rating

$\delta$ - Factor of weight for a behavioural factor 
PT — Punishing Threshold

$C T$ - Changing Threshold

list — List of intermediate vehicles

DMI — Data Message Identification

rating - Qualification of a feedback

rc_time — Time for updating the Reputation Certificate

secret_code - Code shared between the server and the vehicle 


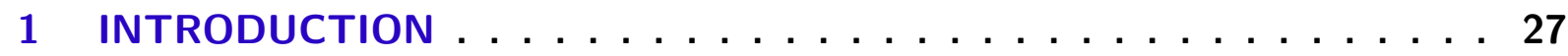

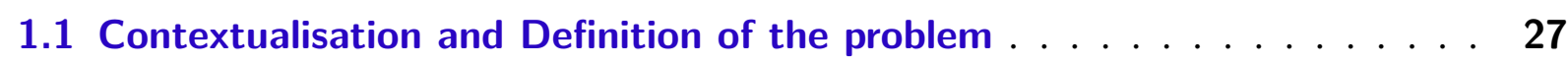

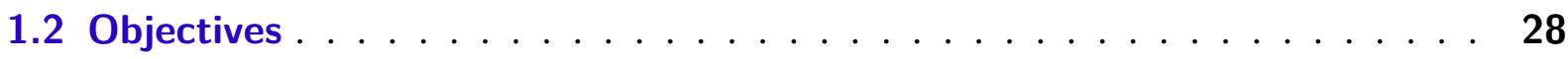

1.3 Contributions . . . . . . . . . . . . . . . . . . 29

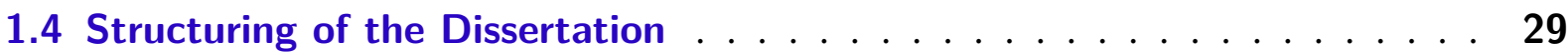

2 FUNDAMENTAL CONCEPTS . . . . . . . . . . . . . . 31

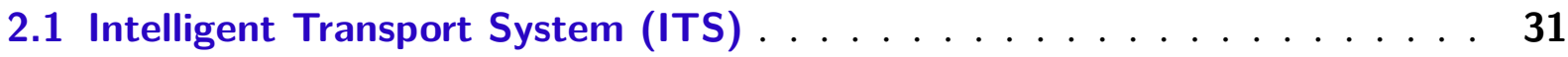

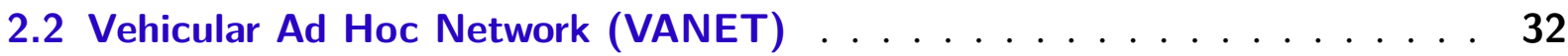

2.2.1 In-Vehicle Communication Domain . . . . . . . . . . . . . 33

2.2.2 Vehicular to Anything (V2X) Communication Domain . . . . . . 33

2.2.3 Infrastructure Domain . . . . . . . . . . . . . . . . . . . . . 34

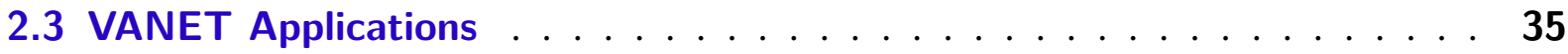

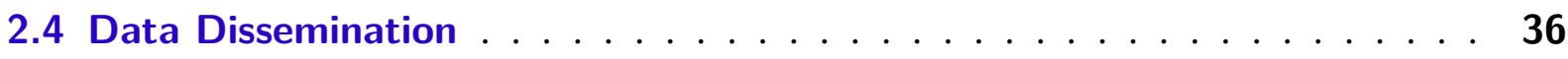

2.5 VANETs communication standards . . . . . . . . . . . . . 37

2.5.1 Dedicated Short Range Communication (DSRC) . . . . . . . . 37

2.5.2 Wireless Access in Vehicular Environments (WAVE) . . . . . . 38

2.5 .3 Standard IEEE $802.11 \mathrm{p} \ldots \ldots \ldots \ldots \ldots \ldots$

2.5.4 Standard IEEE 1609 WAVE . . . . . . . . . . . . . . . . 39

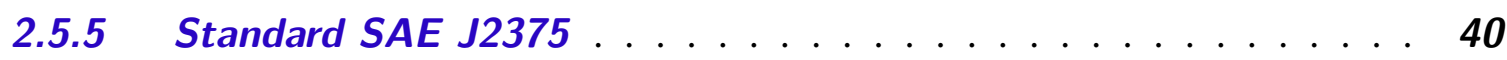

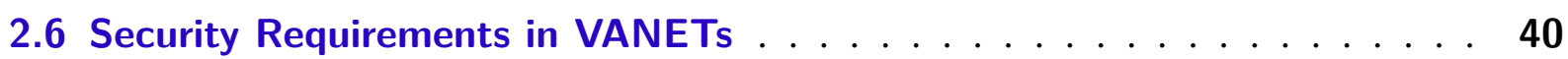

2.6.1 Authorisation. . . . . . . . . . . . . . . . 41

2.6.2 Identification . . . . . . . . . . . . . . . . . 41

2.6.3 Authentication . . . . . . . . . . . . . . . 42

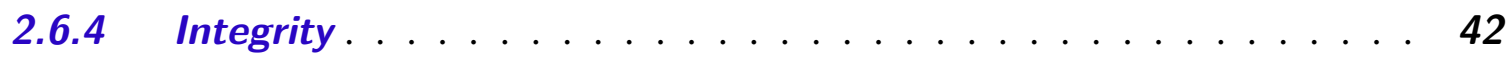

2.6.5 Data Confidentiality . . . . . . . . . . . . . . . . . 42

2.6 .6 Availability . . . . . . . . . . . . . . . . . . 42

2.6.7 Non-repudiation . . . . . . . . . . . . . . . . . . . . 42

2.6.8 Privacy, unlinkability and untraceability . . . . . . . . . . . 43

2.6.9 Trust management . . . . . . . . . . . . . . . . . 43

2.7 Classification of Attacks _. . . . . . . . . . . . . . . . . . . . 43

2.7.1 Attacks on Authentication/Identification . . . . . . . . . 44

2.7.2 Attacks on Integrity/Data Trust . . . . . . . . . . . . 45 
2.7.3 Attacks on Confidentiality . . . . . . . . . . . . . . . . 45

2.7.4 Attacks on Availability . . . . . . . . . . . . . . . 46

2.7.5 Attacks on Privacy . . . . . . . . . . . . . . . . . . 47

2.7.6 Attacks on Trust Management . . . . . . . . . . . . . 47

2.8 Public Key Infrastructure $(\mathrm{PKI}) \ldots \ldots \ldots$

2.9 Research Methodology . . . . . . . . . . . . . . . . . . . . 49

2.9.1 Simulation Tools . . . . . . . . . . . . . . . . 50

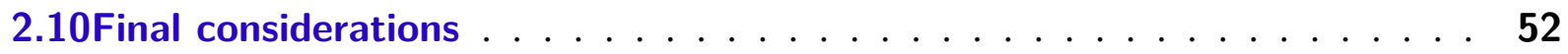

3 A PRIVACY-PRESERVING REPUTATION SCHEME . . . . . . . . . 53

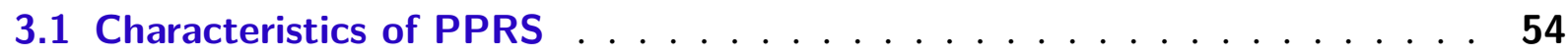

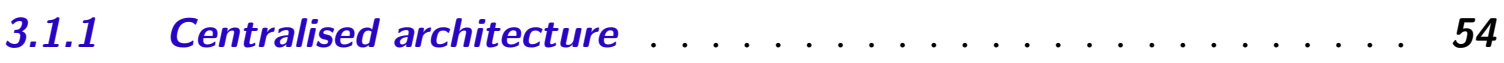

3.1.2 Properties of PPRS . . . . . . . . . . . . . . . . . . . . 54

3.2 Related Work . . . . . . . . . . . . . . . . . . . . . 55

3.2.1 Centralised reputation systems . . . . . . . . . . . . . . 56

3.2.2 Distributed reputation systems . . . . . . . . . . . . . 57

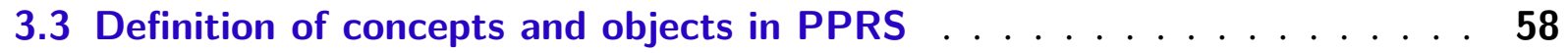

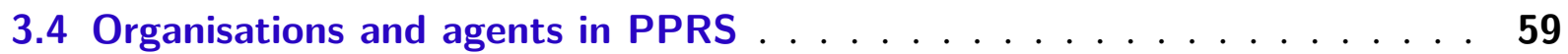

3.5 Entities and documents in PPRS . . . . . . . . . . . . . . 62

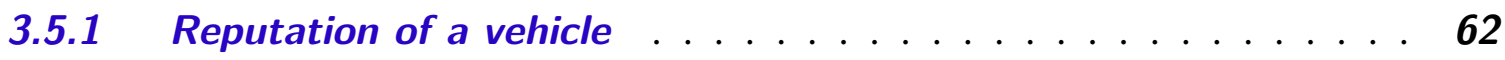

3.5.2 Behavioural factors of the complex reputation . . . . . . . . . 62

3.5.3 Geographical areas of security . . . . . . . . . . . . . 63

3.5 .4 Security Certificate $(S C) \ldots \ldots \ldots \ldots \ldots \ldots$

3.5 .5 Pseudonyms . . . . . . . . . . . . . . . . . . . . . 64

3.5.6 Reputation Certificate (RC) . . . . . . . . . . . . . . 65

3.5.7 Feedback . . . . . . . . . . . . . . . . . . . . 66

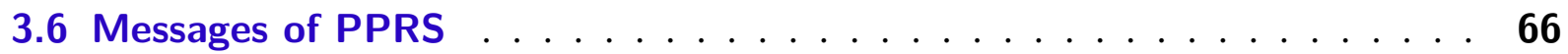

3.7 Network model . . . . . . . . . . . . . . . . . . . . . . . . . 69

3.8 Operation of PPRS in the vehicle $\ldots \ldots \ldots \ldots \ldots \ldots$

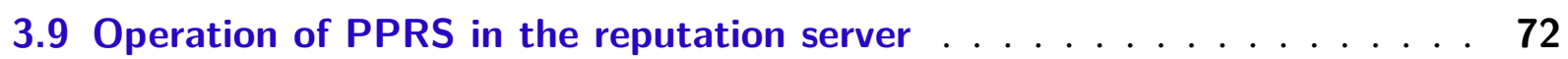

3.10 PPRS Phases . . . . . . . . . . . . . . . . . 73

3.11 Performance analysis . . . . . . . . . . . . . . . . 77

3.11 .1 Processing . . . . . . . . . . . . . . . 77

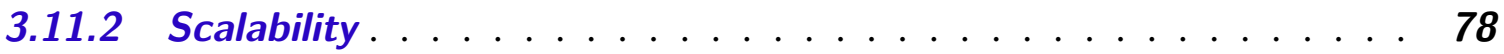

3.11 .3 Communications. . . . . . . . . . . . . . . . 78

3.11.4 Analysis of Robustness . . . . . . . . . . . . . . . 79

3.11.5 Overall operational forecasted costs . . . . . . . . . . . . 81

3.12 Final considerations . . . . . . . . . . . . . . . . . . . 81 
4 AN EVALUATION OF REPUTATION WITH REGARD TO THE OPPORTUNISTIC FORWARDING OF MESSAGES . . . . . . . . . . . . . 83

4.1 An opportunistic application for forwarding messages . . . . . . . . . . 83

4.1.1 The forwarding protocol . . . . . . . . . . . 85

4.1.2 Reputation of the vehicle . . . . . . . . . . . . . 85

4.1.3 Condition of the Geographical area . . . . . . . . . . . . . . 86

4.2 Motivational scenario . . . . . . . . . . . . . . . . . . 87

4.3 Simulation Setup . . . . . . . . . . . . . . . . . . . 88

4.3.1 Simulation Parameters . . . . . . . . . . . . . . . 88

4.3.2 Planning the experiments ................. 89

4.4 Results and discussions . . . . . . . . . . . . . . . . . . . 92

4.4.1 Evaluation of fake messages percentage, FMP . . . . . . . . . 92

4.4.2 Average Reputation (AR) in a scenario with low weight assigned for the generation of messages . . . . . . . . . . . . . . . . . 93

4.4.3 Average Reputation (AR) in a scenario with medium weight assigned for the generation of messages . . . . . . . . . . . . . . . 95

4.4.4 Effects of the weight for the generation of messages on the Average Reputation $(A R) \ldots \ldots$. . . . . . . . . . . 96

4.5 Final considerations . . . . . . . . . . . . . . . . . . . . 97

5 A PSEUDONYM CHANGING STRATEGY BASED ON REPUTATION LEVELS . . . . . . . . . . . . . . . . . . . . . 99

5.1 Definitions . . . . . . . . . . . . . . . . . . 100

5.1 .1 Privacy ....................... 100

5.1 .2 Conditional privacy . . . . . . . . . . . . . . . . 100

5.1.3 Pseudonyms . . . . . . . . . . . . . . . . . 101

5.1.4 Pseudonym certificate revocation . . . . . . . . . . . . . 101

5.1.5 Traceability problem .................. 101

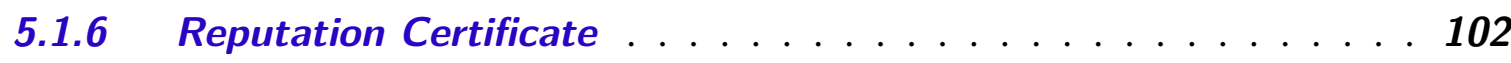

5.1.7 Anonymity . . . . . . . . . . . . . . . . . . 102

5.1.8 Reputation Level . . . . . . . . . . . . . . . . . . . 103

5.1 .9 Information about vehicular status . . . . . . . . . . . 103

5.2 Pseudonym Changing Strategies . . . . . . . . . . . . . . . . 103

5.3 Improving the synchronous change strategy using the Reputation Level (RL)105

5.4 Simulation setup . . . . . . . . . . . . . . . . . 105

5.4 .1 Simulation scenario . . . . . . . . . . . . . 106

5.4.2 Simulation parameters ................... 106

5.4.3 Planning the experiments . . . . . . . . . . . . . . . 107

5.5 Results and discussion . . . . . . . . . . . . . . . . . 110

5.5.1 Effect of the strategies on the number of used pseudonyms . . . 110 
5.5.2 Effect of the strategies on the number of successfully changed pseudonyms . . . . . . . . . . . . . . . . . 110

5.5.3 Effect of the strategies on the average time for pseudonym changes111

5.5.4 Effect of the strategies on the successful rate of changing pseudonyms112

5.6 Final considerations . . . . . . . . . . . . . . . . . 113

6 CONCLUSIONS AND FUTURE WORK . . . . . . . . . . . . . 115

6.1 Conclusion . . . . . . . . . . . . . . . . . . . 115

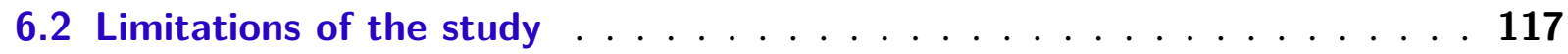

6.3 Future work . . . . . . . . . . . . . . . . . . 117

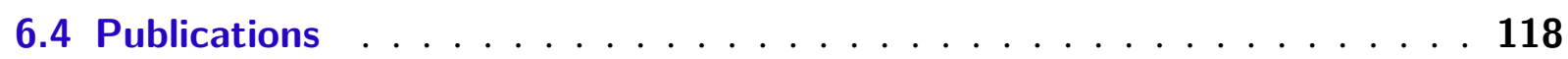

BIBLIOGRAPHY . . . . . . . . . . . . . . . . . 121 
CHAPTER

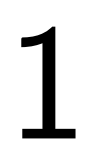

INTRODUCTION

\subsection{Contextualisation and Definition of the problem}

Vehicular Ad Hoc NETworks (VANETs) are a subclass of Mobile Ad hoc NETworks (MANETs) and are playing a key role in the Intelligent Transport System (ITS). In VANETs, vehicles are mobile nodes which form a dynamic network topology. Generally speaking, two different types of applications are envisioned for VANETs - safety and non-safety. The purpose of safety applications is to ensure that people's lives and public property are secure. Non-safety applications provide comfort and entertainment to travellers. The main components of VANETs include On-Board Units (OBUs) and Roadside Units (RSUs). The OBUs are fitted inside vehicles while the RSUs are deployed alongside the roads. Communication between vehicles is called Vehicle to Vehicle (V2V) and between vehicles and the RSU is called Vehicle to Infrastructure (V2I). Sometimes the term V2X is used to refer to all types of vehicular communications.

As VANETs are becoming a key component of ITS, the deployment of safety and nonsafety applications will be compromised unless there are proper security and privacy mechanisms. In this context, the IEEE 1609.2 (IEEE, 2013) and ETSI TS ITS 102-941 (ETSI, 2012a) security standards recommend the use of digital signature algorithms to ensure authenticity, integrity and non-repudiation in the exchange of sensitive messages. Privacy in VANETs is achieved through two properties - unlinkability and untraceability; unlinkability means that it should be impossible for an unauthorised entity to link the identity of the vehicle with that of its driver; and untraceability states that it should not be possible to trace the movements of the vehicle. The vehicles will be given pseudonyms issued by a Certificate Authority to provide both security and privacy, instead of long-term certificates that just guarantee security. Pseudonyms are short-term public key certificates that do not contain information that can reveal the identity of the driver. A pseudonym changing strategy must be adopted for the vehicles to prevent them from being tracked, since messages signed under the same pseudonym could be linked to each other. The vehicles will store the public key of the Certificate Authority to validate the pseudonyms, without 
having to access the infrastructure.

In the future, VANET applications will depend on cooperation between the nodes and sending of reliable messages for achieving their goals. For example, the users of a traffic application will make decisions based on the messages about the road conditions transmitted by part of the vehicles; fake messages will lead to take wrong actions. Similarly, routing protocols for VANETs are based on the assumption that all the nodes cooperate in the routing process. However, cooperation cannot always be guaranteed, as selfish nodes not cooperate without earning some points or being given incentives. The existence of selfish and misbehaving nodes in VANETs is a serious problem, as it impairs the performance of the system. VANETs can only improve traffic safety, if the messages sent by vehicles are trustworthy (WU; DOMINGOFERRER; GONZÁLEZ-NICOLÁS, 2010).

The traditional security mechanisms do not look after that authorised vehicles send bogus or fake messages that in the worst case put at risk the people's lives. In view of this, trust management is another important component of security services in VANETs, which provides a unified strategy for establishing a relationship between the nodes and assisting in recording the behaviour of the vehicles. Managing the evolving pattern of vehicular behaviour must take into account that the communication between them is anonymous, and this is a challenging task (CHAURASIA; VERMA, 2013). Moreover, since the pseudonyms of vehicles are constantly being changed to prevent tracking, providing a balanced solution that meets the requirements of security, privacy, and trust management in VANETs, is also a complex matter.

\subsection{Objectives}

The purpose of this project is to create awareness of the need for anonymous communication and to provide privacy to vehicles, while enabling them to build trustworthy relationships which each other. With regard to this, the specific objectives are as follows:

- To model, design, implement and evaluate the performance of the Preserving-Privacy Reputation Scheme PPRS for VANETs;

- To establish trust in the VANETs through a centralised reputation system that is aimed at recording the behaviour of the vehicles. This involves rewarding the honest vehicles and punishing those that misbehave;

- To improve privacy by means of the unlinkability and untraceability properties in VANETs through a pseudonym-based mechanism and a pseudonyms changing strategy. 


\subsection{Contributions}

The first contribution made by this dissertation is an investigation of the PrivacyPreserving Reputation Scheme (PPRS) for trust management in VANETs, which has the following features:

- The scheme provides a dynamic reputation mechanism by introducing geographical areas of security in which the security of an area can be adapted to higher or lower levels depending on the reputation of the neighbouring vehicles that reflects the threat level that exists in the area;

- In our scheme, the reputation of a vehicle is not a single and abstract concept but rather a multifaceted concept, i.e., a complex feature. The vehicles receive feedback with regard to several behavioural factors, thus that their reputations can be inferred from an ontology.

The second contribution is the implementation of PPRS in an opportunistic network application for forwarding messages. The vehicles are given feedback on the behavioural factors involved in generating and forwarding messages. The scheme reflects the behaviour of both the honest vehicles, and the misbehaving vehicles that send fake messages.

The third contribution is the incorporation of the reputation level of the vehicles as a part of the information needed for making the decision about pseudonym changing, and in this way being able to show that our scheme does not jeopardise the existing mechanisms and optimises the use of pseudonyms in VANETs.

\subsection{Structuring of the Dissertation}

The rest of this dissertation is divided into the following chapters:

- Chapter 2 defines some basic concepts employed for VANETs: i) there is a description of the VANET communication domains, including In-Vehicle, V2X and the infrastructure; ii) there is an outline of the VANETs applications, the problems of the data dissemination in sparse or dense networks, as well as seeking solutions for our scheme; iii) it is related the most important standards for VANETs, together with the security requirements, including privacy and trust management among other factors; iv) an attempt is made to list the most serious attacks on VANETs; v) there is a review of some aspects of the Public Key Infrastructure (PKI); vi) the research methodology is detailed; vii) it exposes a summary of the final considerations of the chapter;

- Chapter 3 describes the Privacy-Preserving Reputation Scheme (PPRS) proposed in this project: i) there is an outline of the main features of the scheme; ii) it examines the related 
work on reputation system for VANETs; iii) there is a discussion of the main concepts and features that form the building blocks of a system using PPRS; iv) it provides a list of the organisations and agents involved in PPRS; v) there is a description of the entities and documents in PPRS; vi) there is a detailed examination of the messages, network model, operations of the PPRS both in the vehicle and in the server, and the different phases of PPRS; vii) it analyses the performance of the PPRS; and viii) it adds some final considerations of the chapter;

- Chapter 4 explains how the PPRS is implemented in an opportunistic network application for forwarding messages and its most significant results: i) there is a description of the application and the main concepts; ii) it sets out the motivational scenario; iii) it examines the simulation environment including the mobility and network parameters used for planning and conducting our experiments; iv) there is an analyses of the results of the simulation experiments which take the average reputation obtained by the vehicles; v) it exposes the final considerations of the chapter.

- Chapter 5 provides a detailed description of the pseudonym changing strategy based on reputation levels of the vehicles: i) it is defined the main aspects of our strategy, including the problems of linkability and traceability; ii) it abstracts the work related to pseudonym changing strategies; iii) it sets out a new method based on the Synchronous Change strategy including the Reputation Level of the vehicles; iv) there is a discussion of the simulation setup; v) it analyses the results of the simulation experiments by taking the number of pseudonyms changed as the main metric; and vi) there are some final considerations of the chapter;

- Chapter 6 summarises this work and concludes the dissertation. In a similar way, we provide a list of some of the limitations of this work. Finally, there are some directions for future work in the field. 
CHAPTER

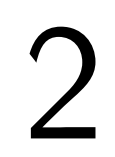

2

\section{FUNDAMENTAL CONCEPTS}

Vehicular Ad Hoc NETwork (VANET) is a key major of the communications in the Intelligent Transport System (ITS). The VANET has been studied mainly for safety applications with the goal of reducing the number of accidents on the roads. The VANET has also been planned for non-safety applications which provide comfort, assistance and support in the travels. The VANET receive a great deal of attention from different institutions and organisations because its importance to the ITS, more specifically to public safety. This chapter describes the main concepts on VANETs, related to communication domains, applications, data dissemination, standards, security requirements, attacks, security architecture and research methodology used in our proposal. Finally, the chapter concludes with some final considerations.

\subsection{Intelligent Transport System (ITS)}

ITS is a generic term for the integrated application of communications, control and information processing technologies of the transport system (ETSI, 2012b). The interest in ITS comes from the problems caused by traffic congestion and a synergy of new information technology for simulation, real-time control, and communications networks. Traffic congestion has been increasing worldwide as a result of urbanisation and population growth. Congestion reduces the efficiency of the transport infrastructure and increases the travel time, air pollution, and fuel consumption. The objectives of ITS are saving people's lives, minimising injuries caused due to accidents, reducing the travel time by avoiding traffic jams, keeping the environment green by means of the reducing of emission of $\mathrm{CO} 2$ and providing entertainment to the passengers and drivers. The key function of the ITS is to help the decision-making by transport network controllers and other users, and thus improving the operation of the entire transport system. Although, the main focus of the ITS is on road transport, it also includes rail, marine and air transport. A high level view of the ITS is shown in Figure 1. 
Figure 1 - Intelligent Transport System (ITS)

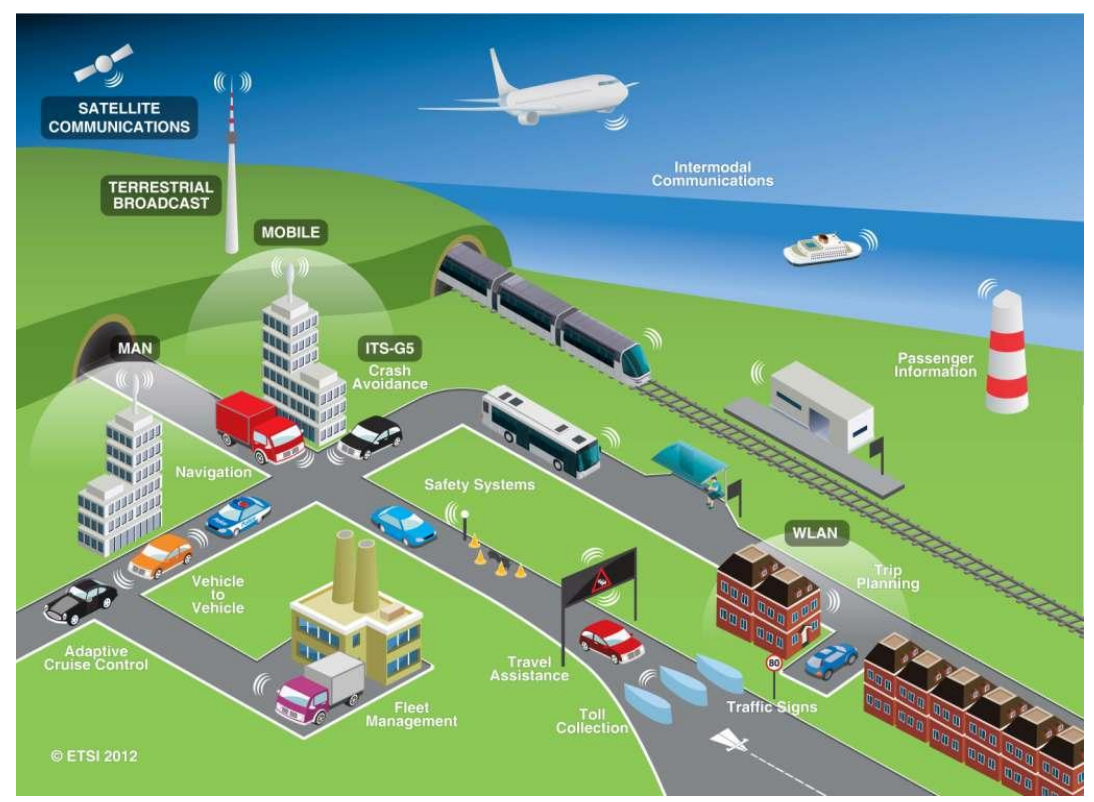

Source: ETSI (2012b).

\subsection{Vehicular Ad Hoc Network (VANET)}

Mobile Ad Hoc NETwork (MANET) is an autonomous system made up of mobile stations interconnected by wireless connections without the management of a centralised infrastructure. VANET is a subclass of MANET in which the vehicles act as moving nodes forming a dynamic network topology and can play the role of a router to relay data (ULLAH et al., 2013). The nodes travel at much higher speeds compared to the traditional MANET and can take predictable routes randomly. In the following subsections, the VANETs communication domains are detailed, as shown in Figure 2.

Figure 2 - VANET communication domains

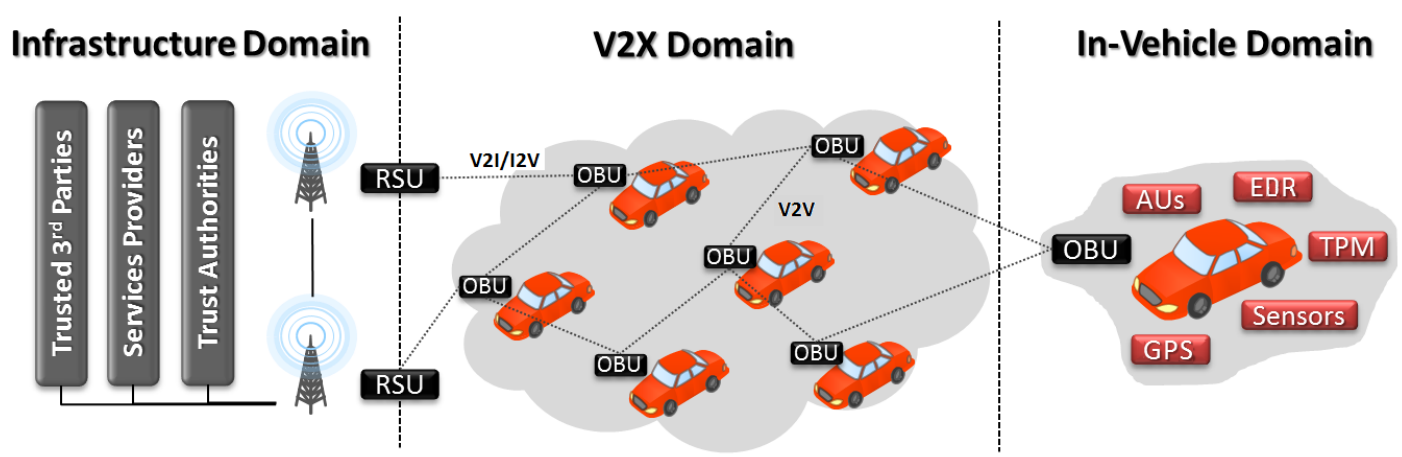

Source: Hamida, Noura and Znaidi (2015). 


\subsubsection{In-Vehicle Communication Domain}

The in-vehicle domain consists of the following elements included in a smart vehicle: the Event Data Recorder (EDR), wireless-enabled On-Board Unit (OBU), Tamper-Proof Device (TPD) or Trusted Platform Module (TPM), Application Unit (AU), Global Positioning System (GPS) device, Graphical User Interface (GUI), and set of sensors. These modules collaborate in the exchange of messages, and form an in-vehicle network (it also known as the on-board network, see Figure 2).

The vehicle is able to communicate with other close ITS entities using the communication capabilities of the OBU (HAMIDA; NOURA; ZNAIDI, 2015). The AU is responsible for running one or multiple applications, which are offered by remote Service Providers (SPs). The vehicle uses the GPS unit to obtain its accurate location information. The EDR is responsible for recording critical data of the vehicle (such as position, speed, time, etc.) during emergency events, similar to the black box of an air-plane. These data will help in reconstruction of accidents and accountability. If some investigation is carried out, these messages can be extracted and used as evidence. The EDR has been installed in vehicles such as trucks (HUBAUX; CAPKUN; LUO, 2004).

The TPD functions as a Central Processing Unit (CPU) of general purpose which is able to process and provide hardware protection, so that it cannot be easily penetrated by anyone who is not authorised for do it. It stores digital certificates and cryptographic keys, and performs the cryptographic operations of signing and checking of sensitive messages. However, the TPD is of high cost for its mass deployment and could only be expected in high-end vehicles (DOMINGOFERRER; WU, 2009). An alternative option to TPD is TPM, which it is able to prevent different software attacks, but not to sophisticated hardware tampering (RAYA; HUBAUX, 2007). Such units are gaining wide usage in notebooks and cost only a few tens of dollars. The final definition of the security hardware will depend mainly on economic and technical factors.

\subsubsection{Vehicular to Anything (V2X) Communication Domain}

The kinds of V2X communications include: Vehicle-to-Vehicle (V2V) communications between neighbouring vehicles; Vehicle-to-Infrastructure (V2I) communications between vehicles and the infrastructure, and vice versa; and Vehicle-to-Pedestrian (V2P) communications between vehicles and the surrounding pedestrian. In Vehicular to Everything (V2X), X can also include i.e., motorcycle, pedestrian, home, etc.

The main communication components are the On Board Units (OBUs) into vehicles and the Roadside Units (RSUs) deployed along the roads. The OBU has read-write memory, processor, Graphical User Interface (GUI) to exchange information with the drivers, and wireless interface which has an external radio antenna with Dedicated Short Range Communication (DSRC) technology. The wireless interface provides short-range wireless communications with 
Figure 3 - V2X communications domain

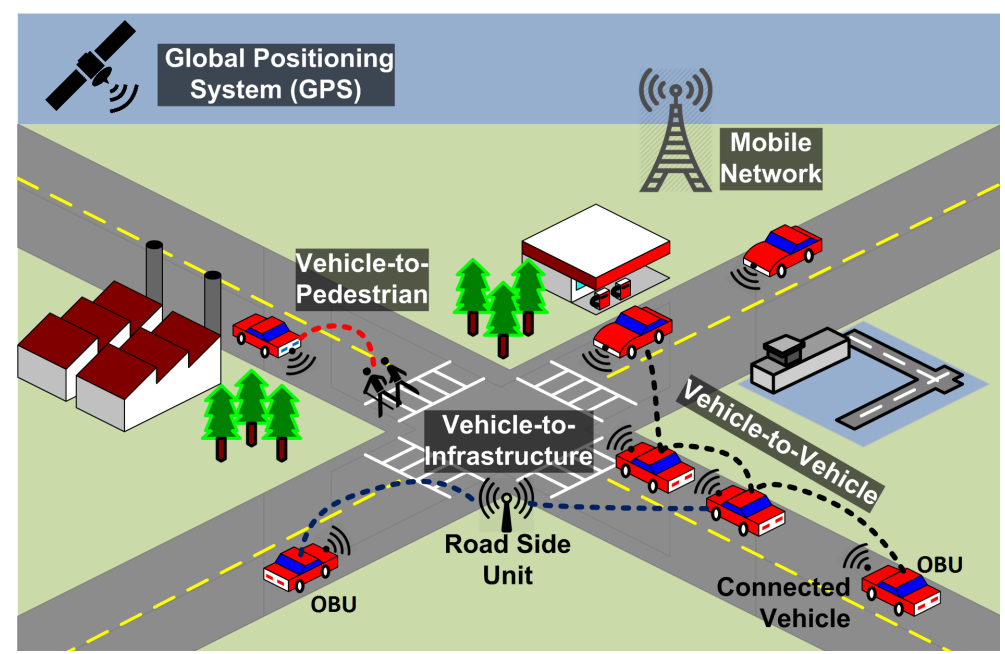

Source: Hamida, Noura and Znaidi (2015).

others OBUs as well as with RSUs. The OBU broadcasts periodic messages called beacons, which contains vehicle information about its position, time, direction, and speed, etc. As shown in Figure 3, the vehicles collect and process information that later it is sent to the close vehicles or RSUs by means of wireless communications.

The RSUs are stationary units installed at fixed locations alongside main roads, and other strategic locations (e.g., crossroads and parking lots). Similar to OBU, the RSU also has a read-write memory, processor, and wireless interface with DSRC technology. In addition, it also has a second wired or wireless interface, which is used for communications with other RSUs and entities in Internet. The RSU can be considered as a bridge between the V2X and the infrastructure. The RSUs can be under the control of government entities (e.g., vehicular authority) or a reliable third party broker.

\subsubsection{Infrastructure Domain}

The infrastructure domain includes the following entities which are permanently interconnected through Internet (see Figure 2):

i. Trusted Third Parties (TTP): they offer different services to the vehicles as credential management or time-stamping. Both manufacturers and the Certificate Authority are related to TTPs because the vehicles eventually need their services (e.g., for issuing electronic credentials);

- Manufacturers: they identify the vehicles as part of the manufacturing process. The long-term security certificate for the vehicle is generated at manufacturing time; however, it can be updated later by trusted authorities; 
- Certificate Authority (CA): it issues the security certificate and pseudonyms to the vehicles as part of the processes of security and privacy,

ii. Service Providers (SPs): they offer services that can be accessed through the VANET, e.g., Location-Based Service (LBS). The SPs provide applications to the vehicles and are responsible for managing software updates, billing and delivery of added-value services.

iii. Transit Regulatory Authority (TRA): it realises two tasks to know - vehicle registration and offence reporting. Every vehicle in an administrative region must be registered after manufacturing and receive its Electronic Licence Plate (ELP) by the TRA;

\subsection{VANET Applications}

VANET provides an environment for the deployment of numerous potential applications referred as safety and non-safety applications. In this section, the non-safety applications are presented in traffic optimisation, and infotainment and comfort applications as in (KOSCH et al., 2009).

Safety applications: these applications aim to reduce the probability of transit accidents and damage caused by them. The drivers are notified with messages about roads condition, closer vehicles and accidents occurrence. The common characteristic of this category is the relevance to life-critical situations in which the existence of a service may prevent life-endangering accidents. Hence, the security of this category is mandatory, since the proper operation of a safety application should be guaranteed, even in the presence of attackers (RAYA; HUBAUX, 2007). The critical latency (or end-to-end communication delay) also represents one important requirement which typically should not exceed og one hundred milliseconds. Safety applications require that the vehicles periodically (with a period between $100 \mathrm{~ms}$ and $500 \mathrm{~ms}$ ) broadcast beacons which contain their current state.

Traffic optimisation: the increasing number of vehicles causes more traffic delays, especially during the rush- hours. The VANET can reduce traffic delays by notifying drivers about the traffic, weather, road conditions, construction zones, main road or rail crossroads, emergency vehicle signal preemption, etc. Applications such as "driving assistance" will help the drivers to enjoy smooth and easy driving by avoiding possible conflicts. VANET will allow managing efficiently vehicles electronically by the transport administration authorities (DOMINGO-FERRER; WU, 2009). In general, these applications rely on the periodic broadcast of messages and/or unicast V2X communications, whose critical latency should typically not exceed one hundred milliseconds (HAMIDA; NOURA; ZNAIDI, 2015).

Infotainment and comfort applications: these applications aim at enhancing the driving experience by providing the drivers with various value-added services. These services are generally offered by trusted SPs, where the applications and services are downloaded and in- 
stalled on the AU of the vehicle. An application example is the remote vehicle diagnostic and maintenance of vehicles, in which the SP collects information from the in-vehicles sensors and sends notifications to the drivers regarding detected safety defects and/or to remind them about planned car maintenance. The vehicle will communicate with the data centre of the SP using V2I communications whose latency should typically not exceed five hundred milliseconds (HAMIDA; NOURA; ZNAIDI, 2015).

\subsection{Data Dissemination}

In the case of VANETs with numerous vehicles in an area, it is a big challenge to model trustworthiness between peers. This may introduce network congestion, since the vehicles are communicating on a shared channel, and there is overheading of messages which prevent vehicles from receiving data from the close vehicles (ZHANG, 2011). The disconnected or fragmented network problem is also a research challenge for developing reliable and efficient protocols in VANETs. Vehicles on freeways or urban areas are more likely to form highly dense networks during the rush- hours, while they are expected to experience frequent network fragmentation in sparsely populated rural freeways or late in the evening.

The concept of data dissemination is generally referred to as a process of spreading data or information over distributed wireless networks, and its approaches in VANET can be classified in flooding or relaying. In flooding, each vehicle broadcasts data to all the vehicles in the vicinity. This approach is good for delay sensitive applications and also for networks during low traffic conditions (TOMAR; CHAURASIA; TOMAR, 2010). However, this approach can cause a broadcast storm problem when the network density increases. It does not perform well in sparse networks, since the messages do not reach isolated network segments(MENEGUETTE et al., 2016). In relaying, a single relay node is selected between the neighbours as the next hop, this node will forward the data to the next hop and so on. The main advantage of this approach is that reduces the network congestion and is scalable to dense networks (TOMAR; CHAURASIA; TOMAR, 2010).

In our project, the data dissemination follows both approaches flooding and relaying. In the protocol for forwarding data messages (see Subsection 4.1.1), the first phase is based on broadcasting the Hello message to the neighbour vehicles of one hop, the second phase consists in to select the next hop among the vehicles that answering to Hello, and the third phase is based on the relaying type described above. This protocol does not lead to the broadcast storm problem in dense networks, but cannot perform well in a sparsely connected network. When the sender vehicle of a message is far away from the destination, the message has to go through multiple hops over long distance to reach the target. For mitigating this, the protocol also uses the store-carry-forward approach, increasing the chances of the message to reach the destination. An intermediate vehicle stores the message for a time period, until it selects another intermediate 
vehicle to continue the forwarding of the message.

The reputation server disseminates a state message from the infrastructure to the VANET. This dissemination can present problems in dense networks because it is based on broadcast communication, and can fail in sparse networks, avoiding that the vehicles update the security state. Nevertheless, some controls to mitigate these drawbacks were applied: i) vehicles that hear the transmission of a message that is already scheduled to forward cancel their transmissions to minimise the number of broadcast messages (VILLAS et al., 2012), ii) the vehicle holds the state message for a time period, and then forward it for a controlled number of times (KIM et $a l ., 2007)$, iii) a unique identifier is assigned for each message, thus the vehicles prevent the reception of duplicate messages. Most communications of the reputation system in our scheme occur between the vehicle and the RSU to exchange reputation and feedbacks information. Hence, a lot of communications in the RSU's area can cause collisions and reduce the system performance. Our scheme implements Elliptic Curve Cryptography (ECC) with much smaller public key sizes than the others digital signature algorithms, leading to significant performance advantages in the dissemination of messages. For future, there are two approaches to improve the V2I communications: a) to optimise the protocol 802.11p as in (CALAFATE et al., 2012) and b) to incorporate new technologies such as Long Term Evolution (LTE) (YANG et al., 2014).

\subsection{VANETs communication standards}

This section briefly surveys the most relevant standards to address different aspects of the ITS. These efforts encompass various multidisciplinary areas, including radio channel modelling, data link protocols, wireless communications, networking protocols, security, data privacy and localisation.

\subsubsection{Dedicated Short Range Communication (DSRC)}

The VANET standardisation process started after the allocation of the Dedicated Short Range Communication (DSRC) spectrum band in the United States in 1999. The Federal Communications Commission (FCC) assigned $75 \mathrm{MHz}$ of licensed spectrum, from $5.85 \mathrm{GHz}$ to $5.925 \mathrm{GHz}$ specifically for the use of vehicular communications.

As shown in Figure 4, the DSRC spectrum is structured into seven $10 \mathrm{MHz}$ channels. Channel 178 is the Control CHannel $(\mathrm{CCH})$, which is restricted to safety applications only. The channels 172 and 184 are reserved for special uses. The channels 174, 176, 180 and 182 are Service CHannels ( $\mathrm{SCH}$ ) and can be used for both safety and non-safety applications (GROUP et al., 2002). Similar bands have been allocated in Japan and Europe. The European Telecommunication Standard Institute (ETSI) allocated 70MHz for the ITS in the $5.9 \mathrm{GHz}$ band, within the frequency range from $5.850 \mathrm{GHz}$ to $5.925 \mathrm{GHz}$ (ETSI, 2005). In Japan, the $10 \mathrm{MHz}$ 
band from $715 \mathrm{MHz}$ to $725 \mathrm{MHz}$ was assigned to ITS using V2V communications (SAI et al., 2009).

Figure 4 - DSRC spectrum band and channels allocation in the US.

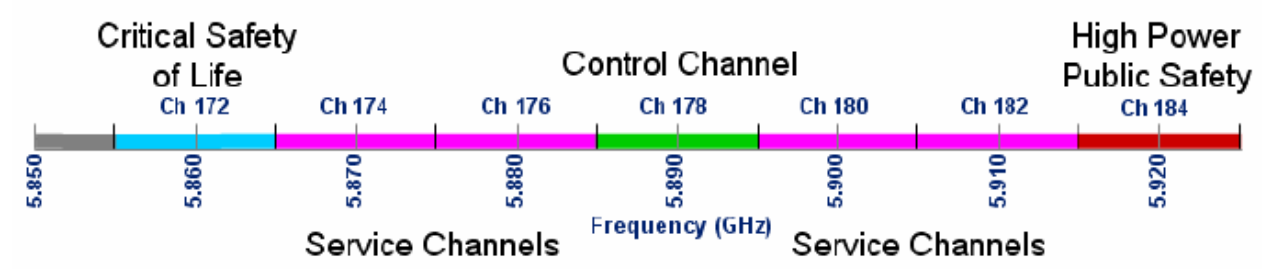

Source: Jiang and Delgrossi (2008).

\subsubsection{Wireless Access in Vehicular Environments (WAVE)}

The IEEE 802.11p and IEEE 1609.x standards are collectively called Wireless Access in Vehicular Environment (WAVE). They provide an architecture for the vehicular communications targeted for the use of safety and traffic optimisation applications, which follows a layered approach as the Open Systems Interconnection (OSI) model, see Figure 5. The architecture supports two protocol stacks, one for data transmission and another for management. Both stacks use the same physical and data-link layers of the OSI model, and differ in the network and transport layers. WAVE does not specify the session or presentation layers, however it introduces the blocks of security services and resource manager that do not fit within the OSI model (UZCÁTEGUI; SUCRE; ACOSTA-MARUM, 2009).

\subsubsection{Standard IEEE 802.11p}

The American Society for Testing and Materials (ASTM) modified the 802.11a standard to better match the vehicular environment. Based on this effort, the IEEE developed the standard named IEEE 802.11p (HARTENSTEIN; LABERTEAUX, 2008). The 802.11p standard defines the data transmission and management functions of the physical (PHY) layer and part of the data-link (Media Access Control - MAC) layer of WAVE architecture. The management functions are referred as Physical Layer Management Entity (PLME) and MAC Layer Management Entity (MLME), see Figure 5.

Physical layer (PHY): 802.11p made minimum changes to the IEEE 802.11 PHY layer, so that the WAVE devices can effectively communicate vehicles at high speed on main roads. It is based on an Orthogonal Frequency Division Multiplexing (OFDM) and uses channels of 10 $\mathrm{MHz}$ as opposed to the channels of $20 \mathrm{MHz}$ used by the IEEE $802.11 \mathrm{a}$. The data rate goes from 3 to $27 \mathrm{Mbps}$ for each channel, the lower rates are often preferred to obtain robust communication (HARTENSTEIN; LABERTEAUX, 2008). 
Figure 5 - WAVE Architecture.

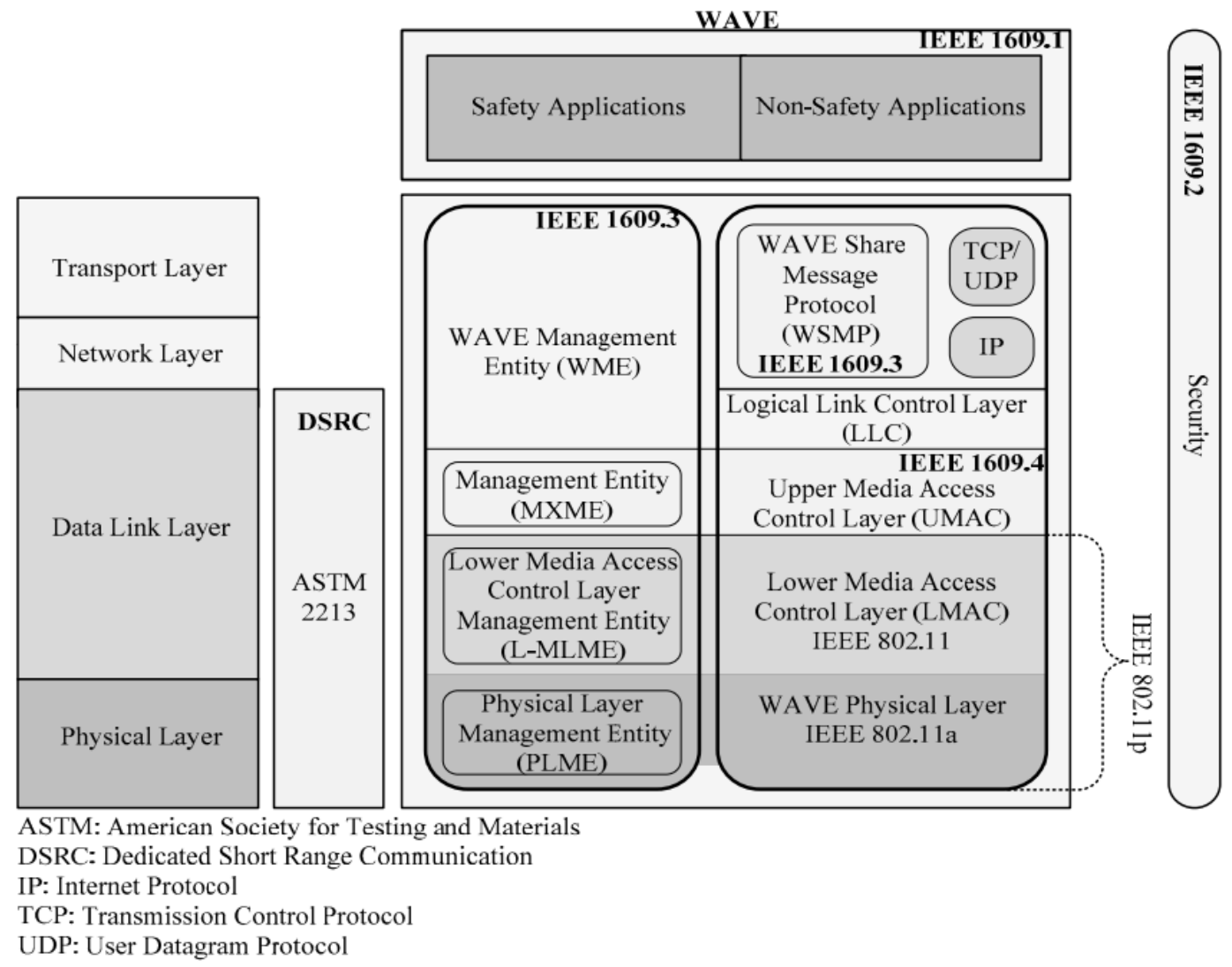

Source: Zeadally et al. (2012a).

MAC layer: the main contribution at the MAC level was to enable very efficient group communication setup without the overhead needed in the current IEEE 802.11 MAC layer (JIANG; DELGROSSI, 2008). The protocol facilitates to the stations to operate in a rapidly varying environment without having to join a Basic Service Set (BSS), as in the traditional IEEE 802.11 .

\subsubsection{Standard IEEE 1609 WAVE}

The IEEE 1609 standard deals the aspects of operation and management of the network and transport layers, as well as part of the data-link layer of the WAVE architecture. It also defines the resource management and security services blocks. The IEEE 1609 protocol suite consists of four standards:

i. IEEE 1609.4: this standard describes multichannel wireless radio operations, WAVE mode, the Medium Access Control (MAC) and PHYsical (PHYs) layers, including the operation of $\mathrm{CCH}$ and $\mathrm{SCH}$ interval timers, parameters for access priority, channel switching and routing, management services, and primitives for multichannel operations (IEEE 1609.4, 2006). 
ii. IEEE 1609.3: the networking protocol provides addressing and routing services within a vehicular environment. The IEEE 1609 framework has on the top of IEEE 802.11p two parallel protocol stacks: one for User Datagram Protocol (UDP) /Transmission Control Protocol (TCP) over IPv6, and another called Wave Short Message Protocol (WSMP). In WSMP can be specified low level parameters as data rate and transmitted power level (IEEE 1609.3, 2007).

iii. IEEE 1609.2: it specifies a range of security services for use in the WAVE environment. Mechanisms are provided to authenticate WAVE management messages that do not require anonymity, and to encrypt messages to a known recipient (IEEE 1609.2-2006, 2006). A revised version of the standard IEEE 1609.2-2006 is documented in (IEEE 1609.2-2013, 2013).

iv. IEEE 1609.1: this standard specifies the WAVE application called Resource Manager (RM), designed to allow applications at remote sites to communicate with the OBU of the vehicles through a RSU. The RM multiplexes the communications of multiple remote applications. The purpose of the communication is to conduct information necessary to implement the requirements of the remote WAVE applications (IEEE 1609.1, 2006).

\subsubsection{Standard SAE J2375}

Applications in vehicular networks suggest the adoption of a set of common messages to facilitate the interchange of information at the application layer. The standard SAE J2375 specifies a message set, its data frames and elements specifically for being used by applications intended to utilise the DSRC technology (SAE, 2009). The Abstract Syntax Notation One (ASN.1) specifies the format of these messages. The standard would help to solve the interoperability of the applications in VANETs through the implementation of messages in a pre-established format (HEDGES; PERRY, 2008). ETSI also constituted the standard called ETSI ITS-G5 (ETSI, 2009). Similar standardisation efforts were realised in Japan with the standard called ARIB STD-T75 (ARIB, 2001).

\subsection{Security Requirements in VANETs}

Security characteristics and requirements of VANETs differ quite significantly from traditional ad hoc networks. Highly dynamic environments of VANETs need an adapted form of security mechanisms. General requirements for all computational system are access control, confidentiality, integrity, availability, authentication and non-repudiation. In vehicular networks the privacy has been identified to be profoundly important (GERLACH, 2006), as well as the trust in the sender to make decisions effectively in the receiver based on the received information (YANG, 2013). 
As shown in Table 1, the below security requirements depend on the considered application type. For instance, traffic safety applications involve the broadcast of cooperative awareness messages. In this context, ensuring the confidentiality and access control of the messages is not required, since all the neighbouring vehicles should be able to receive and decode these safety messages, which contain public data. In contrast, the confidentiality and access control in applications of infotainment and comfort is optional because of that will depend on the nature of the data transmitted in a particular application. The VANET needs to guarantee the acceptable availability with the minimum delay for each type of application. Considering that privacy and trust management are necessaries for all the applications, this brings that integrity, authentication and non-repudiation of the sender are also fundamentals for the correct functioning of a reputation system. Due to the goal of the traffic safety applications of saving lives and reducing accidents, the authentication, integrity, availability, non repudiation and trust management are considered mandatory requirements.

Table 1 - Security requirements versus VANET applications

\begin{tabular}{l|l|l|l}
\hline Security requirement & Traffic safety & Traffic optimisation & Infotainment and comfort \\
\hline \hline Access control & - & - & optional \\
\hline Authentication & $\checkmark$ (sender) & $\checkmark$ (sender) & $\checkmark$ \\
\hline Integrity & $\checkmark$ (sender) & $\checkmark$ & $\checkmark$ \\
\hline Confidentiality & - & -- & optional \\
\hline Availability & $\checkmark$ (sender-rec) & $\checkmark$ & $\checkmark$ \\
\hline Non-repudiation & $\checkmark$ (sender) & $\checkmark$ (sender) & $\checkmark$ \\
\hline Privacy & $\checkmark$ & $\checkmark$ & $\checkmark$ \\
\hline Trust management & $\checkmark$ (sender) & $\checkmark$ & $\checkmark$ \\
\hline \hline
\end{tabular}

Source: Research data.

$\checkmark$ recommended $\checkmark$ mandatory - not required

\subsubsection{Authorisation}

Access control means to provide the user the ability to control access to other vehicles, infrastructure and applications through communication channels. Access control policies can be implemented in value-added services. By default, in safety and traffic optimisation applications the information is open to all other vehicles.

\subsubsection{Identification}

In VANETs, each vehicle requires a unique identification scheme. One of the common ways is the use of special plate licences called Electronic Licence Plate (ELP), issued by the Transit Regulatory Authority (HUBAUX; CAPKUN; LUO, 2004; RAYA; HUBAUX, 2005). This identity should be unique and cryptographically verifiable, attached to a public key certificate issued by the Certificate Authority to identify vehicles. 


\subsubsection{Authentication}

In secure VANETs, the information must come from legitimate vehicles, therefore an authentication mechanism is required. In some cases, only the sender needs to be authenticated, while in other situations, it is important to authenticate the receiver vehicle. There are two types of authentication: entity and attribute. In the entity authentication, the receiver vehicle checks the identity of the sender vehicle. The attribute authentication is used in group communications to prove that participants have the required attributes to become group members (FUENTES; GONZÁLEZ-TABLAS; RIBAGORDA, 2010).

\subsubsection{Integrity}

Message integrity ensures that the content of a message has not been altered during its transmission, which means that the data is truthful. Moreover, the integrity is able to resist destruction, unauthorised creation and alteration of data. False or modified data can produce potential crashes, bottlenecks and other traffic safety problems.

\subsubsection{Data Confidentiality}

The confidentiality assures that the messages are only read by the authorised parties. It is required in value-added services when the data sent are confidential, for example to provide secure toll payments and Internet services (DHAMGAYE; CHAVHAN, 2013). It is also required in $\mathrm{V} 2 \mathrm{~V}$ group communications, in which vehicles join the group through a shared attribute (for example, communications between police vehicles). Confidentiality is not necessary in safety applications because to the public nature of the beacons.

\subsubsection{Availability}

Availability is the provision of resources for sending any message at any time. In some scenarios such as main roads where the vehicles travel at high speed (e.g. $120 \mathrm{Km} / \mathrm{h}$ ) and if the vehicles are moving in the opposite direction, the contact time between them is very short to interchange messages (ULLAH et al., 2016; ULLAH et al., 2015). In these conditions the VANET need to guarantee the minimum delays for that the applications function correctly. On the contrary, in the case of safety applications would lead to terrible accident or a much bigger disaster.

\subsubsection{Non-repudiation}

This requirement is related to the liability attribution of an event, thus it is important for safety applications. Drivers that cause accidents should be reliably identified; a sender should not be able to deny the transmission of a message (this may be crucial in an investigation 
to determine the correct sequence and content of messages exchanged before of an accident) (RAYA; HUBAUX, 2005). Users of vehicles are liable for their deliberate or accidental actions that disrupt the operation of other nodes, or the transport system. The vehicular network should provide information that identifies or assists the attribution of liability or accountability.

\subsubsection{Privacy, unlinkability and untraceability}

In the vehicular context, privacy is achieved by means of the properties of untraceability and unlinkability. The first property states that the vehicle's actions should not be traced (e.g. different actions of the same vehicle should not be related). The second property means that should be impossible for an unauthorised entity to link the vehicle's identity with that of its driver (FUENTES; GONZÁLEZ-TABLAS; RIBAGORDA, 2010). Vehicular communication systems should not disclose or allow inferences on the personal and private information of their users. User-related privacy information includes the driver's name, licence plate, position and travelling routes. The transit authorities should be able to reveal private information in case of a car accident (LI; CHIGAN, 2014), this condition is known as conditional privacy.

\subsubsection{Trust management}

Trust management is another important component of security service in VANETs, which provides a unified approach for establishing a relationship among nodes and recording the behaviour of the vehicles (CHAURASIA; VERMA, 2013; WEI; CHEN, 2012). The traditional security mechanisms do not look after that authorised vehicles send bogus or fake messages that in the worst case put at risk the people's lives. For example, an authorised vehicle can misbehave by sending a message with false information on a supposed congested route. As a result, the vehicles will avoid travelling by that route and thus the road will be made free for benefit of the selfish vehicle (MINHAS et al., 2011). VANETs can only improve traffic safety, if the messages sent by the vehicles are trustworthy (WU; DOMINGO-FERRER; GONZÁLEZ-NICOLÁS, 2010). The trust mechanism identifies the malicious entities based on converting and extracting the detected results from security mechanisms in different systems and collecting feedback assessments continually (LEE; BAE, 2014).

\subsection{Classification of Attacks}

VANETs applications are susceptible to several kinds of threats and attacks (MEJRI; BEN-OTHMAN; HAMDI, 2014). The passive attacks can harm the confidentiality and privacy of the network, while the active attacks can damage the network resources and functioning, by inserting, deleting or modifying the exchanged messages. A legitimate vehicle within a network can be vulnerable to both external and internal attacks. The effect of external attacks remains small in comparison with the effect of internal ones (i.e., authenticated attacker). In the following, 
we analyse in detail the attacks on security requirements and their main countermeasures, as listed in Table 2.

Table 2 - Overview of attacks in VANETs

\begin{tabular}{|c|c|c|}
\hline Requirement & Attack & Countermeasures \\
\hline $\begin{array}{l}\text { Identification and au- } \\
\text { thentication }\end{array}$ & $\begin{array}{l}\text { Impersonation, replication, } \\
\text { timing, replay }\end{array}$ & $\begin{array}{l}\text { Digital Certificate accompa- } \\
\text { nying the message signature }\end{array}$ \\
\hline Integrity & $\begin{array}{l}\text { Masquerading, data alteration, } \\
\text { tampering, man in the middle, } \\
\text { spoofing GPS }\end{array}$ & Message signature \\
\hline Confidentiality & $\begin{array}{l}\text { Eavesdropping, data intercep- } \\
\text { tion, bruce force }\end{array}$ & $\begin{array}{l}\text { Encryption on sensitive messa- } \\
\text { ges }\end{array}$ \\
\hline Availability & $\begin{array}{l}\text { Jamming, flooding, sybil, mal- } \\
\text { ware, spamming, black hole }\end{array}$ & $\begin{array}{l}\text { Access control and signature- } \\
\text { based authentication }\end{array}$ \\
\hline Privacy & $\begin{array}{l}\text { Identity disclosure (unlinka- } \\
\text { bility), location tracking (un- } \\
\text { traceability) }\end{array}$ & $\begin{array}{l}\text { Pseudonyms, strategy of } \\
\text { pseudonyms changing }\end{array}$ \\
\hline Trust management & $\begin{array}{l}\text { Newcomer, inconsistency, } \\
\text { bad-mouthing, collusion }\end{array}$ & Messages signature \\
\hline
\end{tabular}

Source: Research data.

\subsubsection{Attacks on Authentication/Identification}

Every vehicle has a network identifier, which allows distinguishing it from the other nodes in the network (AL-KAHTANI, 2012). The authentication allows to the receiver vehicle to check the identity of the sender vehicle of a message; only the authenticated vehicles can access the VANETs resources and services. Indeed, external or internal attacks can be achieved using falsified identities (ZEADALLY et al., 2012b). The digital signature represents the most commonly-used method for ensuring the authentication of the vehicles. In the following, several examples of attacks on authentication are described.

i. Impersonation: the attacker obtains a valid identifier, and passes for another legitimate vehicle. This attack can be prevented by implementing proper authentication mechanisms, for example by using a Public Key Infrastructure (PKI), where each vehicle is associated with a valid certificate, which is signed by the Certificate Authority (CA);

ii. Cryptographic replication attack: keys and/or certificates are duplicated to create ambiguity. This can prevent the authorities from identifying a vehicle, especially in the case of a dispute. The first countermeasure consists of the use of certified and disposable keys to resist these attacks. 


\subsubsection{Attacks on Integrity/Data Trust}

These attacks consist of breaking the integrity of the exchanged messages by modifying, deleting, constructing or altering their content (RAWAT; SHARMA; SUSHIL, 2012). The main cryptographic solution to protect attacks on integrity consists of appending a signature to each exchanged message. However, this kind of protection cannot be applied when a data aggregation process is applied. In the following, several examples of integrity attacks are described.

i. Masquerading attack: this kind uses a valid identity (known as a mask) to ensure that it has the appearance of an authentic node, then it tries to produce false messages and broadcasts them to the neighbouring vehicles to reach specific objectives, for example to slow down the speed of a vehicle. To overcome this attack, it requires the implementation of an efficient malicious nodes detection technique;

ii. Data tampering attack: this attack can be realised by a legitimate node by fabricating and broadcasting false messages which can destroy the network and causes dangerous consequences, such as accidents (ZEADALLY et al., 2012b; PETIT; SHLADOVER, 2015). Its mechanism consists of hiding the true safety messages to legitimate users and trying to inject fake security alert messages in the network;

iii. Man-in-the-middle attack: the attacker can be a vehicle or RSU which gets inserted between the transmitter and the receiver. The attacker controls the communication between the two victims (AL-KAHTANI, 2012), while they believe that they are in direct communication with each other;

iv. GPS spoofing and injection attack: in VANETs, position information is of crucial importance and must be accurate and authentic (DHAMGAYE; CHAVHAN, 2013). This attack consists of providing to the neighbouring vehicles with false location information (PETIT; SHLADOVER, 2015). This attack is exposed in (AL-KAHTANI, 2012).

Other attacks on integrity include map database poisoning attacks and data playback attacks (MIKKI; MANSOUR; YIM, 2013).

\subsubsection{Attacks on Confidentiality}

These attacks affect only the network confidentiality and does not impact the network resources and availability (DHAMGAYE; CHAVHAN, 2013). To provide protection against this kind of attack, all sensitive data that have crucial importance should be encrypted to ensure the privacy of the vehicles and their communications (ZEADALLY et al., 2012b). In the following, several examples of these attacks are described.

i. Eavesdropping attacks: this kind of attack enables the attacker to extract sensitive information from the transmitted packets, such as the location information of the vehicles. 
This attack is dangerous and consists of listening the network traffic for a certain time and then tries to analyse the collected traffic data to extract the maximum amount of useful information;

ii. Brute force attacks: this attack can reveal the network identifier of the vehicle by using an extensive dictionary search approach. However, due to the dynamic nature of the VANETs, connection times are relatively short, and hence, a brute force attack is not easy to conduct, since it is time- consuming and resource intensive. Furthermore, this attack can become harder when it is used stronger encryption and key generation algorithms (ZEADALLY et al., 2012b).

\subsubsection{Attacks on Availability}

The Denial of Service (DoS) attack is currently recognised as the most dangerous threat to the availability, its main objective is to prevent legitimate vehicles from using the network services and resources (DHAMGAYE; CHAVHAN, 2013). Cryptographic solutions are generally not efficient to circumvent these attacks, but can limit their impact. In the following, several examples of intentional DoS and Distributed Denial of Service (DDoS) attacks are described.

i. Jamming attack: its goal is to disrupt the communication channel by transmitting noisy signals with high frequency, to decrease the Signal-to-Noise Ratio (SNR) (SUMRA et al., 2011a; TENGSTRAND et al., 2014). There are techniques to detect and mitigate this attack (TENGSTRAND et al., 2014; HAMIEH; BEN-OTHMAN; MOKDAD, 2009; MALLA; SAHU, 2013);

ii. Flooding attack: it consists of flooding the network with a huge volume of dummy messages that are intentionally generated by malicious nodes; whereby vehicles and base stations cannot communicate over the wireless channel (ROSELINMARY; MAHESHWARI; THAMARAISELVAN, 2013). This can lead, for example, to accidents if the basic safety messages are not received in time by the legitimate vehicles;

iii. Sybil attack: in this type of attack, a single vehicle (with multiple identities) gives the impression of to many vehicles. The attacker may use this type of attack to announce a traffic jam or an accident ahead. The receiver vehicle will receive the same message from different identities, and may lead the driver to change its route (R; S, 2014). Solutions to Sybil attacks are discussed in (AL-KAHTANI, 2012; WOLF, 2009; XIAO; YU; GAO, 2006);

iv. Malware: the attacker introduces malicious software in the vehicles which causes serious disruption to its normal operation. Malware attacks can be introduced by Internet, or clouds, and by Peer-to-Peer content distribution (AL-KAHTANI, 2012; DHAMGAYE; 
CHAVHAN, 2013). Such attacks can lead to dangerous consequences for VANETs and can be mitigated by using anti-virus and anti-malware software;

v. Spamming attack: its main goal is to consume the network bandwidth and introduce a high latency in the network, by sending spamming messages (e.g., advertisement messages) to a group of users. The control of this type of message is more difficult due to the lack of centralised infrastructure (DHAMGAYE; CHAVHAN, 2013; SUMRA et al., 2011b);

vi. Black Hole: the attacker refuses to participate in the network or drops out an established communication path. The network traffic may be redirected to a specific node which does not exist and cause lost of data (AL-KAHTANI, 2012; ZEADALLY et al., 2012b). This kind of attack is very dangerous for several VANETs applications, especially for latency-sensitive road safety applications;

vii. Timing attacks: it consists of delaying the transmission of latency-sensitive messages so that the safety requirements are not achieved (SUMRA; MANAN; HASBULLAH, 2011). This attack can be enabled by forcing legitimate vehicles to transmit their messages through a tunnel, which will delay the reception of these messages.

The gray hole attack is considered a variant of the black hole attack (NOGUEIRA et al., 2012). Other attacks DoS include sink- hole, worm hole (SEDJELMACI; SENOUCI, 2014), and tunnelling (RAWAT; SHARMA; SUSHIL, 2012).

\subsubsection{Attacks on Privacy}

There are several attacks on privacy; one typical attack consists of the tracking of the vehicles and/or users during their journeys (DHAMGAYE; CHAVHAN, 2013; KAUSHIK, 2013). Indeed, vehicles are generally equipped with Wi-Fi-or Bluetooth-enabled devices, which broadcast various information in clear text (e.g., identifiers, MAC addresses, device types, etc.). This information can be collected by third parties to triangulate the positions of users and track their movement within an urban environment. The main countermeasure consists of using randomised and/or temporary identifiers (e.g., MAC and IP addresses) to unlink them from the vehicles and drivers. Another approach consists of the usage of pseudonyms to provide anonymous communications (CHENG; SHAN; ZHUANG, 2011; PRIYA; KARUPPANAN, 2011).

\subsubsection{Attacks on Trust Management}

The trust management can effectively improve the peer collaboration in VANETs to share information and detect malicious peers. However, the mechanisms for trust management itself may become the target of attacks and may be compromised (ZHANG, 2011). In the following, some common attacks on trust management are listed. 
i. Self-Promoting: attackers manipulate their own reputation score by falsely increasing it. Self-promotion attacks can be performed by a single vehicle or organised groups of collaborating vehicles. The attack occurs when an attacker fabricates fake positive feedback about itself or modifies its own reputation score during the dissemination;

ii. Whitewashing: attackers evade the consequences of the misbehaviour by using some vulnerability of the system to repair their bad reputations. Once they restore their reputations, the attackers can continue the malicious behaviour. For example, the newcomer attack occurs when a malicious peer can easily register as a new user, which creates a new identification for the purpose of erasing its bad history in the network (RESNICK et al., 2000);

iii. Betrayal Attack: such attack occurs when a trusted peer suddenly turns into a malicious one and starts sending false information. A trust management system can be degraded dramatically because of this type of attacks (ZHANG, 2011);

iv. Inconsistency Attack: this attack is also called on-off attack and happens when a malicious vehicle repeatedly changes its behaviour from honest to dishonest by degrading the efficiency of the network. This attack is also similar to betrayal attack, but may be less harmful according to the study in (ZHANG; SENSOY; COHEN, 2008);

v. Slandering: reputation systems allow peers to provide feedback about other peers. Some peers may provide unfairly high feedback to increase others' reputations, which is often referred as ballot stuffing (DELLAROCAS, 2000). Some peers may provide unfairly low feedback to decrease others' reputations, which is often referred as bad-mouthing;

vi. Collusion: more than one peer in VANET may form a coalition with others to achieve a common goal. For instance, one such goal could be to give the maximum recommendation to other malicious vehicles and the minimum value to benevolent ones (MÁRMOL; PÉREZ, 2012);

\subsection{Public Key Infrastructure (PKI)}

The digital certificate accompanying the message signature is the main countermeasure against different types of attacks in VANETs, as shown in Table 2. The security architecture based on the Public Key Infrastructure (PKI) relies on asymmetric encryption/decryption algorithms to provide several security services, such as certificate generation, renewal and revocation, signing and issuing, checking and auditing. The long-term security certificate provided by a Certificate Authority in the PKI aims to link a public key with the identification of the owner. A vehicle in this infrastructure initially receives a long-term security certificate, which is only used to authenticate against third authorities in the infrastructure, and it also receives a set of pseudonyms 
from the PKI, which it will use to provide privacy and authentication against other vehicles, as will be explained in the Chapter 5 .

A full and efficient PKI is needed in VANET to attend security and privacy of the vehicles, where computing power, memory, and connection time are more constrained. Most acceptable PKI implementation for VANET is the Elliptic Curve Cryptography (ECC) (RAYA; HUBAUX, 2005). Furthermore, the standards IEEE and ETSI propose the use of the Elliptic Curve Digital Signature Algorithm (ECDSA) for message authentication. Elliptic curve-based systems can be implemented with much smaller public key sizes than the others digital signature algorithms (i.e., Rivest Shamir and Adleman- RSA, Diffie-Helman and Digital Signature Algorithm-DSA). This leads to significant performance advantages (LAUTER, 2004). ECDSA offers the same level of resistance against the best currently known attacks than others algorithms, for example, an elliptic curve over a 256-bit field currently gives the same level of security as a 3072-bit RSA/DH/DSA. The difference becomes even more dramatic as the desired security level increases, e.g., 512-bit ECC is currently equivalent in security to 15,360 -bit RSA/DH/DSA.

\subsection{Research Methodology}

This subsection has detailed information about the methodology followed in the project, see Figure 6. The methodology consisted in four stages: study of security and privacy in VANETs, design of privacy and security strategies for VANETs, execution of simulations and results analysis.

Figure 6 - Research Methodology

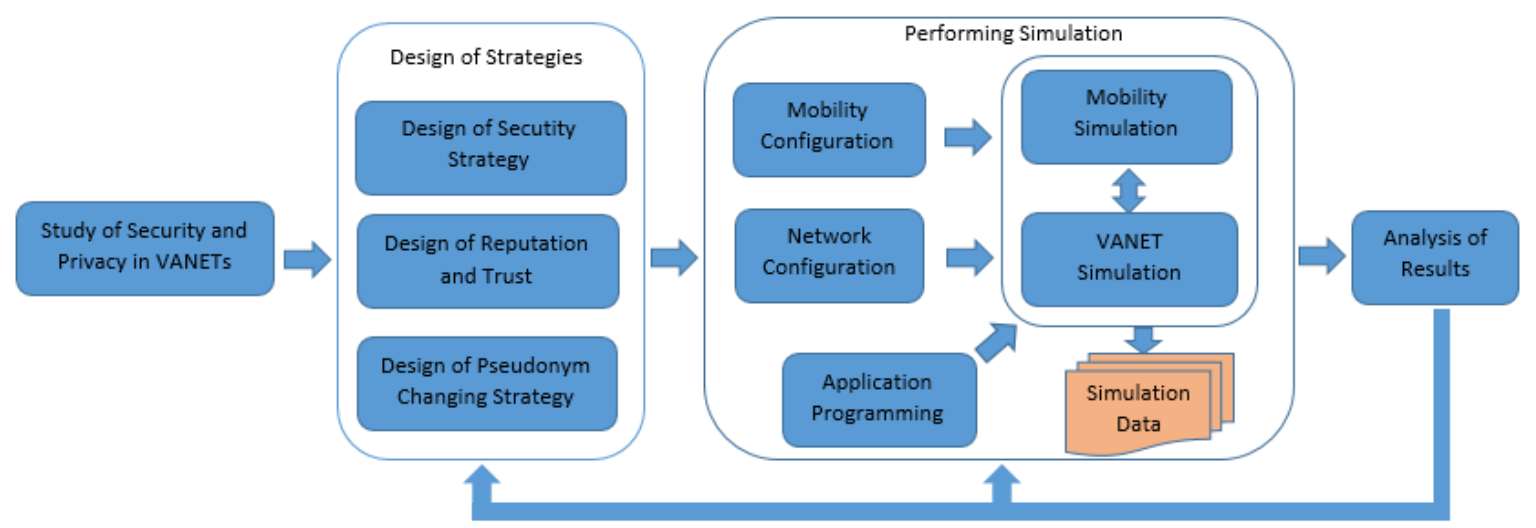

Source: Elaborated by the author.

i Study of Security and Privacy in VANETs: in this stage was realised the literature review of the aspects of security and privacy in VANETs for detecting the main problems, the architectures, models, standards, and mechanisms proposed how possible solutions. 
ii Design of security and privacy strategies for VANETs: this stage was elaborated in three sub-steps, the first sub-step consisted in the design of the security strategy based on pseudonyms, the second sub-step was focused on the design of establishing of trust in VANETs through a reputation system, and the third sub-step was the design of the pseudonym changing strategy based on reputation level.

iii Performing simulations: this stage consisted in the configuration of the vehicular mobility model and vehicular network, and programming of the application and interchange of messages which are input for a network simulator. The vehicular mobility model includes the road model, scenario parameters (i.e., maximum vehicle speed, arrival and departure rates, etc.). The vehicular network carries out a detailed packet-level simulation of the source, destinations, data traffic transmission, reception, load, route, links, and channels. The programming of the application includes the implementation of the security and privacy strategies, and the VANET application. After this, the planning of the simulations (metrics, factors, number of replies, etc.) and its execution by each one of the proposed strategies were realised. The simulations were executed in the environment shown in Section 2.9.1.

iv Analysis of results: in this stage the simulation data of each one of the experiments were graphed and evaluated according to the metrics and factors of influence defined in the planning of the experiments. Comparison of results between strategies proposed in the literature and our strategies were realised. This stage produced information for feedback the previous steps, i.e., to improve the security and privacy strategies, as well as to adjust the configuration of parameters of the simulations.

\subsubsection{Simulation Tools}

The simulation environment selected in June 2015 for this project was an integrated approach that allows the interaction between the road traffic and network simulators. Both simulators are bidirectionally coupled and simulations are performed online. In this way, the influence of vehicular networks on road traffic can be modelled and complex interactions between both domains can be examined. Figure 7 shows the set of tools used in the simulation of VANETs.

The first tool is the road traffic simulator Simulation of Urban MObility (SUMO) ${ }^{1}$, version 0.21.0. SUMO is an open source, highly portable, microscopic and continuous road traffic simulation package designed to handle large road networks (BEHRISCH et al., 2011). It is largely designed by employees of the Institute of the Transport System at the German Aerospace Center. Second tool is the network simulator Objective Modular NEtwork Testbed in $\mathrm{C}++(\mathrm{OMNET}++)^{2}$, version 4.6. OMNET++ is an extensible, modular, component-based $\mathrm{C}++$ 
Figure 7 - Simulation Tools

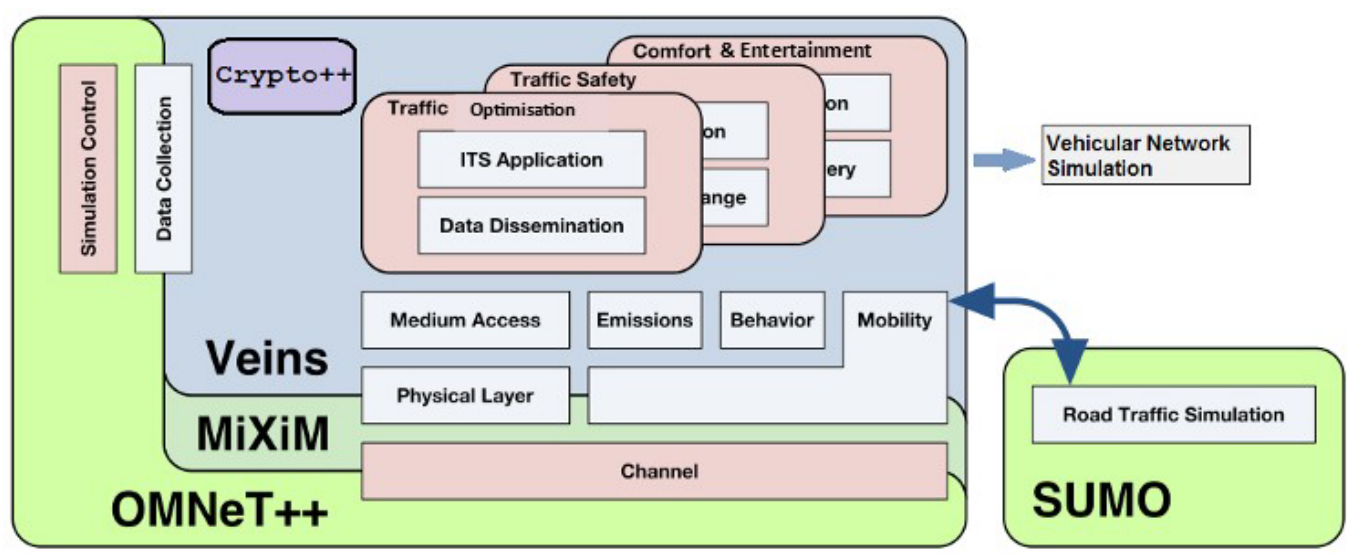

Source: Adapted from Network Simulation Tools Project Team (2016).

simulation library and framework, designed primarily for building network simulators. It offers an Integrated Development Environment (IDE) based on Eclipse, which is a graphical run-time environment, and a host of other tools. It is free for academic use and is non-profit making, and is a widely used platform in the global scientific community.

OMNET++ has several frameworks, including MIXIM ${ }^{3}$ which is used for the simulation of mobile and wireless networks. As well as the MIXIM framework, another component called Vehicular environment in network simulation (Veins) ${ }^{4}$, is required to simulate V2V and V2I communications (SOMMER; GERMAN; DRESSLER, 2011). VEINS was developed as part of an independent project for the simulation of vehicular networks, we used the version 3.0. VEINS makes possible an interaction between the road traffic and network simulators. The last tool is Crypto $++^{5}$ in its version 5.6.2, which is an open source $\mathrm{C}++$ library and offers cryptographic schemes such as Elliptic Curve Cryptography (ECC) so that it can make use of digital signatures.

Our main work was focused on developing a library of classes of security specific for VANETs called secvanet. This library imported classes from Crypto++ and implemented the pseudonym and certificate classes with the methods to sign and verify messages. The new library was imported in the vehicles to instance the pseudonyms and security certificate in the simulations. In the vehicle class was programmed the sent and reception of messages and the different security, privacy and reputation protocols as specified in the next chapters. It also was programmed the application of opportunistic forwarding of messages exposed in Chapter 4.

\footnotetext{
http://mixim.sourceforge.net/

http://veins.car2x.org/

http://www.cryptopp.com/
} 


\subsection{Final considerations}

This chapter presented some basic concepts related with the dissertation. The security aspects in VANETs differ quite significantly from the Mobile Ad hoc Networks because the high mobility and velocity of the vehicles, dynamic topology, lack of permanent infrastructure, and additional problems of privacy such as linkability and traceability. The privacy is key to secure VANETs, because lacking privacy can rise concerns about the adoption of this communication technology, delaying its widespread diffusion. One way to ensure privacy is to use a set of pseudonyms for each vehicle. A vehicle changes its pseudonyms from time to time, such that it is not possible for an intruder to know that two or more pseudonyms belong to the same vehicle.

Authentication, integrity and non-repudiation services are mandatory in VANETs, because of the liability of safety messages transmitted. In this context, the digital signature is the recommended mechanism by the security standards for protecting the VANETs communications, and it is planned to use the ECDSA by its performance advantages. Each message sent by a vehicle is authenticated using digital signature schemes, however a vehicle may misbehave due to selfish reasons and might not send right information all the time. The vehicles are driven by humans and the human behavioural tendencies are reflected in the behaviour of the vehicles. So, it is important to evaluate the trust of the received messages through the evaluation of the reputation of the sender vehicle to make decisions effective based on the received information. 
CHAPTER

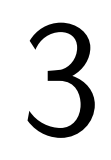

(1)

\section{A PRIVACY-PRESERVING REPUTATION SCHEME}

A mechanism for trust management is required in VANETs to record the behaviour of the vehicles, and thus, be in a position to punishing misbehaved vehicles and reward or encourage those that are well-behaved. However, it is very challenging task to handle behaviour in VANETs, featuring anonymous communications between peer vehicles or between vehicles and their infrastructure. It is complex matter to follow the historical behaviour of the vehicles, since their identities are protected by pseudonyms, which are constantly changing to prevent tracking (SUN; FANG, 2009). We adopt a centralised approach called the Privacy-Preserving Reputation Scheme (PPRS) to act as a trust management in VANETs. First, we set out the reasons for making use of a centralised architecture and describe the properties of PPRS. The novel feature of our proposal is highlighted, which is the question of the complex reputation, and geographical areas of security. After this, there is an overview of related work, in particularly with regard to centralised reputation systems for trust management in VANETs. Following this, the chapter outlines the main concepts and objects that form the building blocks of a system using PPRS, as well as describing of the organisations, agents, entities and documents involved in PPRS. The chapter goes on to explain the messages, network model, and PPRS operations in the vehicles and server through Finite State Machines. Then, the phases that must be followed for the functioning of the system and a conducting a performance analysis that takes into account factors such as processing, scalability, communications, robustness and operational costs. Basis of this chapter has been published in (JAIMES; ULLAH; MOREIRA, 2016a). Finally, the chapter concludes with some final considerations. 


\subsection{Characteristics of PPRS}

In this section, there is an outline of the reasons for employing a centralised architecture and an investigation of the properties incorporated in our reputation scheme.

\subsubsection{Centralised architecture}

A considerable effort has been made to the development of distributed trust management systems for VANETs, based on the assumption that it is not possible to rely on a centralised system for this network. A distributed system would allow an assessment to be made of the direct experience with other vehicles, although there is no guarantee that it will interact with the same vehicle in the future. Another drawback is that it is difficult to maintain the reputation history of other vehicles when each vehicle, simply changes its pseudonym and becomes a new vehicle to its neighbours. Storing information about the reputation of many vehicles will lead to scalability problems. A distributed system would also allow a list of recommendations to be made about the behaviour of other vehicles, but for the same reason, using and changing pseudonyms is a complex procedure. Owing to these factors, the distributed approaches are focused on selecting dynamic groups of vehicles and algorithms that require a considerable number of messages to be exchanged and thus could take long time to make a decision.

The first reason for adopting a centralised system is that the vehicles are regulated and governed by a centralised authority. Hence, it is natural to adopt a centralised architecture (LI et al., 2013). In addition, a centralised architecture may be preferable to a decentralised system; for example, it is often easier to manage, control, and secure a centralised system. Today, it is possible to plan a centralised reputation system, that involves new technologies such as Long Term Evolution (LTE). This could be the most efficient way of connecting the vehicles to the Internet (YANG et al., 2014) and not just depending on connections via Roadside Units (RSUs). The main advantage of a centralised system is to have a global knowledge of the behaviour of all vehicles that participate in the VANETs. The complexity of calculating the reputation score is left to the central infrastructure, which means that the vehicles can be employed in applications of greater priority.

\subsubsection{Properties of PPRS}

Our scheme incorporates the following properties which have been established by the literature for implementing an effective trust management in VANETs:

i Scalability in the VANET: the reputation system has to deal with the increasing number of nodes in the VANET and to maintain its performance. In a dense network, the number of vehicles reporting information or passing through the network can be very large. Our proposal is scalable, because the vehicle can only store its own reputation information 
and not that of other vehicles. Thus, the vehicle add its reputation information to the data messages so that it can be evaluated by their peers and do not need to query to the infrastructure for this process;

ii Security mechanisms: our scheme secures vehicular communications by providing message authentication, integrity, and non-repudiation and thus comply with the strict requirements of VANETs security standards, i.e., IEEE 1609.2 (IEEE, 2013) and ETSI TS ITS 102941 (ETSI, 2012a). These requirements include the use of digital signatures by means of the Elliptic Curve Digital Signature Algorithm (ECDSA) as mentioned in Chapter 2 (Subsection 2.8). The digital signatures in our scheme include timestamps to detect replay attacks

iii Sensitive to privacy concerns: our scheme provides privacy by means of the properties of unlinkability and untraceability. Unlinkability ensures that the verification of signed messages does not lead to the identification of their senders; and untraceability is when two or more messages sent by the same vehicle are difficult to link to each other. The reputation system has to be aware of the need for anonymous communication as a means of providing privacy to the vehicles, while building trustworthy relationships between peers. Most research studies have adopted the pseudonym approach to ensure the privacy of VANETs, see Subsection 3.5.5;

iv Robustness: the trust management itself may become the target of attacks and could thus be compromised. Measures against the main attacks are examined in Subsection 2.7.6.

$\mathrm{v}$ Apart from the properties outlined above, our scheme has the property of being a dynamic and flexible reputation system that is suited to the real conditions of the VANET. This property is achieved by defining geographical areas of security, see Subsection 3.5.3. Our scheme is also able to work with a complex reputation by including different behavioural factors (as defined in Subsection 3.5.2).

\subsection{Related Work}

In this Section, we focus on work related to reputation systems for trust management in VANETs. These are based on the architecture and, are divided into two categories. i.e., centralised reputation systems and distributed reputation systems. Firstly, we related the centralised works which were selected because included the properties of scalability, robustness and security. Table 3 compares the differences between our proposal and the centralised schemes outlined below. All these present the implementation of PKI to provide security; an important factor in the selection of ECC based PKI as object of study in our proposal is because according to IEEE 1609.2-2006 (IEEE 1609.2-2006, 2006; IEEE 1609.2-2013, 2013) standard is recommended by efficiency. ECC improves the performance of communications and storing using much smaller public key 
sizes than others digital signature algorithms with the same security strength. Secondly, we related the distributed reputation systems that were selected because considered privacy aspects in which two of them used pseudonyms.

Table 3 - Summary of literature review.

\begin{tabular}{l|l|l|l}
\hline Work & Security & Privacy & Experiments \\
\hline \hline $\begin{array}{l}\text { Single-hop reputation announcement (LI et al., } \\
\text { 2012) }\end{array}$ & $\checkmark$ & - & $\checkmark$ \\
\hline $\begin{array}{l}\text { Multi-hop reputation announcement (CAO } \text { et al., } \\
\text { 2014) }\end{array}$ & $\checkmark$ & - & $\checkmark$ \\
\hline $\begin{array}{l}\text { RGTE:A reputation-based global trust establish- } \\
\text { ment in vanets (LI } \text { et al., 2013) }\end{array}$ & $\checkmark$ & - & - \\
\hline $\begin{array}{l}\text { A Privacy-aware Reputation-based Announce- } \\
\text { ment Scheme (CHEN } \text { et al., 2013) }\end{array}$ & $\checkmark(\mathrm{BBS)}$ & $\checkmark(\mathrm{SG})$ & - \\
\hline $\begin{array}{l}\text { Private reputation retrieval in public-a privacy- } \\
\text { aware announcement scheme for vanets (CHEN } \\
\text { et al., 2015) }\end{array}$ & $\checkmark(\mathrm{BBS)}$ & $\checkmark(\mathrm{SG})$ & - \\
\hline PPRS & & & \\
\hline
\end{tabular}

Source: Research data.

BBS-Boneh, Boyen and Shacham(BONEH; BOYEN; SHACHAM, 2004), ECC-Elliptic Curve Cryptographic, SG-Signature Group, P-Pseudonym

\subsubsection{Centralised reputation systems}

The approach in (LI et al., 2012) used a single-hop reputation announcement. A reputation server collects, updates, and certifies the reputation score of the vehicles. The reliability of a message is evaluated in terms of the reputation of the vehicle that generates it. The calculation of the reputation score is based on feedback provided by other vehicles. The vehicles possess a reputation certificate which is updated by the server. In (CAO et al., 2014) proposed a multi-hop version of (LI et al., 2012), which uses the carry-and-forward method. This proposal seeks to evaluate the reliability of messages and aggregate the reputation scores. However, these two schemes lack privacy protection since the messages and feedback are linkable and not anonymous. An adversary is able to conduct an attack against the property of traceability and learn the moving of a target vehicle. They mention the use of PKI for security aspects, however they do not mention any public key algorithm in particular.

Another centralised approach called Reputation-based Global Trust Establishment scheme (RGTEs) is proposed in (LI et al., 2013). The vehicles search in its database for the reputation of the peer vehicles, and if it is not found, the vehicles query the reputation to a centralised entity through of a RSU. However, the scheme lacks confirmation through experiments, does not address privacy issues and does not mention the algorithm of public key for purposes of security. 
In (CHEN et al., 2013) a privacy-aware reputation-based announcement scheme for VANETs was proposed. This scheme relies on a centralised reputation system with an off-line trusted authority, and uses Boneh-Boyen-Shacham (BBS) group signature scheme to allow vehicles to make authenticated announcements anonymously. The security of BBS is proven in the random oracle model and relies on the Strong Diffie Hellman (SDH) and bilinear groups called the Decision Linear (DLin). The reputation is computed and updated in the server on the basis of feedback supplied by other vehicles. A secure channel is required for the retrieval of new signing keys (and hence to provide a new 'reputation status'). (CHEN et al., 2015) provide a full description of a new cryptographic primitive which enables a scheme to be designed to address a secure channel to the work in (CHEN et al., 2013). These works has three fundamental weaknesses: first, the decision whether an announcement is trustworthy or not is made by the reputation server rather than the receiver vehicle, since only vehicles deemed reputable by the server are given signing keys, and these do not reveal what the reputation scores are. Secondly, they fault of experiments. Third, the group signatures scheme in the vehicular context is limited by its overhead in terms of communication and computation.

\subsubsection{Distributed reputation systems}

In (TAJEDDINE; KAYSSI; CHEHAB, 2010), the authors used the concept of groups to make the vehicles anonymous within their groups, but still identifiable and accountable to their group managers. Each group has a reputation value that increases when the average opinion of its members is in agreement with regard to the road conditions. However, this does not lead to an individual reputation score for each vehicle, to reward honest or punish malicious vehicles. Additionally, this approach does not include an analysis of robustness. A similar work based on group formation was proposed in (CHAURASIA; VERMA, 2013). In this approach the group manager, (a pre-trusted vehicle), arranges the formation of a group of vehicles, tracks their behaviour and changes their trust values in accordance with their current behaviour. 'Pre-trusted vehicles' are police vans/cars, ambulances and other public vehicles which can be trusted by default. Factors such as robustness analysis, key size, and algorithms of the model were not specified. Its main limitation is that the formation of groups can take a long time, if pre-trusted vehicles are not present in the area. The question of with pseudonyms was not addressed.

The Joint Privacy and Reputation Assurance (JPRA) scheme mentions that each node has multiple pseudonyms for providing privacy (LI; CHIGAN, 2012). However, each pseudonym is not clearly defined. Instead, there is a procedure that ensures that the reputation information about a vehicle is always maintained by itself and among neighbours. If the network topology changes, the reputation value is partially updated with a blind signature without affecting the privacy. Additionally, a conditional discretisation algorithm for reputation was created which allow the honest vehicles to display a common reputation value. Despite this, the security information is incomplete and there are many exchanged messages due to the constant changes of topology in 


\section{VANETs.}

Another system based on pseudonyms to preserve privacy was outlined in (CHEN; WEI, 2013). In this proposal, a vehicle can make use of direct or indirect event messages and beacon messages to achieve trustworthiness values and thus be able to distinguish trustworthy event messages in the VANETs. The drawback of this approach is that the vehicle has to keep the "historical beacon information" of neighbouring vehicles, and constantly check the similarity between these beacons to confirm the trustworthiness of the vehicles. This may lead to problems of scalability, as well as increasing the storage size and processing time in the vehicles. Furthermore, the mechanism generates opinion messages that could increase the number of messages in the network. The work refers to the use of pseudonyms to provide privacy, but it is not clear how is the mapping process between the anonymous identity and the real identity of the vehicles, algorithms, key sizes, and message sizes.

\subsection{Definition of concepts and objects in PPRS}

This section examines the main concepts and objects that are the building blocks of a system that uses the PPRS. This system is described through the first three layers of a possible functional ontology in Figure 8. It is very important for VANETs to define a formal specification of conceptualisation that covers data interoperability and the attributes of reputation, security and privacy among the different agents involved in transport systems. A first approach involving an ontological representation of our reputation system was outlined in (VANNI et al., 2016). We have attempted to improve it by using as benchmarks, the concepts of the body of knowledge of the FOAF Vocabulary Specification 0.99 (Dan Brickley and Libby Miller, 2014) and PROV-O: the PROV Ontology recommended by the W3C (W3C, 2013). Certificate Authority, Reputation Authority and Traffic Regulatory Authority are demarcated as the foaf:Organization. Vehicles, Reputation Server and Roadside Unit are the main agents in PPRS, prov:Agent. Reputation, Feedback, Data Message, Geographical Area and Behavioural Factors are defined as prov:Entity. The driver and Passenger are conceptualised as foaf:Person. The Current Pseudonym, Security Certificate and Reputation Certificate are considered foaf:Document. And finally, Evaluation of Reputation and Pseudonym Changing are considered to be important activities in our scheme and described as, prov:Activity.

Figure 9 shows the main properties established in PPRS. A Vehicle is domain of the following properties: hasReputationLevel, hasReputationGlobal, hasReputationCertificate, hasSecurityReputation, hasCurrentPseudonym, hasPassenger, hasDriver and usesArea. A Data Message is the domain of the following properties: hasContent, isGeneratedBy, isForwardedBy and isAddressedTo. A Feedback isGivenBy a Observer Vehicle, evaluateTo Evaluated Vehicle, isBasedOn Content of a Data Message and isSentTo the Reputation Server. Reputation isCalculatedFrom Behavioural Factors and isUpdatedBy the Reputation Server. A Reputation Certificate 
Figure 8 - First three layers of a possible functional ontology of PPRS

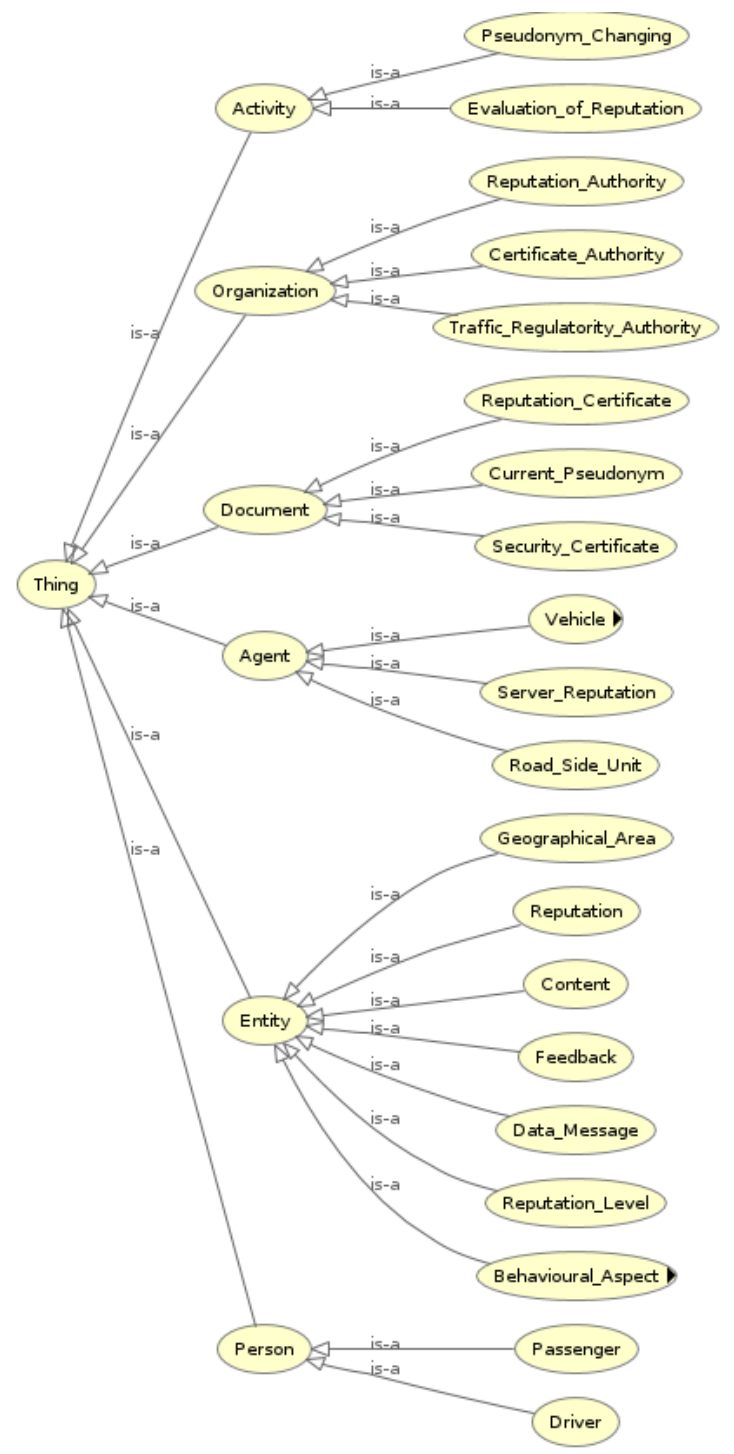

Source: Elaborated by the author.

isDownloadedFrom the Reputation Server and hasFieldReputation. The Reputation Level isDiscretisedFrom the Reputation. The Current Pseudonym isChangedBy the activity Pseudonym Changing. This activity isAssociatedWith the Vehicle. The Behavior on the Roads isReportedBy the Transit Regulatory Authority. The activity of the Reputation Evaluation dependsOn the Geographical Area, which isDeterminedBy the Reputation Server. The Reputation Authority isInChargeOf the Reputation Server. The Certificate Reputation isIssuedBy the Certificate Authority.

\subsection{Organisations and agents in PPRS}

Figure 10 shows the main organisations and agents of our scheme. In the infrastructure domain, there are the organisations of Traffic Regulatory Authority, Certificate Authority and 
Figure 9 - Main object properties of PPRS

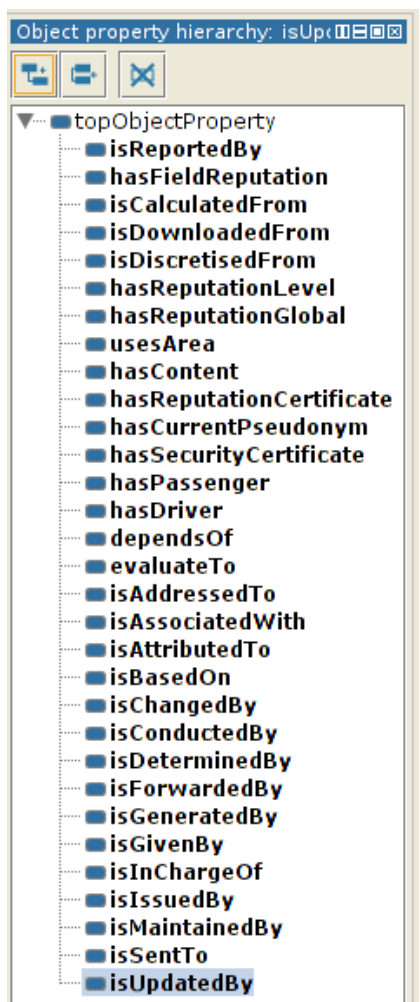

Source: Elaborated by the author.

Reputation Authority that is responsible for the Reputation Server. These entities are permanently interconnected. In the Vehicular to Everything (V2X) domain, the agent vehicles play different roles. The vehicles establish sporadic communications with the infrastructure via Roadside Units.

i Certificate Authority (CA): this is a Trusted Third Part (TTP), that operates as a traditional Certificate Authority by issuing pseudonyms to the vehicles to preserve their privacy (see definition 3.5.5). There would be a hierarchy of authorities that have a single Root CA in each administrative domain (e.g., a country) and a delegated CA in each region within that domain (e.g., a State or province) (FUENTES; GONZÁLEZ-TABLAS; RIBAGORDA, 2010). The Root CA would issue certificates to all the authorities and TTPs that could take part in VANET. In Brazil the Root CA could be the National Institute of Information Technologies.

ii Transit Regulatory Authority (TRA): this is traffic authority in each country or region that is responsible for issuing driving licence, for example in Brazil it could be the State Traffic Department (DETRAN).

iii Reputation Authority (RA): this is the organisation that administrates the Reputation Server and has the computing, communications and business infrastructure to support the reputation system. 
Figure 10 - Organisations and agents in PPRS.

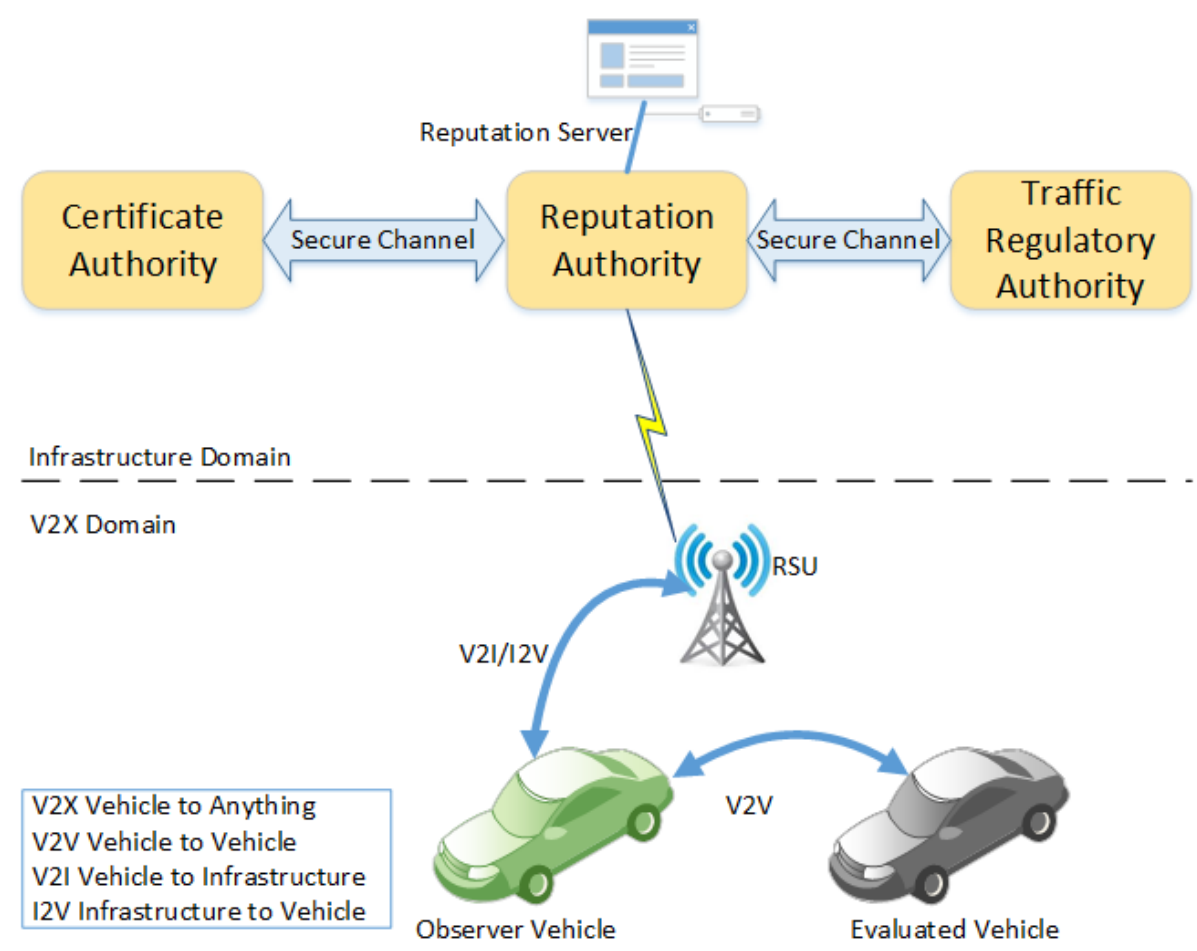

Source: Elaborated by the author.

- Reputation Server (RS): this knows the identity of the vehicles but does not have any knowledge of the pseudonyms issued to them by the CA. Thus, the RS is given feedback about the ratings, and assesses the behaviour of anonymous vehicles in VANETs. The RS maps the anonymous identities of the vehicles for the real ones, and updates the reputation scores. The functions of this server could be included in the Application Server, as in some studies (WANG et al., 2013; CHRISTIN et al., 2013). The load of the RS could be distributed to several regional servers that are combined in an interconnected national system.

iv Vehicle (V): this represents a smart vehicle as defined in Chapter 2 (Subsection 2.2.1). In our scheme, these smart vehicles can play different roles, and on the basis of this, we have divided them into the following categories.

- Evaluated Vehicle (EV): it participates in VANET and is given either positive or negative feedback from another peer or node on its behaviour in the network.

- Observer Vehicle (OV): it gives feedback to an EV based on the direct experience of interact with it, e.g., the confirmation of an event disseminated by EV. OV can either use an Internet connection to send the feedback, or an opportunistic contact with a RSU to transmit it to the server.

v Observer Node $(\mathrm{ON})$ : depending on the VANET application, other nodes that are different from those of the vehicles may play the role of observers by giving feedback to an EV. 
For example, the role of $\mathrm{ON}$ can be carried out by a central monitoring system in the infrastructure domain or a base station located on the road. ON uses an Internet connection to send the feedback to the RS.

vi Roadside Units (RSUs): these are base stations as defined in Chapter 2 (Subsection 2.2.2). The RSU acts as an intermediate node between the vehicles and the RS.

\subsection{Entities and documents in PPRS}

This section exposes the main entities and electronic documents in PPRS: reputation of a vehicle, behavioural factors of the complex reputation, geographical areas of security, Security Certificate, Pseudonyms, Reputation Certificate and feedback.

\subsubsection{Reputation of a vehicle}

The reputation of a vehicle $\left(\operatorname{Rep}_{v}\right)$ depends on its ability to maintain a score that reflects its behaviour in the VANET. For example, the reputation score could reflect the extent to which the vehicle has conveyed reliable messages in the past. The reputation score of a vehicle is the result of the aggregation of feedback by peer vehicles that evaluate its behaviour. In our centralised scheme, the Reputation Server is responsible for collecting feedback from the VANET, as well as computing and maintaining the global reputation scores of the vehicles. This means that, the reputation of a vehicle in our scheme is based on the social dimension; i.e., the direct interactions between vehicles are evaluated and sent to the server to conform the social reputation of the vehicle. The vehicles do not maintain the reputation scores of vehicles with which they have direct experiences.

\subsubsection{Behavioural factors of the complex reputation}

The reputation has always been linked to a single behavioural factor (an issue). The complex reputation makes possible to calculate reputations by different factors of behaviour. An ontological representation will help to infer the reputation based on different factors of the behaviour; Figure 11 shows what could be the complex reputation for a vehicle in our scheme. For instance, the reputation of being a vehicle that is well-behaved in the VANET, confirms its reputation of being suitable for forwarding the messages of other peers, to create its own messages, to belong to a car category, and comply with traffic regulations. The cars can be categorised in their types (Truck, Bus, Taxi, Ambulance, etc.) so that they can be further used in the context. The different factors of reputation and how they are combined to form new categories form the basis of the complex reputation of the vehicles. 
Figure 11 - Complex representation of the reputation of a vehicle

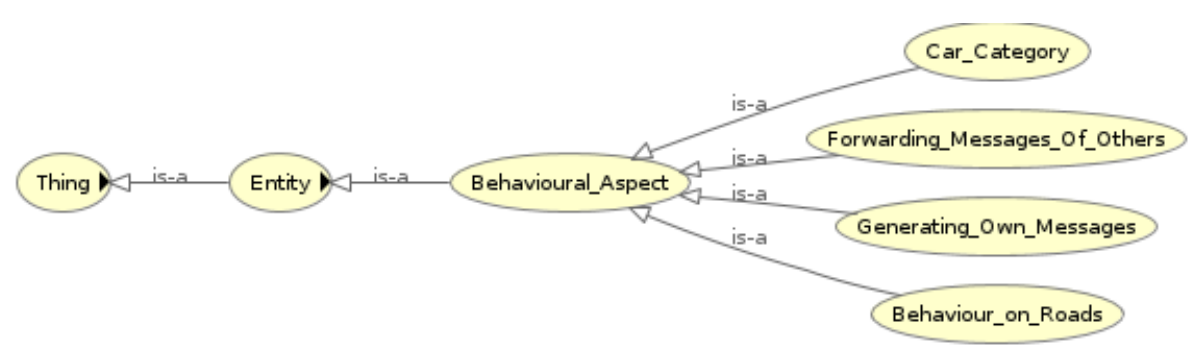

Source: Elaborated by the author.

\subsubsection{Geographical areas of security}

Our scheme provides a dynamic "reputation mechanism" through the introduction of geographical areas of security, in which the security of an area can be adapted to higher or lower levels depending on the reputation of the neighbouring vehicles that reflects the threat level that exists in the area. The RS will initially determine the areas and their risk levels. For instance, the risk level might be determined by the amount of negative feedback received from an area, this has led us to suggest classifying the areas into friendly and unfriendly. In a friendly area, the level of threat is low (few negative feedbacks) and the vehicles can disable the review of the reputation of the peer vehicles. In an unfriendly area, the level of threat is high (many negative feedbacks) and the vehicles must enable the review of the reputation of the peer vehicles. This adaptation of security is applied into the vehicles upon receipt of data messages. However, regardless the classification of the area, feedback is continuously being provided. It also continues the to operate the mechanisms of authentication, integrity, non-repudiation, and privacy.

There is at least one RSU or LTE/4G/5G base station deployed in the geographical area. This station periodically broadcasts a message giving information about the classification of the geographical area (see Area Condition Message iv). Vehicles within of the communication range of the station, receive the message, store it, and later forward it to their neighbours of one hop.

\subsubsection{Security Certificate (SC)}

The vehicles are provided with a long-term public key certificate issued by a CA, in PPRS called the Security Certificate $(S C)$. This $S C$ is used to establish secure communications between the vehicle and the RS. The $S C$ is sent to the RS when the vehicle is being registered in the Reputation System. The RS holds the public key of the vehicle for future checks on the authenticity and encryption of messages sent by the vehicle. Table 4 lists the main symbols used in our work to define aspects of security and privacy. The private key of the certificate $S C K_{v}^{-}$is used to sign messages sent from the vehicle towards the RS; and the public key of the certificate $S C K_{v}^{+}$is used to validate the integrity of the messages in the RS. 
Table 4 - Common symbols used for security and privacy

\begin{tabular}{l|l}
\hline Symbol & Description \\
\hline$S C K_{v}^{-}$ & Private key of the Security Certificate \\
$S C K_{v}^{+}$ & Public key of the Security Certificate \\
$P_{v}$ & Pseudonym of a vehicle \\
$P K_{v}^{+}$ & Pseudonym's public key \\
$P K_{v}^{-}$ & Pseudonym's private key \\
$P I_{v}$ & Pseudo-Identity of a pseudonym \\
$e d$ & Emission date \\
$C A K^{+}$ & Public key of the Certificate Authority \\
$C A K^{-}$ & Private key of the Certificate Authority \\
$A \mid B$ & Concatenation of data \\
\{\} & Digital signature \\
$\theta_{1}$ & Signature of the pseudonym \\
$\sigma_{1}$ & Signature of the data message \\
\hline
\end{tabular}

Source: Research data.

\subsubsection{Pseudonyms}

Each vehicle is equipped with a set of $n$ short-term public key certificates issued by a CA. These certificates do not contain any information identifying the vehicle, in PPRS which are called Pseudonyms $\left(P 1_{v}, P 2_{v}, \ldots, P n_{v}\right)$. The use of a single pseudonym is not enough to protect privacy, in particular the property of untraceability. Therefore, it is imperative to guarantee the privacy of the location which is achieved by frequently changing the pseudonyms used by the vehicles involved in VANET communications, as explained in Chapter 5. As in the previous work (JAIMES; ULLAH; MOREIRA, 2016b) to secure commercial advertisements in VANETs, the pseudonym is attached to the data messages to provide authentication, integrity, and nonrepudiation between the neighbour vehicles. The pseudonyms follow the specified standards in the use of the ECDSA. A pseudonym has associated a pair of keys: a public key $\left(P K_{v}^{+}\right)$and private key $\left(P K_{v}^{-}\right)$. The private key $\left(P K_{v}^{-}\right)$is employed by the vehicle to sign data messages in Vehicle to Vehicle communications (V2V). The public key $\left(P K_{v}^{+}\right)$which is included in the pseudonym, enables to verify the integrity of the data messages at the destination, as mentioned in Section 3.6. A pseudonym $P_{v}$ in our scheme is defined as,

$$
P_{v}=\left(P I_{v}, P K_{v}^{+}, e d, \theta_{1}\right)
$$

Where, the pseudo-identity $\left(P I_{v}\right)$ is a random number; emission date $(e d)$ is the date in that the pseudonym is signed by the Certificate Authority (CA); and $\theta_{1}$ is the signature on $\left\{P I_{v}\left|P K_{v}^{+}\right| e d\right\}$ with the private key $\left(C A K^{-}\right)$of the CA. The vehicles hold the public key $\left(C A K^{+}\right)$ of the CA so that they can validate the authenticity of the pseudonyms of the peer vehicles, without accessing the infrastructure. 
Table 5 - Common symbols used for the reputation system

\begin{tabular}{l|l}
\hline Symbol & Description \\
\hline$R S K^{+}$ & Public key of the Reputation Server \\
$R S K^{-}$ & Private key of the Reputation Server \\
$R C I$ & Identification of the Reputation Certificate \\
Rep & Reputation score of a vehicle \\
$K_{i} R e p$ & Reputation of the behavioural factor $i$ \\
$\theta_{2}$ & Signature of the Reputation Certificate \\
$C C$ & Check Code \\
$\sigma_{2}$ & Signature of the feedback message \\
area & It defines friendly or unfriendly area \\
$\alpha$ & Factor of weight for the last rating \\
$\delta$ & Factor of weight for a behavioural factor \\
$P T$ & Punishing Threshold \\
$C T$ & Changing Threshold \\
$l i s t$ & List of evaluated intermediate vehicles \\
$D M I$ & Data Message Identification \\
\hline
\end{tabular}

Source: Research data.

\subsubsection{Reputation Certificate (RC)}

Each vehicle is equipped with a Reputation Certificate (RC) issued and signed by the Reputation Server in the Reputation Authority. The Reputation Certificate contains the reputation score of the vehicle and is attached to the data messages so that it can report its reputation score to the neighbouring vehicles. The neighbour vehicle checks out the reputation score to decide whether to select or provide a particular service, or carry out an activity. For instance, if the sender vehicle of a message has a low score, the receiver vehicle could reject it. Otherwise, if the sender vehicle of a message has a high score, the receiver vehicle could accept it. Table 5 lists the main symbols used in our work to define aspects of reputation and feedback. A Reputation Certificate (RC) in our scheme is defined as,

$$
R C=\left(R C I_{v}, \operatorname{Rep}_{v}, e d, \theta_{2}\right)
$$

Where, $R C I$ is a unique identification that matches the real identity of the vehicle; Rep is a numerical value that represents the reputation score of the vehicle; emission date $(e d)$ is the date in that the certificate is signed by the Reputation Server (RS); and $\theta_{2}$ is the signature on $\left\{R C I_{v}\left|R e p_{v}\right| e d\right\}$ with the private key $\left(R S K^{-}\right)$of the RS.

In our scheme the reputation score of a vehicle is a value on the scale [-1,1]. The vehicles hold the public key $\left(\mathrm{RSK}^{+}\right)$of the RS to validate the integrity and authenticity of the certificates of their neighbours, without accessing the infrastructure for this. The RS sends the RC to a vehicle upon request using a secure channel, see Section 3.7. 


\subsubsection{Feedback}

The feedback represents the information that is sent to the RS to support the reputation of the vehicles. The feedback is produced by an Observer Vehicle $(\mathrm{OV})$ or Observer Node $(\mathrm{ON})$ and is a means of rating the Evaluated Vehicle $(E V)$ involved in the participation of a behavioural factor of the reputation (e.g., the generation of a data message). Our scheme attempts to monitor and update the reputation of all the vehicles involved in the message forwarding, including the use of feedback to create a meaningful reputation management system for VANETs. The PPRS defines the next elements of the feedback.

Rating: it is the numerical value that represents the evaluation from a behavioural factor (e.g., the generation of a message) given to an $E V$. In our scheme the rating can take two values, one (1) if the evaluation is positive or minus one (-1) in otherwise. For example, a vehicle could misbehave by providing false information to other vehicles about congestion on a route. A neighbouring vehicle (Observer) could find that the information is false and rate it with a rating minus one $(-1)$.

Check Code (CC): this field will help to the RS to check the authentication of the $E V s$ involved in the feedback. $C C$ is the signature on the following fields: secret_code, identification of the reputation certificate $(R C I)$ and other data (such as the Data Message IdentificationDMI). This signature uses the private key $\left(S C K_{v}^{-}\right)$of the Security Certificate of the $E V$. The secret_code is a unique number assigned to the vehicle which is known and allocated by the RS in the registration process (see, Subsection 3.10).

If the application is multihop, in each hop of the data message, the intermediate vehicle must attach its $C C$ and $R C I$ in a field called list. The RS holds the public key $\left(S C K_{v}^{+}\right)$of the vehicles with their respective RCIs. Thus, an intermediate vehicle cannot change the $R C I$ identification of an $E V$ in the list with the aim of obtaining a reputation or conspiring against another vehicle (see Subsection, 3.11.4). Thus, the vehicle or node that generates the feedback (i.e., Observer), includes the rating, list, signature, its $R C$ and $C C$.

\subsection{Messages of PPRS}

This section describes the messages used in PPRS which can make communications possible in VANETs.

i Data Message (DM): this is an application message that originates from an Evaluated Vehicle $(E V)$ and is addressed to an Observer Vehicle $(O V)$, or Observer Node. The fields of the $D M$ include a single $D M I$ identification of the message, data, the pseudonym of $E V$, the signature $\sigma_{1}$, list (Code $C C$ and $R C I$ of $E V$ ) and reputation certificate of $E V$. The 
$D M$ can be expressed as follows,

$$
D M=\left(D M I, \text { data }, P_{E V}, \sigma_{1}, \text { list }, R C_{E V}\right)
$$

Where, $\sigma_{1}$ is the signature on $\{$ DMIldata $\}$ with the private key of the pseudonym $\left(P K_{E V}^{-}\right)$. Thus, the destination of the message can check the integrity of data contained in the DM with the public key $\left(P K_{E V}^{+}\right)$extracted from the pseudonym $P_{E V}$.

The size of the message will correspond to the elliptical curve selected for applying the ECDSA. For instance, the size of the $D M$ will depend on the size of the Pseudonym, Reputation Certificate, List, and signature that are attached to the message, Figure 12. As a result of the secp $256 r 1$ curve, a digital signature has 65 bytes, and a public key has 256 bytes. Thus, a Pseudonym has 335 bytes, a Reputation Certificate has 84 bytes, and List (one hop of DM) has 75 bytes. As shown in Figure 13, the size of $D M$ is 1082 bytes with a header of 11 bytes and data of 512 bytes.

Figure 12 - General format of Data Message, $D M$

\begin{tabular}{|c|c|c|c|c|c|c|}
\hline Header & DMI & Data & Pseudonym & Signature & List & $\begin{array}{l}\text { Reputation } \\
\text { Certificate }\end{array}$ \\
\hline 11 bytes & 10 bytes & 512 bytes & $x$ bytes & y bytes & $z$ bytes & w bytes \\
\hline
\end{tabular}

Figure 13 - Specific format of Data Message, $D M$

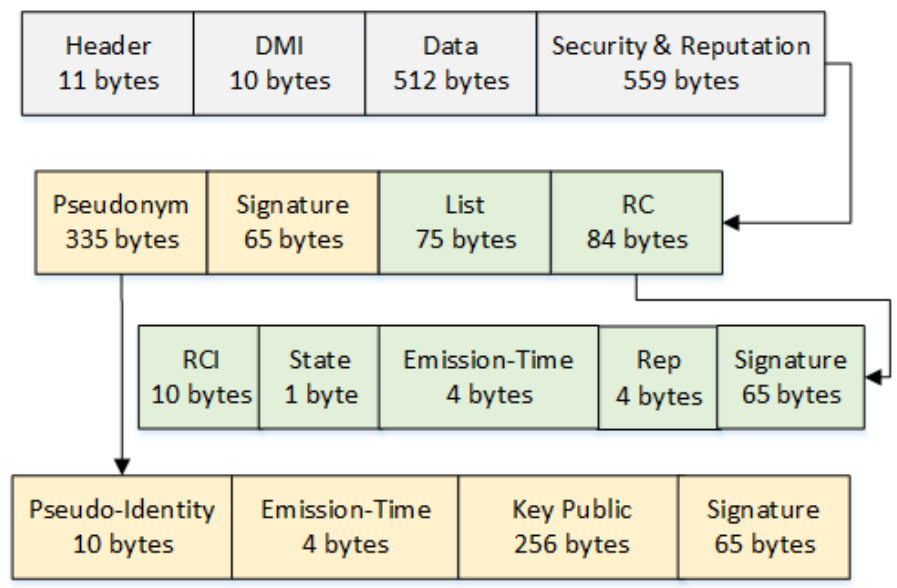

Source: Elaborated by the author.

ii Feedback Message (FM): this is a message generated by an $O V$. The message is sent to the $R S$ via a RSU or a vehicle having LTE/4G/5G connections. The fields of the $F M$ include the DMI identification of the message evaluated, list ( $R C I$ and $C C$ of $E V$ ), a rating that assesses the behaviour of the $E V$, the $\sigma_{2}$ signature, $C C$ of $O V$ and reputation certificate of $O V . F M$ can be expressed as follows,

$$
F M=\left(D M I, \text { list }, \text { rating }, \sigma_{2}, C C_{O V}, R C_{O V}\right)
$$


Where, $\sigma_{2}$ is the signature on $\{$ DMIllist|rating $\}$ with the private key of the security certificate $\left(S C K_{O V}^{-}\right)$. Thus, the RS can check the integrity of the feedback with the public key $\left(S C K_{O V}^{+}\right)$.

iii Reputation-Query Message (RQM): this is sent by a vehicle $V$ to the $R S$ via a RSU or a vehicle with $\mathrm{LTE} / 4 \mathrm{G} / 5 \mathrm{G}$ connections to request its updated $R C$. The $R Q M$ includes the pseudo-identity (PI) of the Current Pseudonym, Reputation Certificate, and the signature of the message with the private key $\left(S C K_{v}^{-}\right)$of the Security Certificate of the vehicle. The server extracts $R C I$ from the Reputation Certificate and searches the public key $\left(S C K_{v}^{+}\right)$to check the authenticity of the message.

iv Reputation-Response Message (RRM): this is generated by the $R S$ to answer to a $R Q M$. It contains the latest Reputation Certificate of $V$. The $R S$ extracts the $R C I$ identification from the Reputation Certificate and matches it with its real identity; then, it encrypts the new Reputation Certificate with the public key $\left(S C K_{v}^{+}\right)$of the Security Certificate of the vehicle. The vehicle identifies the message with its pseudo-identity (PI) and decrypts the new certificate with its private key $\left(S C K_{v}^{-}\right)$.

$\mathrm{v}$ Area Condition Message (ACM): this message is generated by the $R S$ to alert the presence of suspect vehicles in the area (i.e. an unfriendly area) or to confirm the normality of the area (a friendly area). The $R S$ periodically sends the $A C M$ through the nearby RSU to the VANET. The vehicles in the coverage range of the RSU receive the $A C M$, store it, and later forward it to their neighbouring vehicles. $A C M$ is signed with the private key of the server $R S K-$; the vehicle checks the $A C M$ with the public key of the server $R S K^{+}$.

vi SIgnaling Message (SIM): this message is periodically sent by the RSUs to alert its presence in the area to the nearby vehicles. When a vehicle receives a SIM, it examines in its cache whether it has some pending feedback to send to the server, or if its reputation certificate needs updating.

vii Hello Message (HM): this message is broadcasted by the vehicles with $D M$ messages in its cache pending by their forwarding. Its goal is to discover its neighbouring vehicles and select the next hop of the message. HM includes the Current Pseudonym of the vehicle and the peers will check its authenticity with the public key of the server $R S K^{+}$.

viii Hello Response Message (HRM): this message is sent by a vehicle as an answer to an HM. HRM includes the current Pseudonym of the vehicle and the peers will check its authenticity with the public key of the server $R S K^{+}$. 


\subsection{Network model}

Figure 14 shows our network model with the types of communication that include the following: Vehicular to Vehicular (V2V), Vehicular to Infrastructure (V2I), Infrastructure to Vehicular (I2V), and Infrastructure to Infrastructure (I2I) communications. V2V, V2I and I2V communications are carried out by means of Dedicated Short Range Communication technology (Subsection 2.5.1) and the protocol 802.11p (Subsection 2.5.3). In V2V communication, vehicles forward $D M$ messages, sending $H M$ messages, and answering $H R M$ messages. In V2I communication, vehicles send $F M$ and $R Q M$ messages to the Reputation Server through the RSU nearby. The RSU broadcasts RRM and $A C M$ messages sent by the Reputation Server to the vehicles using I2V communication. RSU also periodically broadcasts SIM messages to the vehicles using $I 2 V$ communication. The Roadside Unit identifies the messages from the vehicles addressed to the Reputation Server. After this, the Roadside Unit encapsulates these messages in IP datagrams with its IP as the source address and the IP address of the sever as the destination address. Later on, the datagrams are transmitted using I2I communications. Finally, messages from the server are decapsulated in the Roadside Unit and broadcasted to the vehicles using I2V communication.

Figure 14 - Network model.

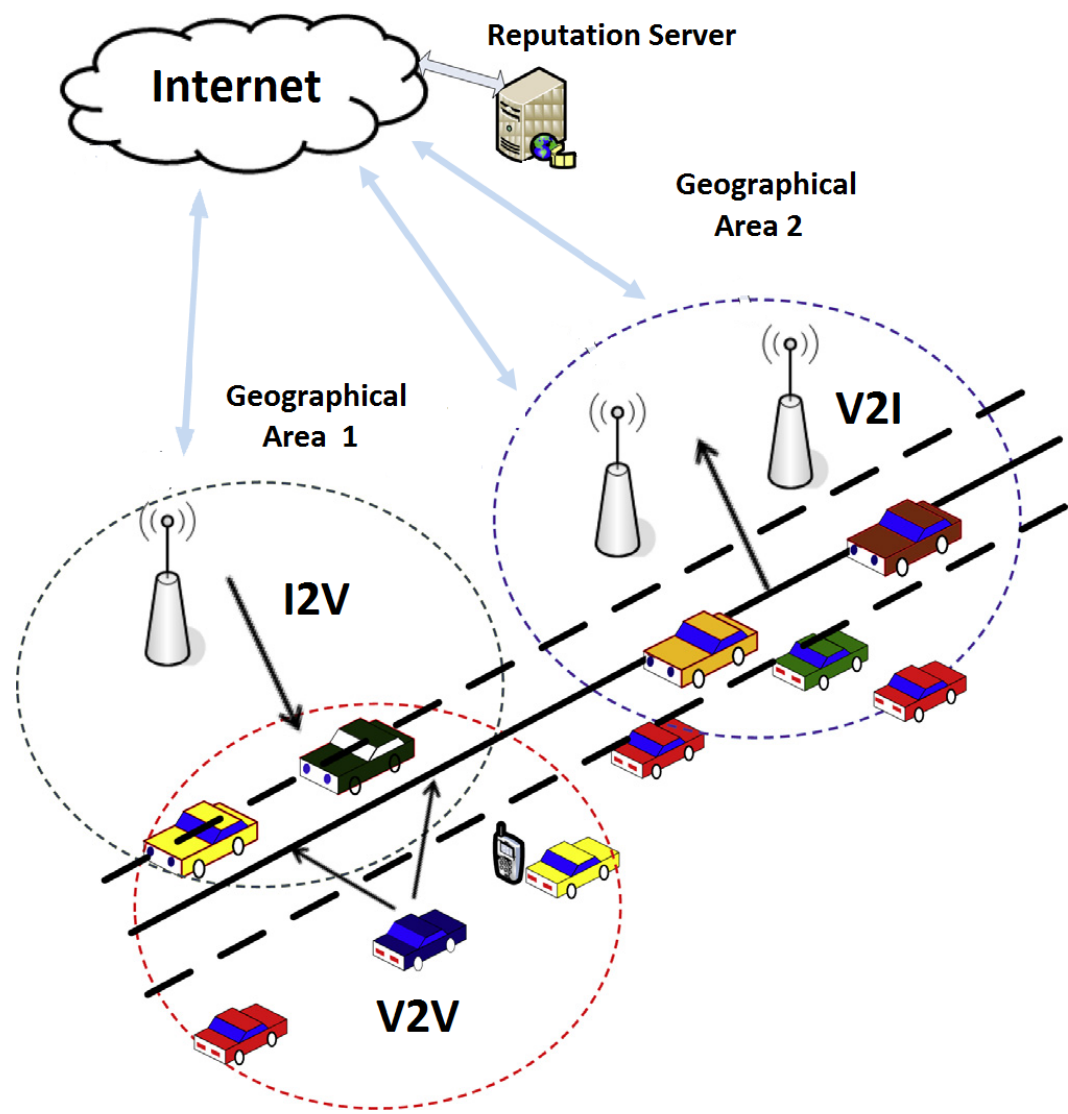

Source: Adapted from Engoulou et al. (2014). 
Figure 14 shows two geographical areas of the VANET with RSUs as gateways between the Internet and the vehicles. Secure communications between a vehicle and the Reputation Server are achieved by encrypting the content of the messages and the use of digital signatures. A vehicle signs messages to the server using the private key $S C K_{v}^{-}$of its Reputation Certificate, and the server checks the authenticity of the message coming from the vehicle with the public key $\left(S C K_{v}^{+}\right)$. The server encrypts messages going towards the vehicle using the $S C K_{v}^{+}$key. The vehicle decrypts messages coming from the server using $S C K_{v}^{-}$.

\subsection{Operation of PPRS in the vehicle}

This section explains the functions of PPRS in the vehicle that are operated through a Finite State Machine (FSM). The "primitives" used in the specification of the FSM of the vehicle are described in Table 6. PPRS operates in seven states in the vehicle, as shown in Figure 15. The vehicle starts in the Registering state where it does the entry in the RS by presenting the Security Certificate that previously acquired from the CA. Here, the vehicle acquires its initial Reputation Certificate, loads its initial pseudonym and schedules the next change of pseudonym. The vehicle from the Registering state goes to the Listening state where it waits for the reception of one of the messages (DM, RQM, SIM, HRM or ACM), or the execution of an event (confirm_evt, area_evt, forward_evt, change_evt or send_evt), or the request of the user to send Data Messages. Depending on the type of received message or event, the vehicle goes to another state.

If the message type is $A C M$, the vehicle goes to the System Updating state. The vehicle extracts the "area information" from the $A C M$; if the area is equal to zero, the vehicle enables the Friendly configuration; otherwise the vehicle enables the Unfriendly configuration. Then, the vehicle schedules the forwarding of $A C M$ to the neighbours of one hop and returns to the Listening state.

If the message type is $R R M$, the vehicle also goes to the System Updating state. The vehicle decrypts the message, extracts its Reputation Certificate from $R R M$, updates its local data, allocates the variable $r c \_t i m e$ to the time of the system (this variable controls the request of a new RC), and returns to the Listening state.

If the message type is $H R M$, the vehicle also goes to the System Updating state. The vehicle stores in cache the incoming $H R M$ message, the sender vehicle of which is the candidate to select the next hop of a $D M$. After this, it returns to the Listening state. If the message type is $S I M$, the vehicle goes to the Feedback Reporting state, where it checks into cache if there is any pending feedback to send to the server $R S$. As long as the feedback cache is not void (F represented in Figure 15), the vehicle creates and sends $F M$ messages to the $R S$. Following this, the vehicle examines the time condition for updating the Reputation Certificate. If the condition is met, the vehicle creates and sends a $R Q M$ message to request its last Reputation Certificate, and returns to the Listening state. 
Table 6 - Primitives used in the FSM of the PPRS in vehicle

\begin{tabular}{l|l}
\hline Primitive & Description \\
\hline rtd_rcv(message) & Reception of a message \\
utd_send(message) & Sending of a message \\
request_user(data) & Request of the user to send a message \\
make_pkt(parameters) & Creation and signature of a message \\
not_corrupt(message) & Verifying of the signature of a message \\
decrypt(message) & Decryption of the message \\
extract(message,field) & Extracts a field from the message \\
add-lista(message) & Adding the fields $C C$, RCI to the message \\
update(parameter) & Updating of variables \\
evaluate_rep() & Verifying of the reputation \\
select_dest() & Selection of the next hop \\
store_cache() & Storing of information in cache \\
schedule(evt) & Scheduling of a new event \\
confirm_evt() & Verifying of the truth of $d a t a$ \\
area_evt() & Enabling the forwarding of $A C M$ \\
forward_evt() & Enabling the forwarding of $D M$ \\
change_evt() & Calling for changing of pseudonym \\
send_evt() & Enabling the sent of $D M$ \\
is_observer() & Determining of the destination \\
is_true() & Determining the truth of a message \\
time() & Returning of time of the system \\
drop() & Discarding the message \\
\hline
\end{tabular}

Source: Research data.

If the message type is $D M$, the vehicle goes to the Area Checking state. If the $D M$ is addressed to the vehicle, it verifies if the configuration is Unfriendly and reviews the reputation of the sender vehicle of $D M$. The vehicle does this by extracting the $R C$ from $D M$. Then the vehicle decides whether to accept or reject the message. Otherwise, if the configuration is Unfriendly the message is accepted. After this, the vehicle determines its role; if it is an intermediate vehicle selected by a forwarding protocol (another $E V$ in the path of the message), the vehicle stores the message and schedules its forwarding. If it is an observer $(O V)$, checks the integrity of the message, and schedules the event for confirming the truth of the content of the message. Finally, the vehicle returns to the Listening state.

If the user requests a new data message to be created, the vehicle goes to the Data Sending state. The vehicle creates a new $D M$ message, creates a new $H M$ message, sends the $H M$ to its neighbours, and schedules the event send_evt(). After this, the vehicle returns to the Listening state. The vehicle also goes to the Data Sending state when it receives a send_evt(). The vehicle selects the next hop of the message between the vehicles that answered to the $H M$ and that are stored in cache, adds its data $(R C I$ and $C C)$ to the list field and sends the $D M$. Then, the vehicle returns to the Listening state. 
Figure 15 - Finite state machine of the PPRS in the vehicle.

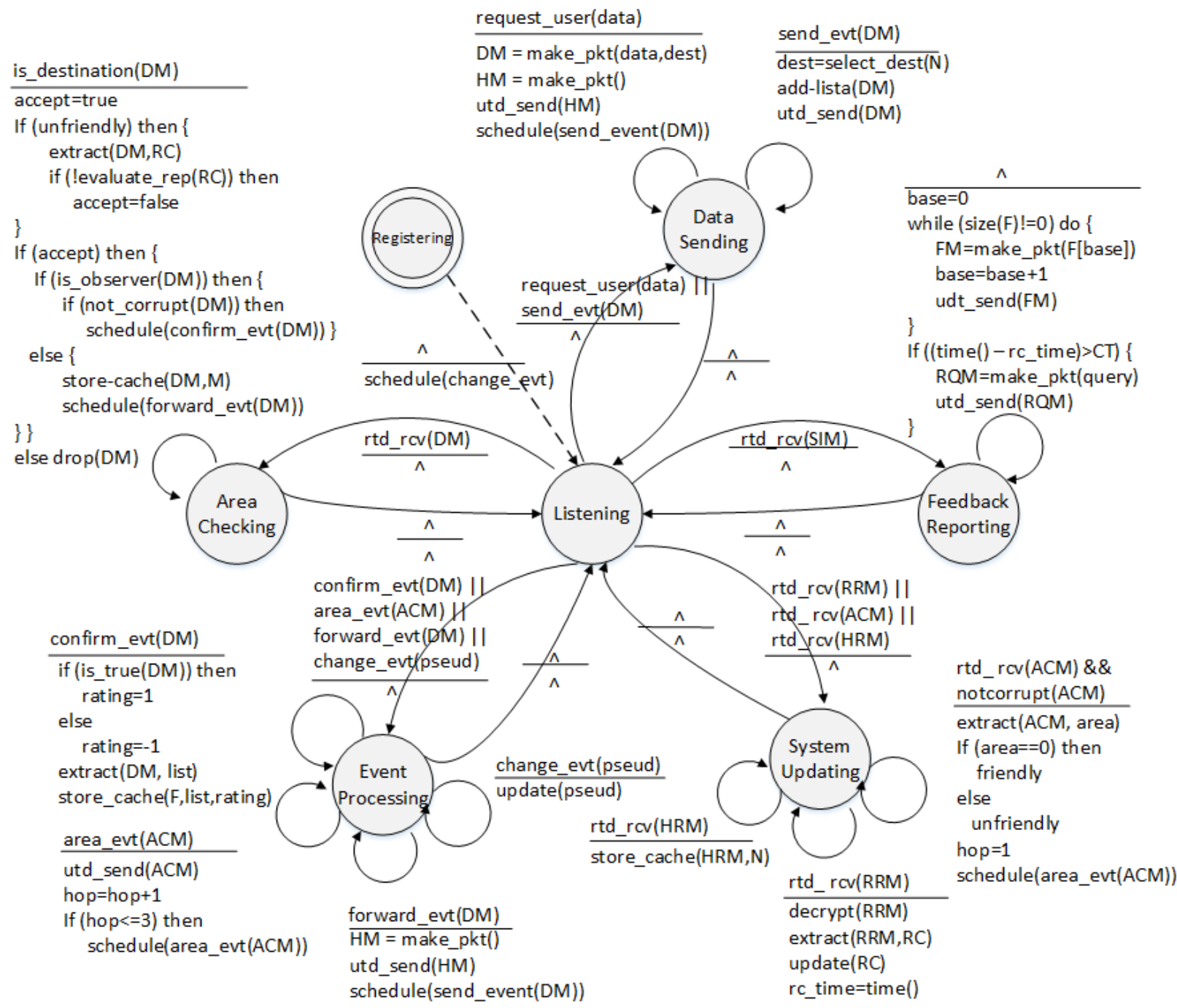

Source: Elaborated by the author.

Apart from the send_evt(), when the vehicle receives an event goes to the Event Processing state. If it is the confirm_evt(), the vehicle evaluates and confirms the truth of the content of $D M$, extracts the list of $E V s$ from $D M$, and stores in cache the corresponding feedback. If it is an area_event(), the vehicle forwards the previous ACM message, counts another hop, and evaluates the conditions required to continue scheduling the area_evt(). If it is the forward_evt(), the vehicle creates and sends a $H M$ Message, and schedules a send_evt(). Finally, if it is a change_evt(), the vehicle receives the new pseudonym and updates its current pseudonym. Then, the vehicle returns to the Listening state.

\subsection{Operation of PPRS in the reputation server}

In this section, there is an explained of the functions of PPRS in the server through a FSM. PPRS operates in five states in the $R S$, as shown in Figure 16. The server from the Starting 
state goes to the Listening state where it waits for the reception of one of the messages ( $F M$ or $R Q M)$. Depending on the type of the received message, the RS goes to different states. The new primitives used in the specification of the FSM of the server are described in Table 7.

Table 7 - Primitives used in the FSM of the PPRS in the server

\begin{tabular}{l|l}
\hline Primitive & Description \\
\hline verify () & Verification of Check Code \\
search () & Searching of Reputation Certificate \\
map () & Mapping of identity of the vehicle \\
reputation_agg( () & Executing the reputation algorithm \\
new_vehicle( $)$ & New vehicle in the system \\
register_process () & Registering of a new vehicle in the system \\
define_areas( () & Fixing the geographical areas under the server \\
behaviour() & Determining the behavioural factor evaluated \\
\hline
\end{tabular}

Source: Research data.

If the message is FM, the server goes to the Feedback Processing state. First, the message received is checked to ensure its authenticity and integrity. If the message is correct, the server extracts list and rating fields from the $F M$. Then, it starts a cycle for updating the reputation of the Evaluated Vehicles $(E V s)$ with their behavioural factors included in list. At this stage the reputation aggregation algorithm is carried out. Then, the server returns to the Listening state.

If the type of received message is $R Q M$, the server goes to the Answering state and the $\mathrm{RS}$ checks the authenticity of the message. If the message is correct, the server extracts the Check Code $(C C)$ from the $R Q M$. If the $C C$ is confirmed, the server extracts the $R C I$ identification from $R Q M$, searches the current Reputation Certificate of the vehicle, and finally, creates and sends a $R M R$ message. Then, the server returns to the Listening state.

If an area_evt() is received, the server goes to the Area Updating state. The server evaluates the condition of each geographical area in the system to determine if is unfriendly or friendly. The RS creates and disseminates an ACM message and provides information about the classification of the area through a RSU. Following this, the server returns to the Listening state. If a new vehicle enters, the registry process is carried out, the initial Reputation Certificate is sent to the vehicle, and thus the vehicle begins to take part of the system.

\subsection{PPRS Phases}

Our scheme runs in the following separate phases: 1) registration of the vehicle in the Reputation Server, 2) Sending Data Messages in the Evaluated Vehicle, 3) Evaluation of the 
Figure 16 - Finite state machine of the PPRS in the Reputation Server.

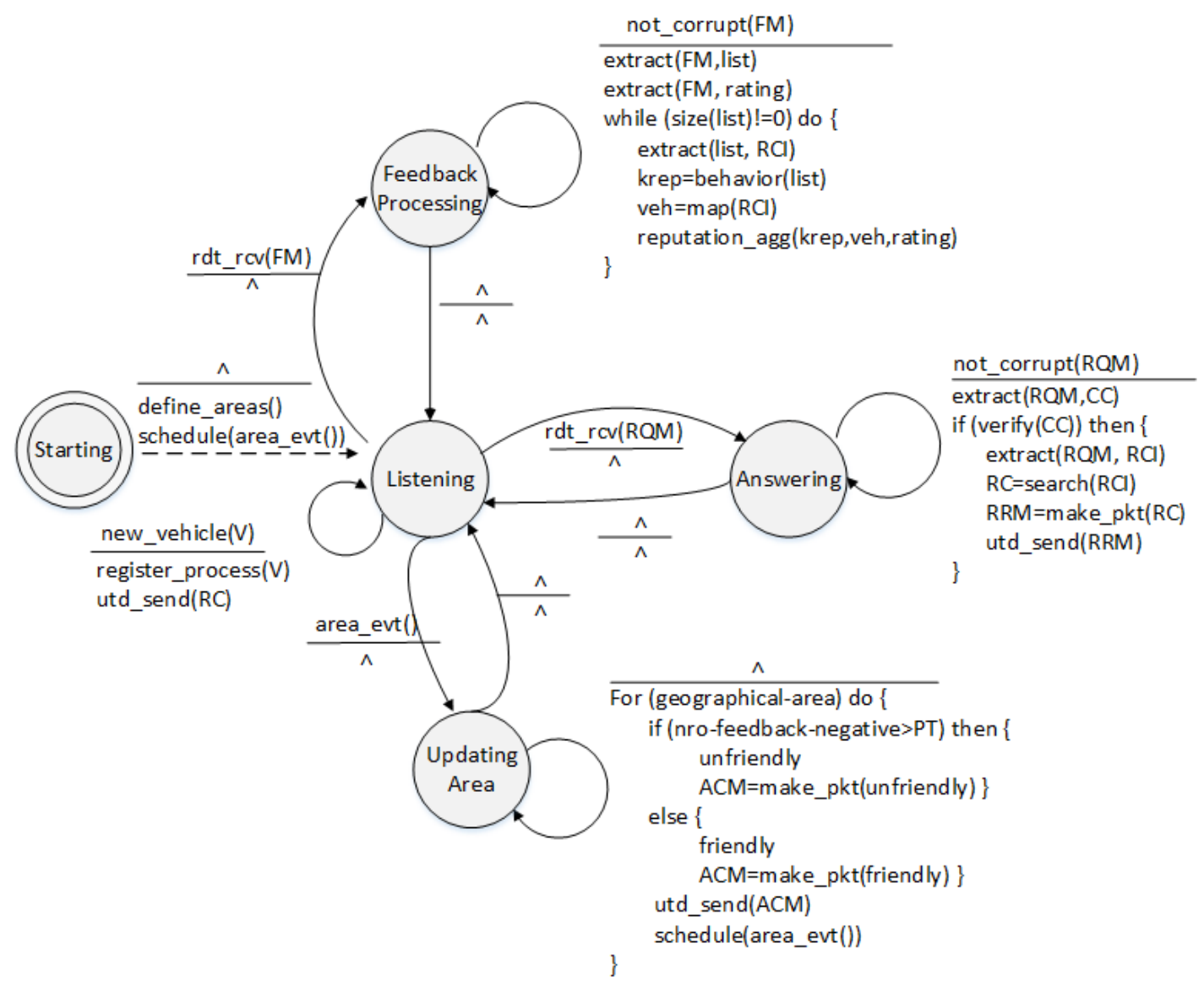

Source: Elaborated by the author.

$E V$ by the Observer Vehicle, 4) Feedback on the $O V$ sent to the $R S, 5)$ Updating reputation , 6) Obtaining the Reputation Certificate.

i Registration of the vehicle: a new vehicle does the entry in the Reputation Server by presenting the long-term Security Certificate that previously acquired from the Certificate Authority, see Figure 17. The vehicle obtains the initial Reputation Certificate with a reputation score of zero. Initially, the messages sent by new vehicles (with a zero score) will not be regarded as very reliable; however, the $O V s$ can send feedbacks on these messages. In this way, the new vehicles can gradually increase their reputation score and, hence achieve a level of trust.

All Reputation Certificates issued by the RS have a unique $R C I$ identification that matches the real identity of the vehicle. The $R S$ allocates a secret_code to each vehicle and maintains the public key of its Security Certificate for subsequent checks of messages.

ii Sending the $D M$ : in this process, the $E V$ generates and sends a new Data Message to an $O V$ destination. The content of the data depends on the nature of the application. $E V$ 
Figure 17 - Registration process of a new vehicle in PPRS.

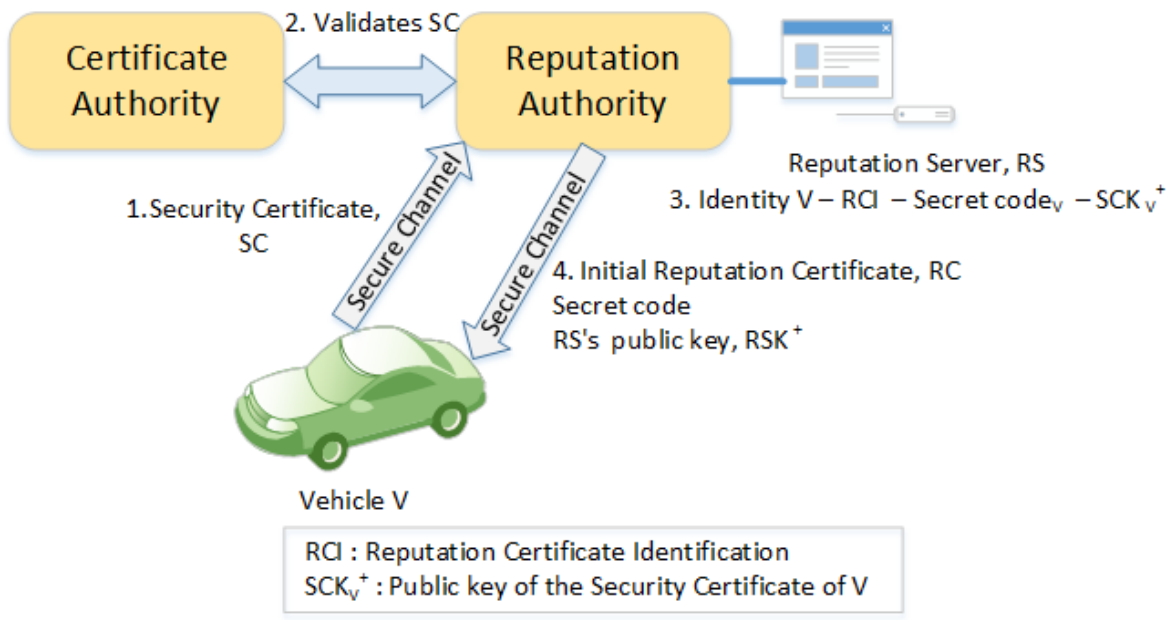

Source: Elaborated by the author.

attaches its Reputation Certificate to the message to report its reputation score to the peer vehicles. $E V$ also sends its current Pseudonym as a means of self-authentication for the neighbouring vehicles. Likewise, the $E V$ adds the Check Code to the message. If there is feedback, the code is checked by the $R S$ which knows the secret_code and the public key of the security certificate of the $E V$. The $C C$ could include the signature of other fields depending on the security specifications of the application (see Subsection 3.5.7).

iii Evaluation of the $E V$ : the Observer Vehicle $(O V)$ authenticates $E V$ by checking the signature of the Pseudonym of the $E V$ with the public key of the CA. Additionally in an unfriendly area, the $O V$ evaluates the validity and integrity of the Reputation Certificate of $E V$ by comparing its signature with the public key of the RS. After this, the $O V$ determines if the reputation score of the $E V$ is within the parameters established for accepting or rejecting $D M$. If the reputation score is within the permitted threshold of the application, the $O V$ accepts the $D M$, otherwise, it is dropped. Equally, the $O V$ checks the integrity of the data by comparing its signature with the public key of the pseudonym of $E V$. If the data has not been altered, the $O V$ embarks on a process to decide whether the the content of data was true or false.

iv Sending Feedback: the $O V$ makes an assessment of behaviour on the basis of its experience with regard to the $D M$ received from $E V$ in the previous phases. The $O V$ assesses this with a rating of 1 if the event was confirmed, or with -1 if the case is otherwise. The $O V$ generates and stores the feedback while making a connection with a RSU to send a Feedback Message $(F M)$ to the server. FM includes a list with the RCIs and CCs of the vehicles that were involved in the forwarding of the message.

$\mathrm{v}$ Updating reputation: figure 18 shows the process of mapping anonymous feedbacks in the server to provide the real identity of the vehicles with the aim of updating the reputation 
score. The server carries this out by extracting the $R C I$ of the $E V$ from the list field in the $F M$ message. Together with the RCI, it searches for the secret_code and public key of the Security Certificate registered by the vehicle. With the public key, the server is able to determine the authenticity of the Check Code issued by $E V$ and proceeds to update the reputation of $E V$.

Figure 18 - Process of identity mapping anonymous to real.

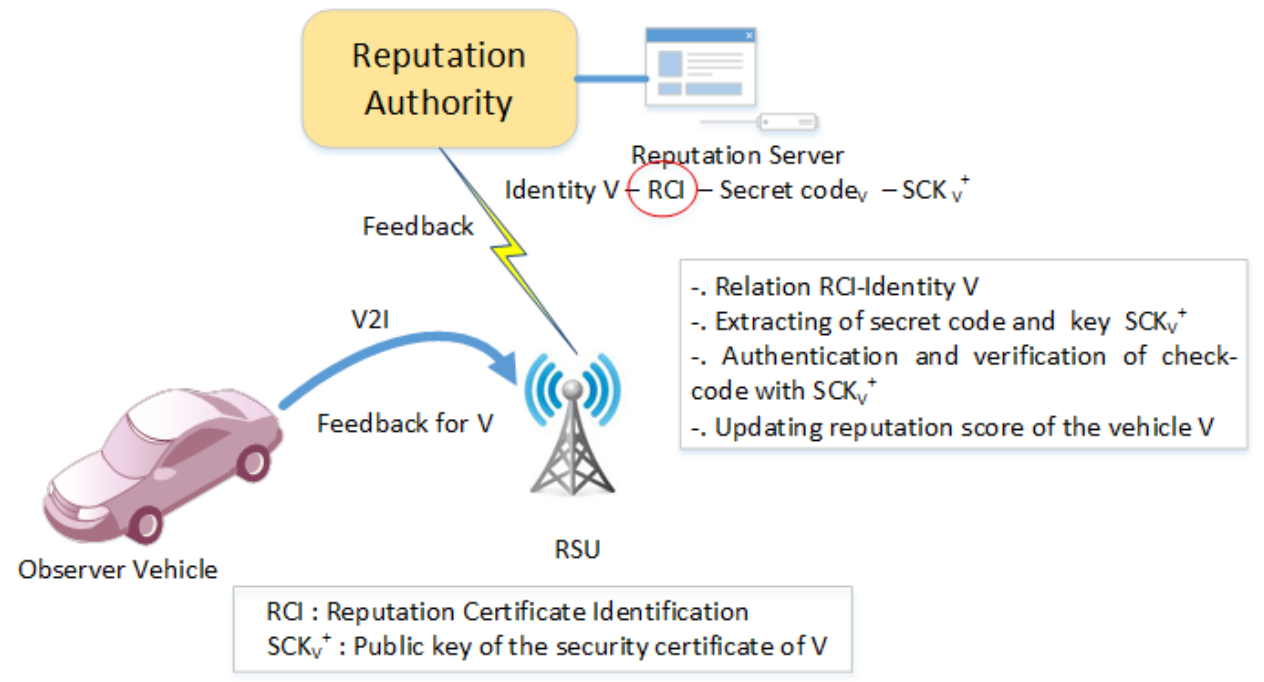

Source: Elaborated by the author.

vi Obtaining of the Reputation Certificate: the RS sends the Reputation Certificate to a vehicle in a secure manner upon request. Figure 19 shows this process, when a vehicle enters the coverage range of a base station (RSU or LTE/4G/5G), the arrival of a SIM message alerts to the vehicle of the presence of a RSU. After of this, the vehicle sends a $R Q M$ message to the RS to request its latest Reputation Certificate and finally receives the $R R M$ containing the RC.

Figure 19 - Requesting the Reputation Certificate.

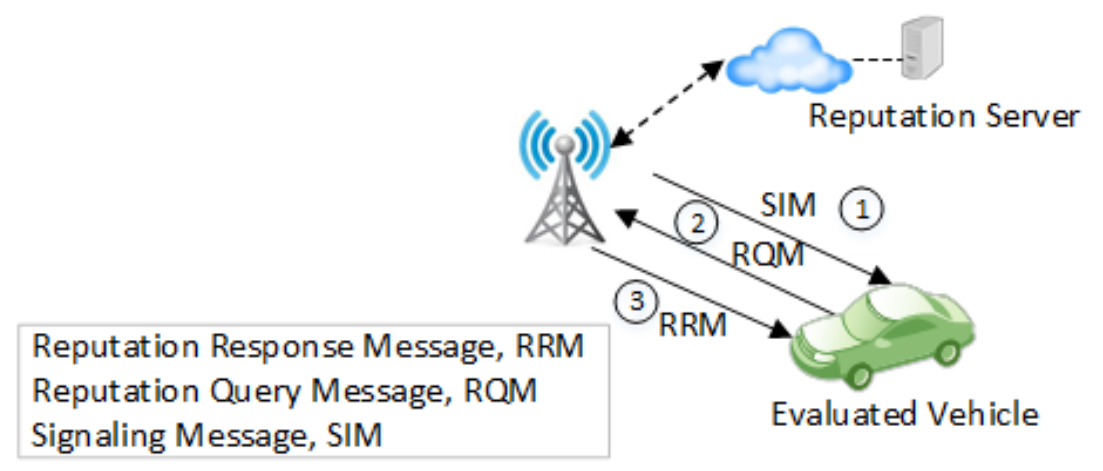

Source: Elaborated by the author. 


\subsection{Performance analysis}

This section presents the performance analysis realised on the processing time to sign and verify messages. Additionally, this section examines some key aspects of our scheme such as: scalability, communication, robustness and operational costs.

\subsubsection{Processing}

Security protocols must be implemented with a low processing time to exchange information quickly and safely. This factor is influenced by the elliptical curve selected and the kind of processor incorporated in the vehicles. Table 8 lists the size of the ECDSA key, Secure Hash Algorithm (SHA) and curve recommended for signing and checking messages in compliance with the (IEEE 1609.2-2013, 2013), (ETSI 103 097, 2015) standards, and the guidelines of the National Institute of Standards and Technology (NIST) (TURNER et al., 2009).

Table 8 - ECDSA schemes for signing and verifying of messages

\begin{tabular}{l|l|l|l}
\hline Standard & ECDSA Key (bits) & SHA & Curve \\
\hline \hline IEEE 1609.2 & 224 & 224 & secp224r1 \\
ETSI TS 103 097 & 256 & 256 & secp256r1 \\
NIST-1 & 192 & 256 & secp192r1 \\
NIST-2 & 224 & 256 & secp224r1 \\
NIST-3 & 384 & 384 & secp384r1 \\
\hline
\end{tabular}

Source: Research data.

The ECC was implemented in a computer with $2.66 \mathrm{GHz}$ Intel Core 2 Quad processor and the processing time (in ms) employed for signing and checking messages was evaluated. Our results were compared with the work in (HAMIDA; NOURA; ZNAIDI, 2015), which used two architectures Intel, of $1 \mathrm{GHz}$ and $3 \mathrm{GHz}$, see Table 9. The lowest processing time was obtained in the experiments with processor of $3 \mathrm{GHz}$.

Table 9 - Average cryptographic operation delay for the ECDSA (ms)

\begin{tabular}{l|l|l|l}
\hline \hline ECDSA/Processor & $1 \mathrm{GHz}$ & $2.66 \mathrm{GHZ}$ & $3 \mathrm{GHz}$ \\
\hline IEEE-Sign & 5.84 & 3.22 & 0.35 \\
IEEE-Verify & 34.91 & 11.43 & 1.02 \\
ETSI-Sign & 6.17 & 4.03 & 0.26 \\
ETSI-Verify & 44.03 & 16.19 & 1.22 \\
NIST-3-Sign & 14.55 & 8.12 & 0.52 \\
NIST-3-Verify & 143.63 & 34.80 & 2.98 \\
\hline
\end{tabular}

Source: Research data.

As shown in Figure 20, the processing time in a vehicle to send a Data Message corresponds to two signatures, the first signature is on the message and the second signature 
corresponds to the Check Code. The delay in the receiver vehicle, when processing a message corresponds to two checks in the friendly area, and three checks in the unfriendly area. For instance, if we selected the secp256r elliptic curve which employs keys of 256 bits and SHA-256, the processing time to send a message will be $8.06 \mathrm{~ms}$, and the processing time in the reception will be $32.38 \mathrm{~ms}$ in a friendly area, and $48.57 \mathrm{~ms}$ in an unfriendly area.

Figure 20 - Cryptographic functions for sending and receiving of messages.

a) Sent of Data Message

1. Sign ing message, $D M$

2. Sign ing Check Code, $C C$ b) Reception of Data Message, DM

1. Verifying signature of Pseudonym

2. If area is unfriendly then

Verifyng signature of Reputation Certificate

3. Verifying message DM

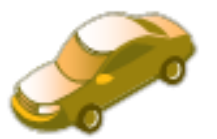

Observer vehicle, $\mathrm{OV}$

Evaluated Vehicle, EV

Source: Elaborated by the author.

\subsubsection{Scalability}

From the standpoint of storage in the vehicle, the system is scalable, the vehicle only needs to store its own Reputation Certificate. With regard to the number of Feedback messages; not all the vehicles send feedback to the Reputation Server, but only vehicles that play the role of Observers. In the transmission of Data Messages, we propose to adopt a relaying data dissemination approach, in which a single relay node is selected as the next hop, where the relay node forwards the data to the next hop and so on. The main advantage of this approach is that it reduces congestion and is scalable in dense networks. This is generally preferred for congested networks (TOMAR; CHAURASIA; TOMAR, 2010).

On the other hand, the reputation server will be designed to have the capacity to deal with and answer $F M$ and $R Q R$ messages from vehicles in a region or a small city. However, the infrastructure domain with a single server is not scalable to attend a larger VANET. It will be necessary to project a system of reputation servers interconnected to contain and distribute the reputation information of the vehicles.

\subsubsection{Communications}

The effectiveness of communications can be measured through the number of messages generated and transmitted in our scheme. The number of messages required to evaluate the reputation of a neighbour vehicle is eliminated, since that the vehicle does not need any query to 
the server or other vehicles in its communication area. Likewise, the updating of the reputation score of a vehicle does not require an exchange of messages between vehicles.

In our centralised scheme, the vehicles will be in a sporadic time online with the infrastructure and will be with frequency off-line; the effectiveness of communications will depend on the availability of Roadside Units (RSUs) in a geographical area and the number of vehicles with LTE/4G/5G connections. As mentioned in Subsection 2.4, most of the communications of our scheme will be Vehicle to Infrastructure and Infrastructure to Vehicle, and thus the optimisation of 802.11p in the area of RSU is important to obtain a minimum end-to-end communication delay. This delay will also depend on the configured bit rate, size of the messages, and the time that the server wastes in reading and answering messages coming from the vehicles. The number of messages using "Infrastructure to Infrastructure" (I2I) communications is reduced, because the mapping process of "anonymous identity to real identity" of a vehicle does not require communication between the Certificate Authority and the Reputation Server. RS directly maps the identity of the Reputation Certificate. The communication between the server and the CA is only carried out in the registration needed to authenticate the Security Certificate of the vehicle.

\subsubsection{Analysis of Robustness}

In this section, we analyse the measurements that are taken by our scheme to avoid or mitigate the different kinds of attacks. We assume that the server is protected against intruders by well-established security mechanisms as in (CHRISTIN et al., 2013). Hence, adversaries are not able to access stored data, or change the nature of the applications. Numerous attacks mentioned in Section 2.7 concerning to identity, integrity, non-repudiation and privacy, are covered by our scheme through the digital signature and mechanisms for pseudonyms. For purposes of privacy, we use pseudonyms; moreover, PPRS prevents the vehicles from taking advantage of its anonymous communications, since the transactions with the Reputation Server must be identified by a unique identification and signed with the private key of the Security Certificate of the vehicle. The Trusted Platform Module always includes a timestamp (called the emission-time in our scheme) in every signature that is generated, which makes it possible to detect replay attacks, as recommended in (PAPADIMITRATOS et al., 2008).

Here, more detail is provided about the attacks on trust management, the work in (HOFFMAN; ZAGE; NITA-ROTARU, 2009) relates the defence strategies to reputation systems. The attacks may originate from external or internal adversaries. The external adversaries are not legitimate vehicles; e.g., an attack on "feedback fraud" is not possible, unless the adversary has access to either the private key of the server or private key of the vehicle. Hence, we assume that the digital signature schemes that are used are secure, and that both the server and the vehicles manage the keys appropriately. In our scheme, malicious vehicles cannot use the Reputation Certificates of other vehicles by the inclusion of a secret_code in each signature that is only known by the vehicle and the server. Thus, a certificate can only be used by its owner vehicle. 
The internal adversaries may intend one of the following attacks against trust management:

i Self-Promoting: our scheme is able to avoid this attack, because if the Reputation Certificate is altered by a vehicle, the peer vehicle can detect the change by checking the authenticity of the certificate. The PPRS prevents the vehicles from taking advantage of its pseudo-identities for shooting Sybil attacks, since the server knows their true identities through the recognition of the Reputation Certificates and Check Codes added to the Feedback. The feedback must also be linked to one valid data message to provide accountability;

ii Whitewashing: trust models can cause these types of attacks by assigning high trust values to newcomers, so that the information provided by these vehicles is took into account by other peers when making decisions about whether to accept the information. Our scheme is able to mitigate this attack since a vehicle only needs to be registered once in the system when the first Reputation Certificate is issued. The Reputation Server only assigns an initial reputation of zero to a new vehicle.

iii Betrayal attack: the server maintains the historical feedback of the vehicles to detect this kind of attack. The server gives the minimum reputation to the vehicle that receives an amount of consecutive negative feedbacks. The system establishes validity date to the Reputation Certificate ( $r$ __time) to force the vehicles to update its certificates on a regular basis. If a vehicle does not provide updated certificates, the neighbour vehicle assumes this vehicle is untrustworthy;

iv Inconsistency attack: as in the previous attack, the server maintains the historical feedback to detect this kind of attack. The historical information should be sufficiently large to allow the server to detect unstable behaviour by a vehicle, and thus refuse to issue the next Reputation Certificate;

v Slandering: the server registers the vehicles that send feedback and establishes rules for determining if there is suspect of mutual feedback among a set of vehicles. The reputation system must solve questions about the sensitivity with regard to the estimation of negative feedback. If the sensitivity is low, the estimation will be robust against slanderous attacks on a single vehicle; however, it allows vehicles to display bad behaviour for a longer time. On the other hand, if the sensitivity is high, the bad behaviour of a single vehicle can be punished quickly, although honest vehicles are more susceptible to attacks from malicious "collectives". Our scheme which is designed to prevent false feedback includes deploying stricter feedback authentication mechanisms and validating input to make sure that the feedback is actually tied to some valid message.

vi Collusion: both the evaluated and the observer vehicles involved in feedback are checked in the server, which means that the server has the control of all the registered vehicles. 
In addition, all the data messages should be linked to a single identification, and this information must be added to the feedback. As in the case of slander attack, the server establishes rules for determining if there is a suspicion of mutual feedback among a set of vehicles that is outside the normal parameters of the system.

\subsubsection{Overall operational forecasted costs}

The costs of the reputation system mainly include the operation and administration of the Reputation Server. Initially, these costs can be covered by the manufacturers of the vehicles and the Transit Regulatory Authority. In the second place, the financial support will come from the Service Providers interested in selling products or services through the VANETs. The administration of main roads and service providers such as business owners or companies who have business alongside the main roads, might wish to advertise their services to the nearby vehicles and thus target many potential customers as in (ULLAH et al., 2016). Financial entities such as banks, credit card companies, payment systems and insurance companies will also be interested in investing in new business involving VANETs which handle sensitive information for reputation. Eventually, the vehicle owners will be able to contribute to a part of the registration costs and the annual renewal of the licence of the vehicle.

\subsection{Final considerations}

Trust management is crucial in VANETs as means of recording the behaviour of the vehicles, establishing punitive measures for misbehaving vehicles and to reward the honest vehicles. We adopt a centralised scheme for trust management based on a reputation system that is applied to VANETs applications. Our scheme is a comprehensive solution that allows vehicles maintain their privacy and security through: i) the use of pseudonyms, ii) establishing trust between peers, and iii) implementing the security mechanisms recommended by the official standards. Our scheme includes the properties for an effective trust management of VANETs: scalability, security, sensitivity to privacy, and robustness. Furthermore, PPRS adds flexibility by defining the geographical areas of security, and working with complex reputations that adopt different behavioural factors that can be represented in an ontology.

We introduced a server that was able to carry out the following functions: registering vehicles, receiving feedback, updating the reputation score of the vehicles, calculating complex reputations, punishing or rewarding their behaviour, detecting the main attacks against the trust management, defining the parameters of the system, demarcating the geographical areas of security. We introduced a vehicle that was able to carry out the following functions: generating and receiving messages, evaluating the reputation of the peers in decision-making, validating pseudonyms, checking the truth of the received data messages, generating and sending feedback, requesting its reputation certificate, and adjusting the level of security according to the threat 
risk in the geographical areas.

The vehicle holds the required security material to provide security and privacy: Security Certificate, keys, pseudonyms, public key of the Certificate Authority, public key of the Reputation Server, secret_code and Reputation Certificate. A vehicle uses the public key of the Certificate Authority to check the authenticity of the pseudonyms of the neighbouring vehicles, and uses the public key or the Reputation Server to check the authenticity and integrity of the Reputation Certificate of its peer vehicles. The communication between the infrastructure and the vehicles is hold secure through encryption of messages. The messages sent from the vehicles to the Reputation Server are digitally signed. 
CHAPTER

\section{4}

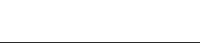

\section{AN EVALUATION OF REPUTATION WITH REGARD TO THE OPPORTUNISTIC FORWARDING OF MESSAGES}

Evaluating VANETs applications in real world environments is very expensive and requires a lot of effort. Hence, we decided to carry out simulations to validate the PrivacyPreserving Reputation Scheme (PPRS). On the basis of our previous studies in (YOKOYAMA et al., 2014; ULLAH et al., 2015; ULLAH et al., 2016), we took advantage of opportunistic encounters between vehicles to exchange data, and evaluate our scheme implemented in an opportunistic message forwarding application. A vehicle makes a decision of to accept a message based on the reputation of the sender vehicle that created or forwarded the message. This requires the Reputation Certificate of a vehicle to be attached to the messages generated or forwarded by it. The vehicles receive feedback on the behavioural factors of generating and forwarding of messages. This chapter starts by providing a description of the application. Second, we set out the motivational scenario for implementing of PPRS. Third, there is an outline of the simulation setup including mobility and network parameters, and the way the experiments were planned. We also evaluate the average reputation obtained by the vehicles, varying the number of misbehaving vehicles. Fourth, we discuss the results of the simulation experiments. Part of the results of this chapter have been published in (JAIMES; ULLAH; MOREIRA, 2016a). Finally, there is a summary of the conclusions.

\subsection{An opportunistic application for forwarding messages}

In chapter 3, we defined the general PPRS scheme to implement a centralised reputation system in VANET but did not outline a specific application. Here, PPRS is applied to an opportunistic application for forwarding messages. Everyday, there is vehicular traffic in a city which encounter other vehicles during their journeys. The frequency of these encounters is 
influenced by many factors, such as: vehicular speed, destination, traffic conditions, and the time of day. The vehicles can forward messages at a given time and the types of messages that are sent in each transaction may require a vehicle reputation score. In this case, the decision of a vehicle to accept a message is based on the reputation of the sender vehicle that created or forwarded the message. For this reason, the Reputation Certificate (Subsection 3.5.6) of a vehicle is attached to the messages that are generated or forwarded by it.

The application deals with the generation and forwarding of messages by following the store-carry and forward mechanism. Sensitive messages that inform about the traffic conditions and events occurred in the roads are generated by vehicles and sent to a central monitoring system. The message is forwarded by intermediate vehicles until it reaches the Roadside Unit (RSU). Then, the RSU sends the message to the infrastructure where is it is delivered to the final destination. The central monitoring system carries out the role of the Observer Node $(O N)$ as seen in Chapter 3 (Section iii) which provides feedback to report the truth or falsity of the content of the messages. After this, the vehicle responsible for creating the message and the vehicles involved in forwarding the message will receive feedback. As a result of the forwarding process, the $O N$ can receive several copies of the same message, but the $O N$ only generates feedback for the vehicles involved in the first copy of the message that it receives. The subsequent messages that have the same identification as the message are considered to be duplicates and are rejected.

As explained in Chapter 3 (Section iii), a vehicle may play the role of an Evaluated Vehicle $(E V)$ which is a vehicle that receives feedback. Here, we define two kinds of $E V$ : the Creator Vehicle that receives feedback as an individual agent (which generates messages) and the Forwarder Vehicle that receives feedback as a cooperative agent (which forwards messages).

i Forwarder Vehicle (FV): this is an $E V$ that is involved in forwarding the message to the RSU. When this message arrives at the central monitoring system, the $F V$ receives positive feedback, regardless of the nature of the message. Our scheme rewards the altruistic vehicles with the goal of encouraging the cooperation in the forwarding of messages. The punishing of selfish vehicles has not been the main focus in this project, however our scheme could be able to detect that vehicles are not participating in the forwarding of messages and thus reducing the reputation for this behavioural factor. Researches about the impact of selfish behaviours are being developed on systems as Peer to Peer (JIN et al., 2015) in which their solutions could be adapted to VANETs.

The operation of this application is based on the following assumptions:

i There is only one geographical area with a RSU;

ii the RSU has a canonical identification which enables it to recognise it as the destination of a message; 
iii the data messages are not private and do not need encryption;

iv the vehicles have been previously registered in the system and already have an initial Reputation Certificate.

\subsubsection{The forwarding protocol}

The forwarding protocol is executed to forward the message in the Creator Vehicle and in each hop (Forwarder Vehicle), when the vehicle is in the Data Sending state as seen in Chapter 3 (Section 3.8). It consists of three phases: neighbour discovery, selection of the next hop and the forwarding of the message. The phase of neighbouring discovery uses a HELLO-RESPONSE technique for detecting approaching vehicles (LEBRUN et al., 2005). Vehicles carrying Data Messages $(D M s)$ send out periodic Hello Message (HM). If a neighbouring vehicle hears a $H M$, it sends a Hello-Response Message (HRM) to announce its presence, as seen in Figure 21.

Figure 21 - Neighbouring discovery.

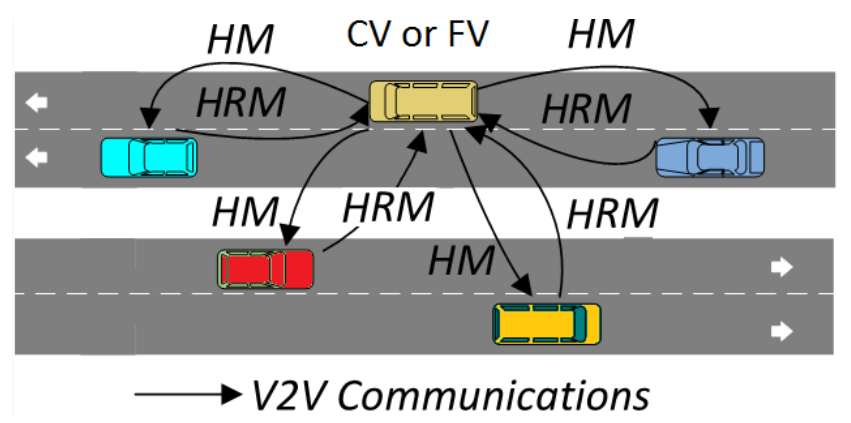

Source: Elaborated by the author.

In the selection stage, the $C V$ or $F V$ executes the algorithm which defines the next hop of the $D M$ message. The $C V$ or $F V$ listens to the $H R M$ responses for a time $t 1$; then, it determines which neighbouring vehicle should forward the message based on information about direction, position and relative velocity of the vehicles. In the third phase, the $C V$ or $F V$ forwards the $D M$ to the next hop. Finally, the next hop stores the $D M$ in the local cache and executes the forwarding protocol at $t 2 \mathrm{~s}$ to find the next hop or the destination. The message will be stored in the local cache for a maximum time of 120s. The performance evaluation of this protocol is beyond the scope of this project and can be found in (KIM; LEE, 2011).

\subsubsection{Reputation of the vehicle}

Our implementation covers a complex reputation, as seen in Chapter 3 (Section 3.5.2). Figure 22 shows the ontological structure required for inferring the reputation of the vehicle in this implementation. In this case, the reputation of being a suitable vehicle to forward a message is related to the behavioural factors for the generation of messages $\left(K_{1} \operatorname{Rep}\right)$ about road conditions and for the forwarding of messages $\left(K_{2} R e p\right)$. Thus, the vehicle can receive feedback 
for an individual behaviour with regard to the generation of messages, and/or for a cooperative behaviour with regard to the forwarding of messages.

Figure 22 - Vehicle's reputation

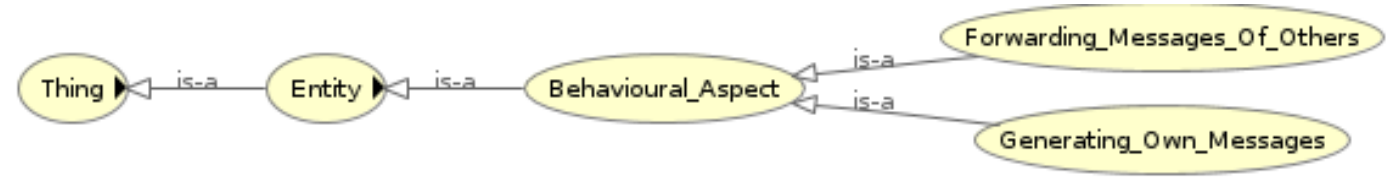

Source: Elaborated by the author.

For instance, by means of the ontological structure in Figure 22, the server calculates the reputation of a vehicle as a suitable vehicle to forward a message as follows:

$$
\operatorname{Rep}=\left(K_{1} \operatorname{Rep} * \delta K_{1}+K_{2} \operatorname{Rep} *\left(1-\delta K_{1}\right)\right)
$$

Where $\delta K_{1}$ is the weight fixed for the generation of messages and $1-\delta K_{1}$ is the weight attributed for the forwarding of messages. The weight $\delta K_{1}$ is a value on the scale [0,1]. In addition, the reputation aggregation of a behavioural factor is simply the weighted average which gives a weight $\alpha$ to the rating reported in the last feedback and gives a weight $1-\alpha$ to the historical reputation score. The weight $\alpha$ is on the scale $[0,1]$. Thus, the reputation for a behavioural factor $K i$ is,

$$
K_{i} \operatorname{Rep}=\left(K_{i} \operatorname{Rep} *(1-\alpha)+\text { last-rating } * \alpha\right)
$$

The rating receives a discrete value in our implementation because the event advertised by a vehicle is only evaluated as true or false, without possibility to intermediate assessments. Thus, it was given +1 for a true event (positive feedback) and -1 for a false event (negative feedback). As a result of the aggregation algorithm, the vehicles can obtain a reputation score on the scale $[-1,1]$, which is similar to other proposals in VANETs as the scale $[0,1]$ used in (LI; CHIGAN, 2012). Our scale meets the design goals of reputation management, which allows to reflect the behaviour of the vehicles and react quickly to abrupt changes of behaviour in the vehicles.

\subsubsection{Condition of the Geographical area}

Our implementation introduces a geographical area for controlling the classification as friendly or unfriendly. As defined in Chapter 3 (Section 3.5.2), the evaluation of the reputation of the neighbouring vehicles is "disabled" in the friendly area, and it is "enabled" in the unfriendly area. In this implementation, the classification of the geographical area as unfriendly or friendly is determined by the amount of negative feedbacks received in a given period of time. 


\subsection{Motivational scenario}

In this section, we explain our application by examining a motivational grid scenario, which is depicted in Figure 23. The vehicles enter the scenario from different origins. The RSU is deployed in the centre of the grid which is interconnected by using the Internet as a backbone. It is assumed that the RSU and vehicles are equipped with IEEE 802.11p based wireless interfaces. Additionally, each vehicle has a built-in GPS device and an OBU, with storage and processing capabilities. The RSU will broadcast SIgnalling Messages (SIM) at a regular interval, by means of beacons, and the vehicles start receiving these messages, as soon as they enter the coverage area of an RSU. In this way, the vehicles send to the RSU the Data Messages (DM) stored in its local cache to the RSU, by pending delivery to the central monitoring system. As well as this, the vehicles can update their reputation certificate from the Reputation Server through the RSU.

Figure 23 - Motivational scenario: sending and forwarding of messages in VANETs.

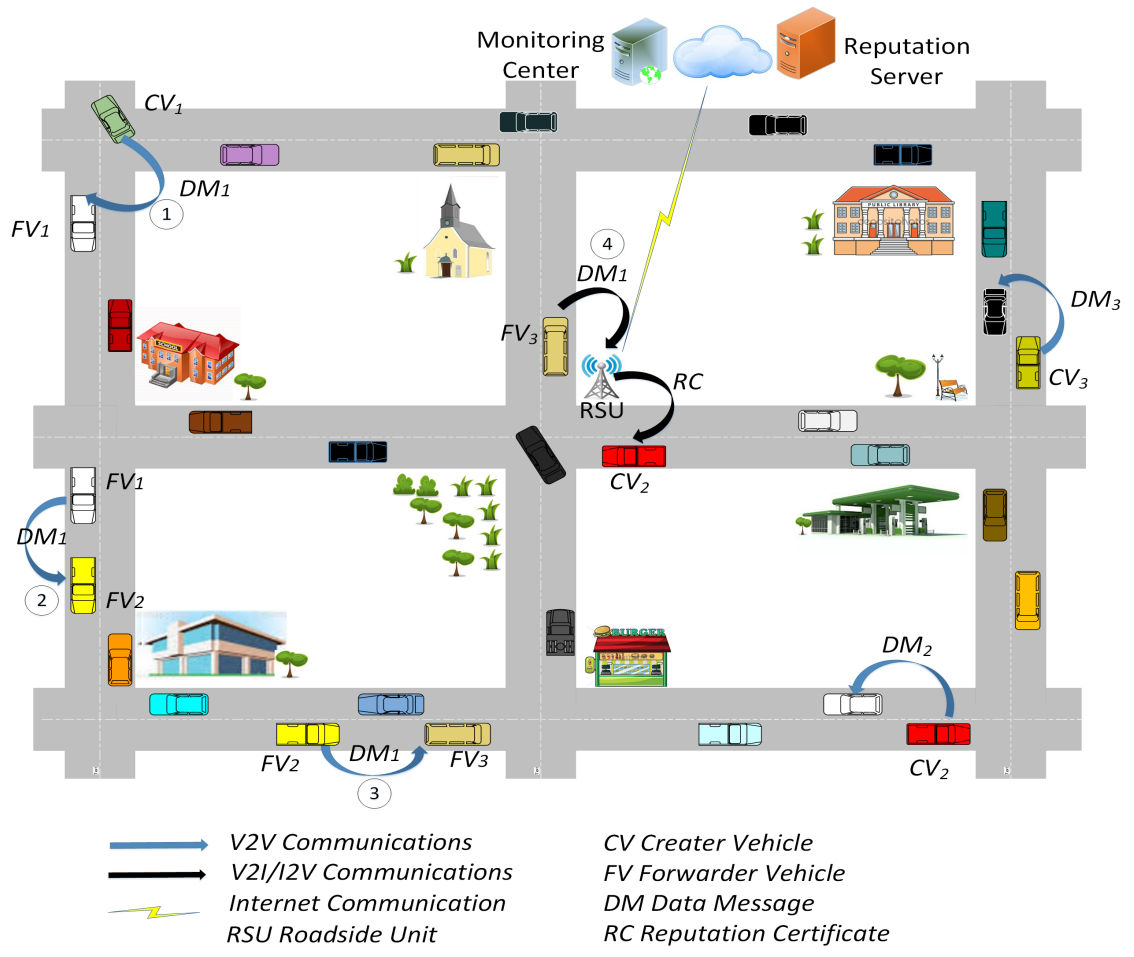

Source: Elaborated by the author.

Consider a $C V_{1}$ vehicle, which has created a $D M_{1}$ message. The $C V_{1}$ sends the message opportunistically to the $F V_{1}$ vehicle. $F V_{1}$ will store and keep the message, and later will forward it to another $F V_{2}$ vehicle. $D M_{1}$ is forwarded until it reaches the RSU which sends it to the central monitoring system. At each hop of the $D M_{1}$, the receiver vehicle extracts the reputation score from the Reputation Certificate and checks whether the reputation score of the sender vehicle is within the threshold defined by the application to accept the message. If the reputation of the sender vehicle is within the threshold of trust, the message will be accepted. Otherwise, the 
message will be rejected. The central monitoring system will give feedback to the $C V_{1}$, and the $F V s$ responsible for forwarding the message.

Now suppose that vehicle $C V_{2}$ wants to update its reputation certificate. First, it will listen to a SIgnalling Message (SM) from the RSU. Second, $C V_{2}$ will send a Reputation Query Message (RQM) to the Reputation Server. Third, it will receive a Reputation Response Message (RRM) from the server through the RSU. Finally, $C V_{2}$ will update its Reputation Certificate so that it can be joined to the Data Messages created or forwarded by it.

\subsection{Simulation Setup}

Conducting field tests or real world experiments in VANETs, especially on a larger scale, is a difficult and expensive task. It requires considerable technological effort and, laborious work, as well as involving logistical issues. Furthermore, it is also difficult to repeat the experiments with different parameters. In view of this, network simulation tools can be employed as an alternative to implement, compare, validate, and evaluate the performance of VANETrelated protocols and applications. In this section, we present the main parameters set up in the experiments and its planning. The simulation environment was exposed in Section 2.9.1.

Table 10 - SUMO configuration parameters

\begin{tabular}{l|l}
\hline Parameter & Value \\
\hline Urban area & $1 \mathrm{~km}^{2}$ \\
Number of vehicles & 100 vehicles \\
Maximum speed of $V$ & $13.9 \mathrm{~m} / \mathrm{s}$ \\
Car Model & Krauss \\
Vehicle length & $5 \mathrm{~m}$ \\
Sigma & 0.5 \\
\hline
\end{tabular}

Source: Research data.

\subsubsection{Simulation Parameters}

Parameters related to vehicles properties and a grid scenario were defined in SUMO. One of the most important parameters is vehicle speed. Additional important parameters are acceleration, deceleration, the car model, placement of RSU, scenario type and length. The parameters related to SUMO and their values are given in Table 10. Likewise, parameters relevant to OMNET++ are shown in Table 11. These parameters include transmission power, frequency band, channel, etc.

Our scheme was evaluated in an urban scenario (a grid of $1 \mathrm{~km}^{2}$ ) with five vertical and five horizontal streets, to represent a typical commercial neighbourhood, see Figure 24. A total of 100 vehicles (approximately $50 \mathrm{~km} / \mathrm{h}$ ) entered the scenario and stayed there travelling through 
Table 11 - Veins configuration parameters

\begin{tabular}{l|l}
\hline Parameter & Value \\
\hline Communication range & $250 \mathrm{~m}$ \\
Channel priorities & $\mathrm{CCH}(178)=3, \mathrm{SCH}(174)=2$ \\
Radio propagation model & Simple path loss \\
Signalling interval in RSU & $1 \mathrm{~s}$ \\
Header length & 11 Bytes \\
Beacon length & $128 \mathrm{Bytes}$ \\
Beacon interval & $3 \mathrm{~Hz}$ \\
Bit rate & $6 \mathrm{Mbps}$ \\
MAC protocol & IEEE $802.11 \mathrm{p}$ \\
Network protocol & WSMP \\
\hline
\end{tabular}

Source: Research data.

the simulation time. A RSU is deployed in the centre of the scenario and has a communication range of about 250 meters.

Figure 24 - Grid scenario for simulations.

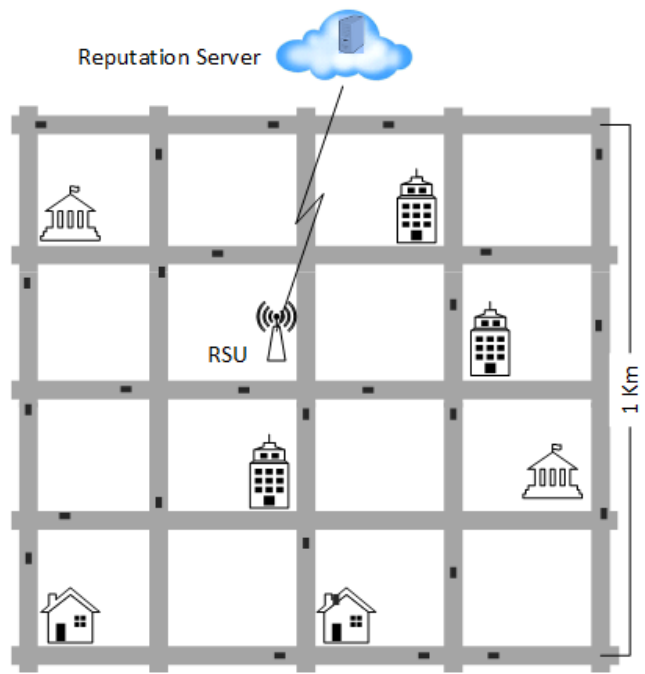

Source: Elaborated by the author.

\subsubsection{Planning the experiments}

On the basis of the works mentioned below, which included assessments of the performance of a reputation system in VANET, we selected the average reputation as main response variable in this implementation of PPRS. (MÁRMOL; PÉREZ, 2012) show variations in the percentage of malicious vehicles. The work (LI; CHIGAN, 2012) maintain a fixed number of misbehaving nodes and found that the probability of sending invalid messages varied. In the work of (DING et al., 2010), there is a randomly selected vehicle that broadcasts bogus traffic messages every 10s. (LO; TSAI, 2009) change the mobility parameters such as the maximum 
speed of the vehicles and density (LO; TSAI, 2009). In the work by (LEE; BAE, 2014) the average reputation is also calculated to determine the variation in the probability that an abnormal event has occurred.

In each experiment, we generated a representative number of messages and chose a sample of 40 Creator vehicles, a variable percentage of which were malicious vehicles as in (MÁRMOL; PÉREZ, 2012). A malicious or misbehaving vehicle is a vehicle that will always send fake messages, but will always provide correct feedback of its peers. We suppose that an event occurs every 60s around the Creater vehicles and thus, these create a Data Message, which is forwarded to the central monitoring system. Tables 12 and 13 show the experiments that were planed with the goal in mind of evaluating the impact of to have security geographical areas to check the reputation of the neighbour vehicles. We performed our simulations for $600 \mathrm{~s}$ and executed 5 runs for each experiment. The response variables and factors are defined below.

Table 12 - Planning of experiments to evaluate the impact of to change the operation mode in a geographical area

\begin{tabular}{l|l|l}
\hline Experiment & MV & Operation Mode \\
\hline 1 & $25 \%$ & Without area \\
2 & $50 \%$ & Changing area \\
3 & $25 \%$ & Constant unfriendly area \\
4 & $50 \%$ & Without area \\
5 & $25 \%$ & Changing area \\
6 & $50 \%$ & Constant unfriendly area \\
\hline
\end{tabular}

Source: Research data.

Table 13 - Planning of experiments to evaluate the impact of the weight $\delta_{k 1}$

\begin{tabular}{l|l|l}
\hline Experiment & Weight $\boldsymbol{\delta}_{k 1}$ & MV \\
\hline 1 & 0.2 & $12.5 \%$ \\
2 & 0.2 & $25 \%$ \\
3 & 0.2 & $50 \%$ \\
4 & 0.2 & $75 \%$ \\
5 & 0.5 & $12.5 \%$ \\
6 & 0.5 & $25 \%$ \\
7 & 0.5 & $50 \%$ \\
8 & 0.5 & $75 \%$ \\
\hline
\end{tabular}

Source: Research data.

\section{i Response variables}

- Average Reputation $(A R)$ : this is the arithmetic mean of the reputation score that all the vehicles have in the Reputation Server; 
- Fake Messages Percentage $(F M P)$ : this represents the number of fake messages that arrived at the destination with regard to the total number of fake messages created by the misbehaving vehicles.

\section{ii Variable Factors}

- Percentage of Malicious Vehicles (MV): this is the number of malicious vehicles with regard to the number of Creator Vehicles;

- Operational Mode: This is the configuration of the simulations with regard to the activation of the geographical areas (as seen in Chapter 3 - Section 3.5.3).

- Without area: where the vehicles are not included in any area and do not check the reputation of the sender vehicles when forwarding messages;

- Changing area: where the Reputation Server $(R S)$ and vehicles start in the friendly area, and then the $R S$ changes to an unfriendly area after detecting four negative feedbacks. $R S$ disseminates the new area conditions to the vehicles through the $R S U$. From the update of the area, the vehicles start to check the reputation score of the sender vehicles when forwarding messages;

- Constant unfriendly area: where the $R S$ and vehicles remain in the unfriendly area all the time. Therefore, from the beginning of the simulation the vehicles check the reputation score of the sender vehicles when forwarding messages;

- Weight $\delta_{k 1}$ : with a view to calculating the reputation score of a vehicle, there are two components of the reputation. Reputation by the creation and by the forwarding of messages; the first has a weight $\delta_{k 1}$ and the second has a weight $1-\delta_{k 1}$.

\section{iii Fixed Factors}

- Penetration rate: this refers to the percentage of smart vehicles registered in the reputation system; a penetration rate of $100 \%$ is assumed for the experiments;

- Initial reputation: this refers to the reputation with which the vehicle starts in the system; in our simulations the initial reputation is zero;

- Weight $\alpha$ : this is the weight given to the last rating reported in a feedback for calculating the reputation of a vehicle, and was fixed at 0.2 ; thus, $1-\alpha$ is the weight given to the historic reputation of the vehicle (i.e. 0.8);

- Elliptic curve: this refers to the type of elliptical curve used to implement the functions of Elliptic Curve Cryptographic (as seen in Section 3.11.1). We selected the secp256r curve; the functions of which include the signing and checking of pseudonyms, reputation certificates and messages. 


\subsection{Results and discussions}

In this section, there is a discussion of the results of our simulation experiments. The results were divided into four parts and henceforth, they will be explained separately in the following subsections.

\subsubsection{Evaluation of fake messages percentage, FMP}

The experiments were conducted for 40 vehicles by creating DM messages with $25 \%$ $M V$ and 50\% $M V$ (i.e., 10 and 20 Creator Vehicles sending fake messages every 60s). Figure 25 shows the results for the six experiments planned in Table12, these results of FMP on 600 fake messages in the case of $25 \% M V$ and 1200 fake messages for $50 \% M V$. In the first mode "Without area", FMP was $86 \%$ for the scenario with $25 \% M V$ and $87 \%$ for the scenario with $50 \% M V$. The main reason is that the intermediate vehicles forward all the fake messages without checking the reputation of the neighbouring vehicles. In the case of the "Changing area" and "Constant unfriendly area" modes, the results of FMP were lower than the first mode. This was because the intermediate vehicles only forward fake messages from vehicles whose reputation is greater than or equal to zero.

As we expected, the difference in the results between the second and third mode was not big. In the case of "Changing area" mode, the vehicles only start to evaluate the reputation of the neighbouring vehicles when they receive an Area Condition Message (ACM) notifying change of the area to "unfriendly" and that their Reputation Certificates have been recently updated. In contrast, in the "Constantly unfriendly area" mode, the vehicles from the beginning of the simulation check the reputation before forwarding the messages. However, it is observed in this mode that a percentage of fake messages got to the destination. This fact is due to the delay in the vehicles to travel near to a RSU and update their RCs. Consequently many vehicles present their RCs outdated with positive reputation and the fake messages are not filtered.

The results of the FMP are significant because they show the influence of a reputation system on attempts to reduce the forwarding and delivering of fake messages. As a result of the simulations, we noticed that PPRS reduces the arrival of $19 \%$ of fake messages at a destination in the scenario with $25 \% M V$; and reduces the arrival of $30 \%$ of fake messages in a scenario with $50 \% M V$. These results also demonstrate that the influence of $M V$ on the results of $F M P$ is low, the increase of $25 \% M V$ to $50 \% M V$ only got a little above messages filtered percentage in the path, in conditions where both scenarios depend on the same pattern of mobility of the vehicles to update their reputation certificates. This demonstrates system stability in filtering fake messages proportional to the number of false messages generated, i.e. 600 and 1200 respectively. Thus, by making contact with the RSU, the vehicles can update their current Reputation Certificates and the geographical area. In simulation conditions with only one RSU and no vehicle with LTE/45/5G, the percentages for FMP were high. The results could 
Figure 25 - Percentage of fake messages varying the operation mode of the system.

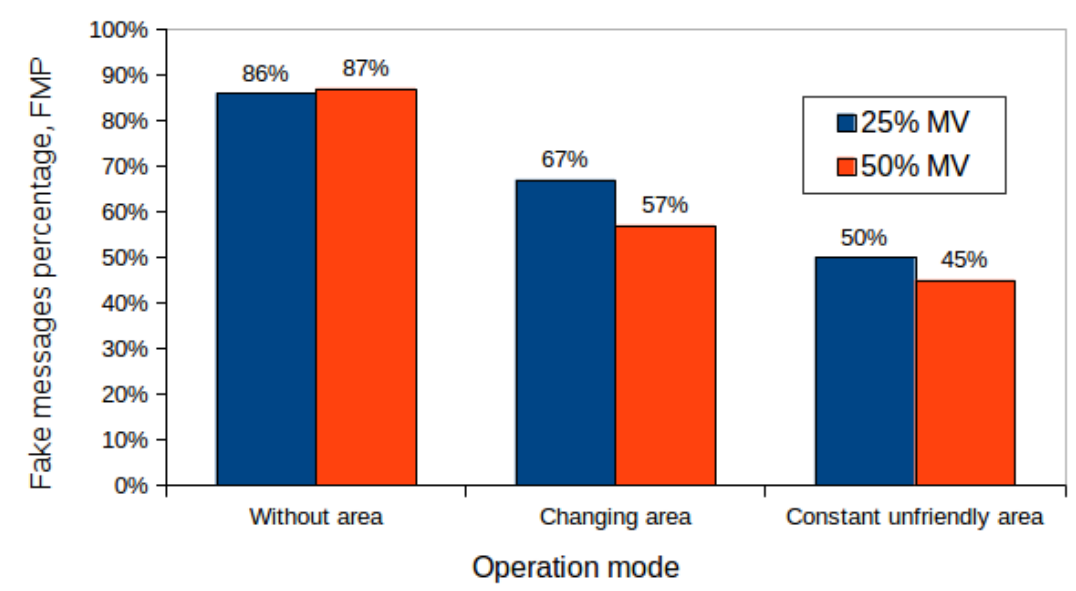

Source: Elaborated by the author.

improve if measures were taken such as guaranteeing more frequently communication between the vehicles and infrastructure. It can be concluded that it is necessary for vehicles to update their Reputation Certificates more often and thus establish a better mechanism to detect the condition of the area between friendly and unfriendly.

The results were broadened by calculating the total number of Reputation Certificate updates obtained by the vehicles minute by minute during the simulation. As shown in Figure 26, a total number of 213 updates were achieved with an average of two updates per vehicle. The number of updates per minute is low, with an average of 21 vehicles being updated per minute. The percentage of FMP is influenced by the opportunistic contact of the vehicles with the RSU, as a means of sending feedback and updating their Reputation Certificates. This is because the vehicles start to filter messages when they recognise an unfriendly zone and the vehicles attach its Reputation Certificate to the messages, which has been updated with the latest reputation score.

\subsubsection{Average Reputation (AR) in a scenario with low weight assig- ned for the generation of messages}

In this section, we analysed the first four experiments in Table 14, which the weight $\delta_{k 1}$ was fixed at 0.2 and the percentages of malicious vehicles $(M V)$ were fixed at $12.5 \%, 25 \%, 50 \%$ and $75 \%$ respectively. Figure 27 shows the four scenarios of $M V$ with the results of the average $(A R)$ minute by minute deployed as: total, for the generation of messages and for the forwarding of messages. The total $A R$ is calculated from Equation 4.1.

Figure 27a shows the results of the $A R$ for the scenario with $12.5 \%$ of the $M V$ (i.e., 5 of the Creator Vehicles sending fake messages). The $A R$ for the generation of messages is positive and less than 0.1 . The main reason is that the number of vehicles sending honest messages is 
Figure 26 - Total results of reputation updates

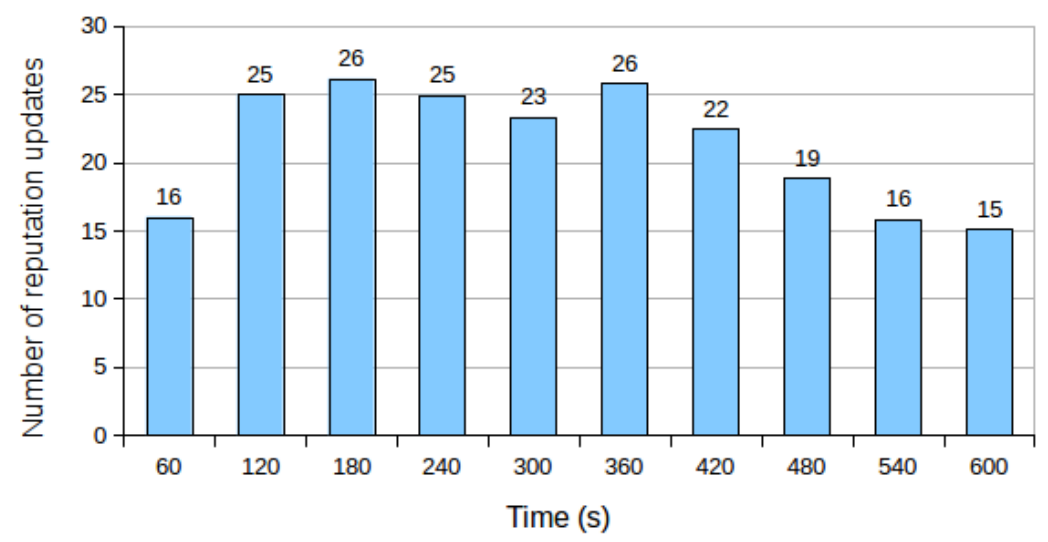

Source: Elaborated by the author.

Figure $27-$ Results of $A R$ varying $M V$ with $\alpha=0.2, \delta_{k 1}=0.2$ and $1-\delta_{k 1}=0.8$

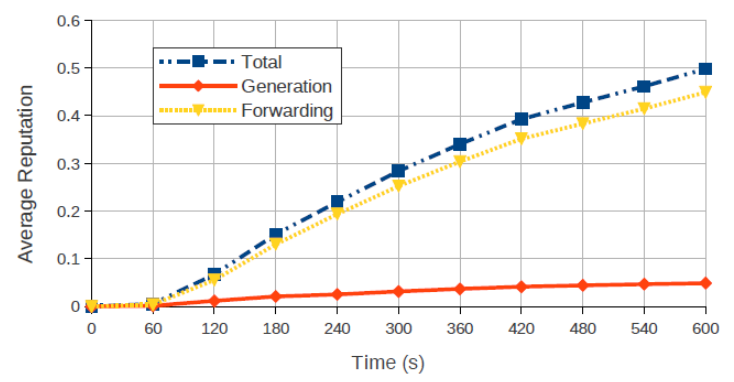

(a) $\mathrm{MV}=12.5 \%$

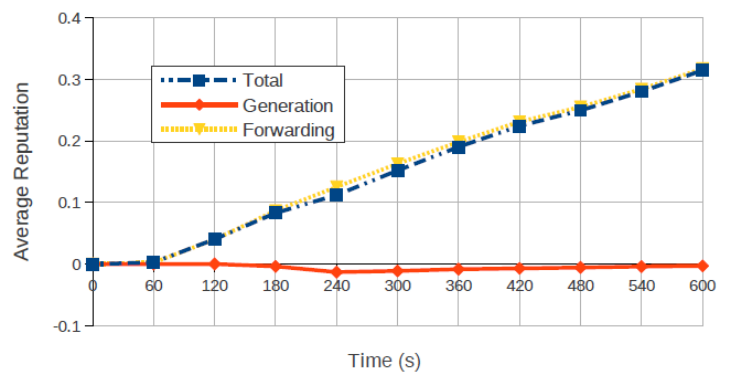

(c) $\mathrm{MV}=50 \%$

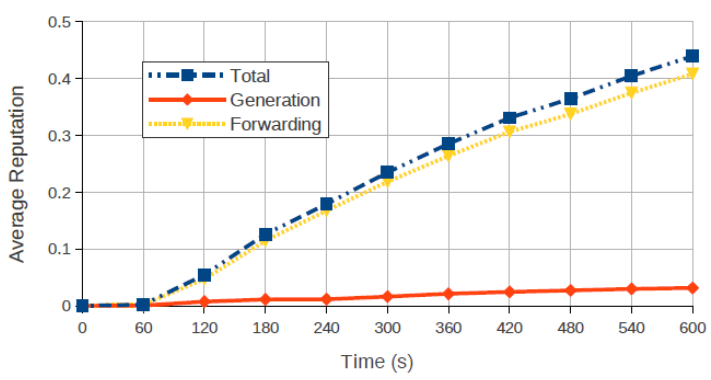

(b) $\mathrm{MV}=25 \%$

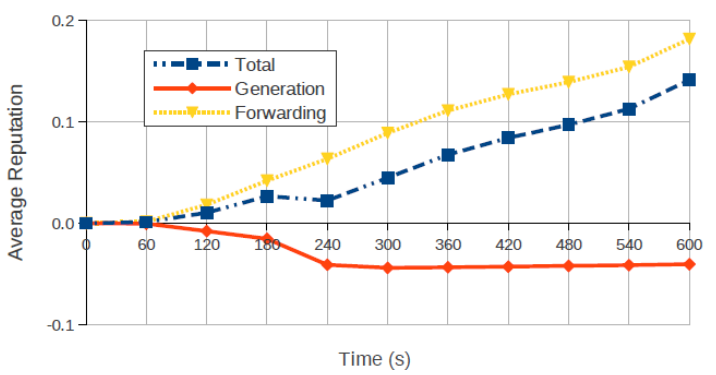

(d) $\mathrm{MV}=75 \%$

Source: Elaborated by the author.

greater than the number of vehicles sending fake messages. $A R$ for the forwarding of messages is also positive and close to the results of the total $A R$. This is because the total $A R$ is mainly influenced by the behavioural factor that has the greatest weight and in this case involve the forwarding of messages. Figure $25 \mathrm{~b}$ shows the results of the $A R$ for the scenario with $25 \%$ of the $M V$ which are similar to the results of the scenario with $12.5 \%$ of the $M V$.

Figure $27 \mathrm{c}$ depicts the results of $A R$ for $50 \%$ of the $M V$; unlike the scenarios mentioned above, the values of the $A R$ for generation of messages are almost zero, because only half of the 
vehicles send honest messages and the other half send fake messages. For the same reason, when the generation of messages have almost zero influence, the results of the $A R$ by the forwarding of messages are very near to the overall results.

Figure $27 \mathrm{~d}$ represents the results of $A R$ for $75 \%$ of the $M V$. The $A R$ for generation of messages decreases the first minutes until the fourth minute when the Reputation Server detects 4 negative feedbacks because of the arrival of fake messages at the destination. This situation of receiving fake messages continues until the end of the simulation. $A R$ for forwarding messages is positive, less than the total $A R$ and slightly increases minute by minute. The main reason is that only 10 Creator Vehicles are sending honest messages. The results for the generation of messages did not drop to -0.2 for this case, because the Malicious Vehicles were also able to receive positive feedback for the forwarding of messages.

\subsubsection{Average Reputation (AR) in a scenario with medium weight assigned for the generation of messages}

In this section, we analysed the last four experiments in Table13. Likewise in the first set of experiments, we evaluated the performance by considering the same percentages of malicious vehicles $(M V)$ at $12.5 \%, 25 \%, 50 \%$ and $75 \%$ respectively. However, we changed the weight assigned for the generation of messages $\delta_{k 1}$ to 0.5 . The results of the $A R$ obtained are shown in Figure 28a- 28d.

Figure $28-$ Results of $A R$ varying $M V$ with $\alpha=0.2, \delta_{k 1}=0.5$ and $1-\delta_{k 1}=0.5$

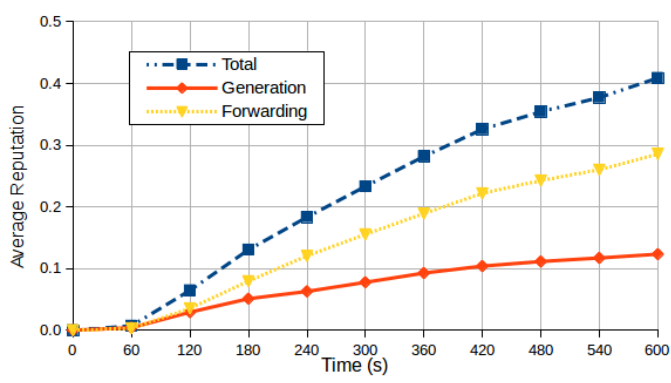

(a) $\mathrm{MV}=\% 12.5$

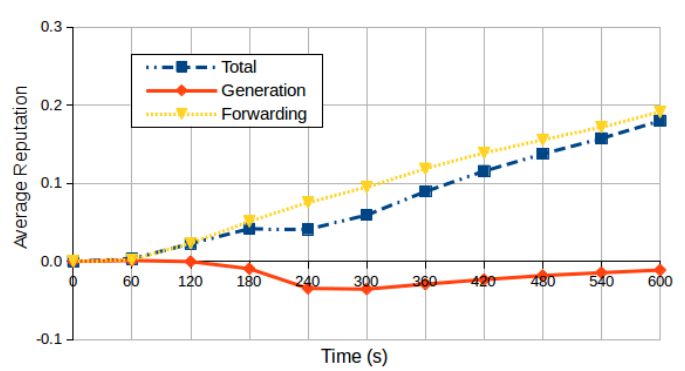

(c) $\mathrm{MV}=\% 50$

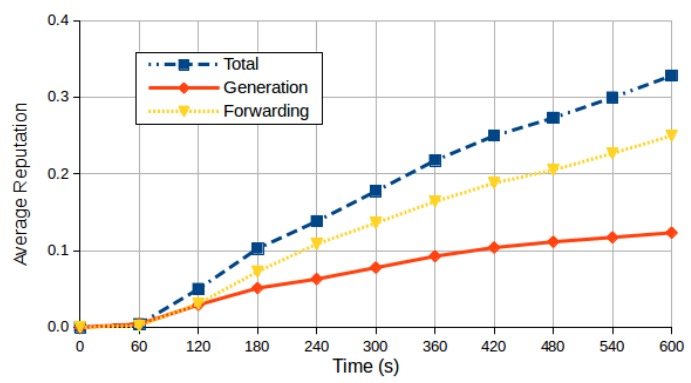

(b) $\mathrm{MV}=\% 25$

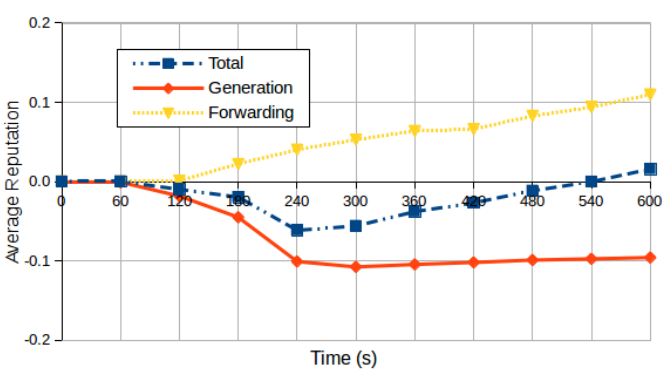

(d) $\mathrm{MV}=\% 75$

Source: Elaborated by the author. 
Figure 28a shows the results of $A R$ for $12.5 \%$ of the $M V$ which are similar to the results of $A R$ for $25 \%$ of the $M V$ in Figure 28b. Results of $A R$ for the generation and forwarding of messages are positive. The results for the generation of messages can be attributed to the fact that the number of vehicles sending fake messages is lower than the number of vehicles sending honest messages, and hence few negative feedbacks were created.

The results of $A R$ for $50 \%$ of the $M V$ are shown in Figure $28 \mathrm{c}$. The results for the generation of messages are negatives because the number of vehicles generating fake messages increases, and thus the number of negative feedback also increases. The results for the forwarding of messages were positive and higher than the results of the total $A R$.

Figure $28 \mathrm{~d}$ depicts the results for $75 \%$ of the $M V$. The influence of the percentage of the $M V$ on the results is more evident in this scenario. The results of the total $A R$ are negatives as are also the results for the generation of messages, and the results by forwarding of messages are the lowest with regard to the other scenarios. It is only in the 540s and 600s range that the total $A R$ increases a little more than zero. The main reason for this is that only 5 vehicles sent honest messages. The results for the generation of messages did not fall to -0.5 in this case, because the malicious vehicles could also receive positive feedback for the forwarding of the messages.

\subsubsection{Effects of the weight for the generation of messages on the Average Reputation (AR)}

Figure 29 compares the final results of the $A R$, for the eight experiments in Table13. The total $A R$ depends on the results of the behavioural factors $\left(K_{1} R e p\right.$ for the generation of messages and $K_{2} R e p$ for the forwarding of messages). The results showed that the total $A R$ is higher for the four experiments with $\delta_{k 1}=0.2$ than for the four experiments with $\delta_{k 1}=0.5$. This is due to the fact that the value of the $\delta_{k 1}$ weight influenced the results of $K_{1}$ Rep and $K_{2} R e p$ (see Equation 4.1.). In these experiments the number of vehicles receiving feedback for the forwarding of messages surpasses the number of vehicles receiving feedback for the generation of messages, due to this, the experiments with the lowest $\delta_{k 1}$ obtained the highest results of the total $A R$.

The results are clarified in Figure 30 which shows the total $A R$, and distinguishes for behavioural factor for the scenario with $12.5 \%$ of the $M V$. It was noted that the scores of the $A R$ for the generation of messages in both scenarios are greater than zero, although there are vehicles receiving negative feedback caused by the generation of fake messages, the number of positive feedbacks exceeds the number of negative feedbacks. Thus, the scenario with less $\delta_{k 1}$ weight (i.e., at 0.2 ) obtained the lowest results for $K_{1} R e p$, and the highest results for $K_{2} R e p$.

Table 14 shows the end results of $A R$ for $\delta_{k 1}=0.2$; while Table 15 shows the end results of $A R$ for $\delta_{k 1}=0.5$. It can be seen that with the increasing of the percentage of the $M V$, the total $A R$ for the generation of messages decreases. The reason for this is the increase in the number of vehicles receiving negative feedback as a result of being sent fake messages. In the 
Figure 29 - Average reputation for the vehicles varying $\delta_{k 1}$ and $\delta_{k 2}=1-\delta_{k 1}$.

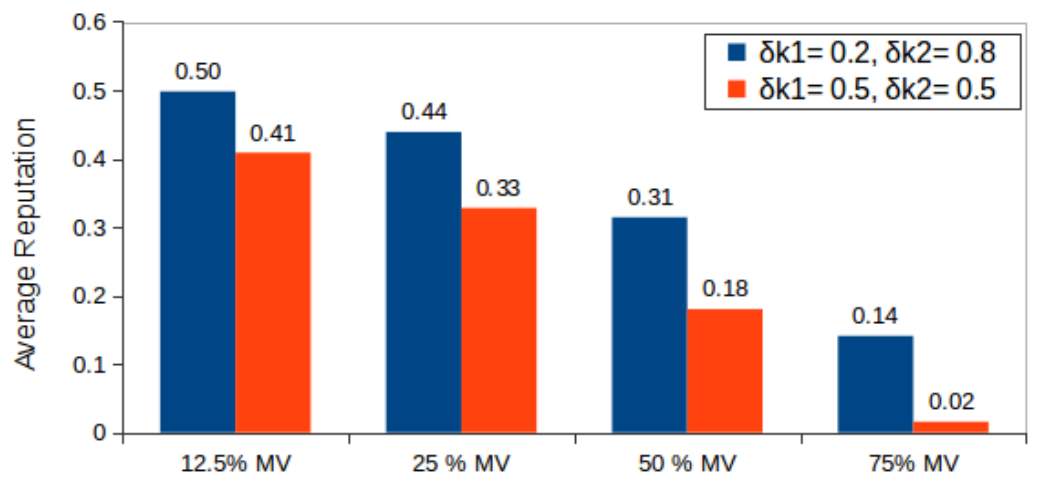

Number of vehicles sending fake messages

Source: Elaborated by the author.

Figure 30 - Average reputation for the scenarios with $12.5 \%$ vehicles generating fake messages varying $\delta_{k 1}$ and $\delta_{k 2}=1-\delta_{k 1}$.

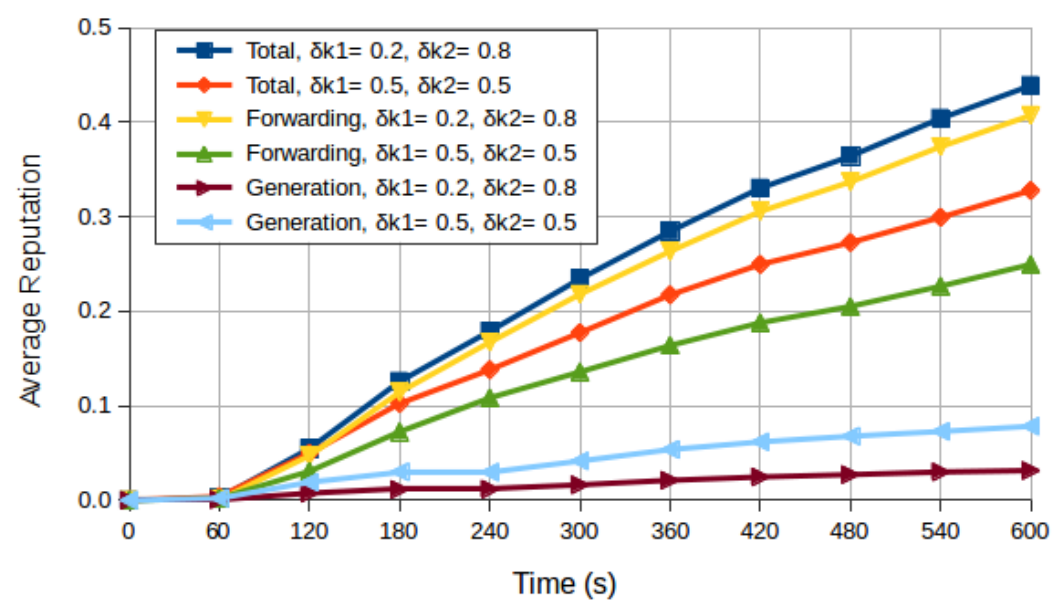

Source: Elaborated by the author.

case of the $75 \%$ of the $M V$ and $50 \%$ of the $M V$, the results of the $A R$ for the generation of messages are negative, because the number of negative feedbacks exceeds the number of positive feedbacks. The lowest result of the total $A R$ is in the scenario with $\delta_{k 1}=0.2$ and $75 \%$ of the $M V$. It is also observed that by increasing of the percentage of the $M V$, the $A R$ for forwarding messages decreases. The reason is that fewer vehicles receive feedback for the forwarding of messages when increases the percentage $M V$.

\subsection{Final considerations}

The performance of PPRS was evaluated in an urban scenario under different application parameters. From the simulation results we concluded that the introduction of geographical areas 
Table 14 - Results of Average Reputation under $\delta_{k 1}=0.2$ and $\delta_{k 2}=0.8$

\begin{tabular}{l|l|l|l}
\hline Scenario & Generation & Forwarding & Total \\
\hline $12.5 \% M V$ & 0.049 & 0.449 & 0.498 \\
$25 \% M V$ & 0.031 & 0.408 & 0.439 \\
$50 \% M V$ & -0.003 & 0.317 & 0.314 \\
$75 \% M V$ & -0.040 & 0.181 & 0.141 \\
\hline
\end{tabular}

Source: Research data.

Table 15 - Results of Average Reputation under $\delta_{k 1}=0.5$ and $\delta_{k 2}=0.5$

\begin{tabular}{l|l|l|l}
\hline Scenario & Generation & Forwarding & Total \\
\hline $12.5 \% M V$ & 0.123 & 0.286 & 0.409 \\
$25 \% M V$ & 0.078 & 0.250 & 0.328 \\
$50 \% M V$ & -0.011 & 0.191 & 0.180 \\
$75 \% M V$ & -0.096 & 0.112 & 0.016 \\
\hline
\end{tabular}

Source: Research data.

of security which enables the checking of reputation score of the neighbouring vehicles reduces the dissemination of fake messages. This reduction is influenced by the opportunistic contact of the vehicles with the RSU, as a means of sending feedback and updating their Reputation Certificates.

We also concluded that the ontological representation of the reputation will help to the server to infer the complex reputation of the vehicles. The reputation score of the vehicles registered by the server reflected their behaviour, e.g., the scenarios where only five vehicles sent fake messages, obtained the highest reputation score. By contrast, the scenarios where 30 vehicles sent fake messages, obtained the lowest reputation score.

Finally, the results revealed that the weights assigned to the behavioural factors for the generation and forwarding of messages influenced the final results of the reputation score of the vehicles. When the weight by the generation of messages increased from 0.2 to 0.5 , the results increased or decreased depending on the number of misbehaving vehicles. Unlike when the weight for forwarding of messages increased from 0.5 to 0.8 , the results increased because there was no negative feedback for this behavioural factor.

The configuration of the reputation system and application parameters is important to avoid, detect and standardise attacks (e.g., the conditions to change from a friendly to an unfriendly area). It was found that a centralised reputation scheme reflects the behaviour of the vehicles insofar as feedbacks are delivered to the Reputation Server. Most of the vehicles were able to update their reputation score from the server through the RSU during the experiments; however it is necessary to improve communications between the vehicles and infrastructure. 
CHAPTER

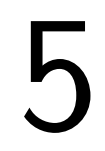

\section{A PSEUDONYM CHANGING STRATEGY BASED ON REPUTATION LEVELS}

In our work, we considered inside intruders with legitimate credentials which are able to trace vehicles by eavesdropping on vehicular communications, and hence violate the privacy in VANETs. For this reason, mechanisms which are generally based on anonymity schemes must be adopted to guarantee the privacy of the vehicle when they authenticate messages. Pseudonym schemes have emerged to meet these privacy and security requirements. In Chapter 3, we proposed a centralised approach called Privacy-Preserving Reputation Scheme (PPRS) for trust management in VANETs; this involved adopting a Public Key Infrastructure (PKI) scheme, in which a Certificate Authority (CA) issues pseudonyms to the vehicles. The CA knows the relationship between the vehicle's real identity and each of its pseudonyms, and the Reputation Server responsible for maintaining the reputation of the vehicles is able to relate anonymous feedbacks to vehicle's real identity. In this chapter, we extend the activity of pseudonym changing of our scheme (see Figure 8) by employing a new strategy. Our strategy dedicated for Vehicle to Vehicle (V2V) communications is based on the assumption that if there are no malicious vehicles around when a change is triggered, there is no risk of tracking and it is not necessary to make changes immediately; the change will go until to exhaust the lifetime of the current pseudonym. Thus, our proposal which is based on a synchronous strategy adds to the change condition the checking of the Reputation Level of the neighbouring vehicles. First, we define the main concepts of our strategy. Second, we provide an overview of the related work on the strategies adopted for pseudonym changing. Third, we propose extending the synchronous pseudonym changing strategy, through a check of the Reputation Level of the neighbouring vehicles. The strategy was evaluated through simulations in a realistic scenario, this include an assessment of the number of used pseudonyms and the successful rate of changed pseudonyms. Fourth, there is a simulation setup and discussion of how the experiments were planned. Finally, the results of the simulations are analysed and some final considerations are made. 


\subsection{Definitions}

If the Finite State Machine of the PPRS in the vehicle receives an event of the change_evt() type, the vehicle adopts a strategy of changing its pseudonym and updates its current pseudonym (see Subsection 3.8). This section defines the concepts that form the building blocks of our strategy of pseudonym changing: privacy, conditional privacy, pseudonyms, pseudonym certificate revocation, traceability problems, the reputation level and information about vehicular status.

\subsubsection{Privacy}

Our scheme provides privacy by means of the properties of unlinkability and untraceability. Unlinkability refers to the fact that the verification of signed messages does not lead to the identification of their senders; and untraceability means that two or more messages generated by the same vehicle are difficult to link to each other. Privacy is guaranteed by pseudonyms which are added to the beacons that are broadcasted periodically by the vehicles.

\subsubsection{Conditional privacy}

Since vehicular information is disseminated by VANETs, conditional privacy preservation must be ensured for accountability processing. Vehicle related privacy information, including the driver's name, licence plate, position, and travelling routes has to be protected, although the legal authorities must be able to reveal this information in case of a serious event, such as a crime or car accident.

Figure 31 - Public Key Infrastructure for VANETs

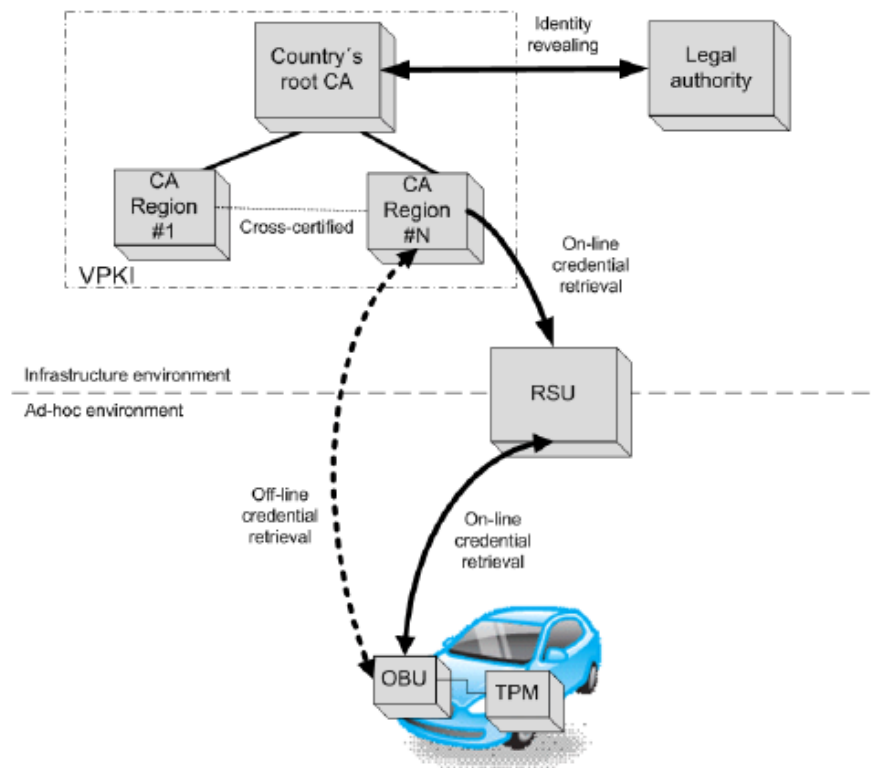

Source: Fuentes, González-Tablas and Ribagorda (2010). 


\subsubsection{Pseudonyms}

Pseudonyms are short-term public key certificates that do not contain any information identifying the vehicle. The PKI scheme involves creating pseudonyms and obtaining their keys from the Certificate Authority, and then keeping this cryptographic information in the Tamper-Proof Device (TPD) or in the Trusted Platform Module (TPM) of the vehicles. The pseudonym refill for the TPD or TPM may be off-line or on-line, (see Figure 31). In the first alternative, the CA sends all the data to the vehicle periodically but this requires too much storage in the vehicle. In the second alternative, the vehicle downloads the data through Roadside Units (RSUs) when required. Table 16 compares the pros and cons of the two options (MA; KARGL; WEBER, 2008).

Table 16 - Comparing pseudonym refill strategies

\begin{tabular}{l|l|l}
\hline Option & off-line & on-line \\
\hline Connectivity to CA & Occasional & Very often \\
\hline Communication overhead & low & slightly higher \\
\hline Pseudonyms to refill & difficult to predict & easy to estimate \\
\hline Pseudonym storage & big storage & very small storage \\
\hline Vulnerability time window & big & very small \\
\hline Pseudonym certificate revocation & no good solution & minimized-not need \\
\hline Deployment cost & low & relatively high \\
\hline
\end{tabular}

Source: Ma, Kargl and Weber (2008).

\subsubsection{Pseudonym certificate revocation}

Sometimes in the face of a continuous misbehaviour by a vehicle, the revocation of a pseudonym is necessary. There are two ways of dealing with this; first, the Certificate Revocation List (CRL) can be used, which must include all the peer's non-expired pseudonyms public keys. The second method is to specify a pseudonym update rate so that the vehicles certificates will quickly run out of. In our implementation, the pseudonyms have an expiration date which determines how often a vehicle updates their pseudonyms.

\subsubsection{Traceability problem}

The use of a single pseudonym is not enough to protect privacy, in particular the property of untraceability. As shown in Figure 32, when three vehicles are travelling on the road, if only one vehicle changes its pseudonym during $\triangle t$, an adversary can still monitor the pseudonym link. Although the three vehicles may change their pseudonyms at the same time, the information about location and velocity embedded in safety messages can still provide a clue about how an adversary is able to link the pseudonyms, and cause the privacy protection to fail. For this reason, it is essential to ensure the location privacy which can be achieved by frequently changing pseudonyms in the vehicles (BERESFORD; STAJANO, 2004; FREUDIGER et al., 2007). 
Figure 32 - Pseudonyms being changed on the wrong occasion

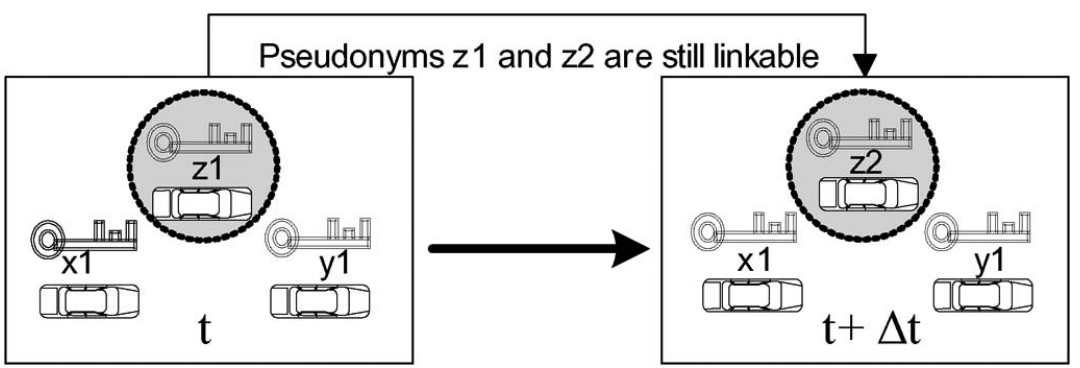

Source: Lu et al. (2012).

To address this problem, each vehicle should make use of multiple pseudonyms, which can be changed frequently from one pseudonym to another. The number of pseudonyms depends on the frequency of the changes which ranges from seconds to hours (ALEXIOU et al., 2013). By increasing the frequency of pseudonyms changing, the chances of an intruder being able to launch a successful attack against privacy will be significantly reduced, but there will be a sharp increase in the amount of storage space needed for saving pseudonyms. Moreover, if the pseudonyms are changed in an incorrect way, the solution is ineffective, since an intruder is still able to link the current pseudonym with the next and its mobility trace can easily be disclosed (BUTTYÁN; HOLCZER; VAJDA, 2007). Simply, changing pseudonyms at an unappropriated time or with an improper status wastes pseudonyms, and leads to an increase in the number of pseudonyms needed for a vehicle, and accordingly, more resources are required for storing or calculating them. In the case of VANETs with a very large number of vehicles, it is a big challenge to handle such a huge number of pseudonyms.

\subsubsection{Reputation Certificate}

The Reputation Certificate (RC) is a certificate issued and signed by the Reputation Server. The RC contains the latest reputation score of the vehicle that is kept and updated by the reputation server on the basis of the feedback supplied by the peer vehicles in the Vehicular Ad hoc Network (VANET), (see Subsection 3.5.6). The RC has an expiration date and the vehicles must frequently update it, to prevent that misbehaving vehicles continue acting in the VANET. The vehicle updates its RC through an opportunistic contact with the Roadside Unit.

\subsubsection{Anonymity}

Anonymity of a vehicle refers when it is not identifiable within the anonymity set. The anonymity set of a vehicle includes the vehicle and its neighbours when they change pseudonyms together at the same place-time. The strategies of pseudonym changing seek to improve this property. A truly anonymous system must remove any anonymity-compromising information, or at least make it less useful to an attacker. 


\subsubsection{Reputation Level}

The term "Reputation Level" is introduced to contrast with "Reputation $\left(\operatorname{Rep}_{v}\right)$ " as defined in Chapter 3 (Subsection 3.5.1). The Reputation Level of a vehicle, denoted as $R L_{v}$, is a discrete approximation of the $\operatorname{Rep}_{v}$ maintained by the Reputation Server (RS) and contained in the Reputation Certificate. An example of mapping the $R e p_{v}$ of 0.2 to $R L_{v}$ would be rounding off the decimal and getting a result of 0 . A backward mapping from $R L_{v}$ to $R e p_{v}$ should be impossible.

In our strategy, the Reputation Level is incorporated in the beacons and is assigned one of two possible values. This is, $R L_{v}$ is assigned the value $\mathbf{+ 1}$ for the vehicles with good reputation ( $R e p_{v}$ greater than zero) and $\mathbf{- 1}$ for the misbehaving vehicles ( $R e p_{v}$ less than or equal to zero). Thus, our strategy protects the anonymity of the vehicles due to that the beacons do not reveal an identifiable reputation level.

\subsubsection{Information about vehicular status}

Each vehicle broadcasts periodically beacon messages openly to all of its neighbours. Beacons include information about vehicular status such as velocity, position and heading direction. This information is used by a pseudonym changing strategy to determine the "change condition", i.e., if two or more vehicles have a similar status. Two vehicles have a similar status if their heading directions are the same, their velocities differ in terms of $v e l ~ m / s$, and the distance between them is less than $d-\min \mathrm{m}$, (LIAO; LI, 2009).

\subsection{Pseudonym Changing Strategies}

In this section, work related to pseudonym changing strategies in VANET is reviewed. In (LI et al., 2006), the pseudonym changes when the vehicle alters its direction and speed. Thus, an adversary cannot make use of the predictability of node movements to correlate node locations before and after an update. In strategies based on density, the vehicle changes its pseudonym in accordance with the current density of neighbouring traffic and thus avoids ineffective pseudonym changes when the vehicle is alone. The authors of (CHAURASIA; VERMA, 2008; CHAURASIA et al., 2009) propose that a vehicle should update its pseudonym when the density is above a fixed threshold. These individual strategies are implemented in a simple way, but they have low anonymity.

Others strategies determine a fixed time (periodic) to change the pseudonym, (ECKHOFF et al., 2010) employs a time-slotted pseudonyms pool. Instead of storing a very large number of pseudonyms, every vehicle keeps a set of pseudonyms (called a pseudonyms pool) which are used for specific time slots. The length of the time slot determines how often a vehicle changes its pseudonym. The benefit of this individual strategy is that a vehicle always has a valid pseudonym 
even if the Certificate Authority is not reachable. However, as soon as the attacker knows the period of pseudonym changing which is easy to discover, tracking becomes possible (ROUFA et al., 2010).

The random change strategy usually generates a random number before broadcasting a beacon. The pseudonym is changed if the random number is below a threshold which is set in advance (PAN et al., 2011). As a result, an adversary cannot predict the next pseudonym changing. However, tracking is still possible if only one or a few vehicles change pseudonyms at a determined time, because all the other neighbours will keep the same identity. Thus, linking the new and old pseudonym of the vehicle that carried out the change, is still feasible.

In the mix-context approach a vehicle changes the pseudonym and then evaluates if it found vehicles nearby with a similar status (as defined in 5.1.9). If the change is not successful, the system tries to change the pseudonym again, and in the worst scenario the system may change pseudonyms continually, which results in more pseudonyms being used (GERLACH, 2006; GERLACH; GUTTLER, 2007). In addition, the simulations only took account of partial information about vehicular status (i.e. the position of the vehicle) to deduce the number of neighbouring vehicles with similar status. The work in (GERLACH; GUTTLER, 2007) includes all the status information as defined in 5.1.9, but different from the work in (GERLACH; GUTTLER, 2007), i.e. after one pseudonym has changed, no matter whether the change of the pseudonym is successful or not. This prevents the system from changing pseudonyms continually, and thus needing more pseudonyms.

In (LI et al., 2006) a vehicle can exchange pseudonyms with one of its neighbours on request. This scheme requires the access point to forward the swap messages. (FREUDIGER et al., 2010) presents an age-based strategy which the neighbours of a vehicle can choose to cooperate with it to change pseudonym depending on their expiration dates, upon receiving the request from the vehicle (FREUDIGER et al., 2010). The request might cause a more asynchronous pseudonym change, and thus weaken the anonymity.

The synchronous strategy incorporates the similar status strategy and inserts a flag into the beacons to indicate if a vehicle is eligible to change its pseudonym, and thus increases the probability of pseudonyms changing simultaneously (LIAO; LI, 2009; LIAO; LI; PAN, 2009; ECKHOFF et al., 2010). In a synchronous strategy, when a vehicle meets the trigger, its neighbours may not meet the trigger as well. The authors of (PAN; LI, 2013) establish a general framework of cooperation for pseudonyms changing, its purpose is to enable to the neighbours to change pseudonyms by utilising the vehicle's trigger, so that all neighbours change pseudonyms together. This mechanism increases of degree of anonymity, but require more effort and control. 


\subsection{Improving the synchronous change strategy using the Reputation Level (RL)}

In VANETs, the reputation can be used as an important standard for making decisions, such as forwarding or rejecting packets sent by a vehicle, regarding or disregarding it as an option in the routing of data, etc (BIDÓIA et al., 2014). Our strategy assumes that if there are no malicious vehicles around when the change condition is triggered, there is no risk of tracking and a change is not necessary; in another way the pseudonym will be wasted. We propose add the information of the Reputation Level $(R L)$ of the neighbour vehicles to the parameters of evaluation of the pseudonym changing, together with the vehicular status information and the synchronisation with other vehicles. Our goal is to reduce the number of pseudonyms wasted, thus it is economised the pseudonyms that a vehicle has and the resources for their storing, that it is critical in the pseudonym refill off-line. Similarly, economising the pseudonyms used in the vehicles will allow to reduce the communication overhead and frequency of refill in the option on-line.

Our proposal is based on the synchronous strategy in (LIAO; LI, 2009) since both simultaneity of changing pseudonyms and vehicular status information are taken into consideration. For synchronisation the change flag is inserted in the beacons which is used to announce that the vehicle is ready to change its pseudonym. As shown in Figure 33, the vehicle uses a current pseudonym for a time of $60 \mathrm{~s}$, then the algorithm enables the change flag in the communications to indicate to the other vehicles that it plans to change the pseudonym. The minimum time was chosen because it represents a reasonable value for position based routing (LOCHERT et al., 2003). After this, the system enters a wait-check sub-cycle where it remains until that the change condition is fulfilled. The change condition involves a minimum number of $K$ neighbour vehicles with the change flag enabled, similar status, and one of them have a negative Reputation Level. If the previous condition is not fulfilled, the vehicle waits for a maximum time of $60 \mathrm{~s}$ to change the pseudonym. In this way, a minimum time of change between two pseudonyms can be guaranteed.

\subsection{Simulation setup}

We simulated three pseudonym-changing strategies to ensure that a more complete comparison could be made for measuring the effectiveness of our strategy: an individual strategy (with a similar status) and two collective strategies (synchronous and including the Reputation Level of the vehicles). The simulation environment was exposed in Section 2.9.1.. 
Figure 33 - Pseudonym changing algorithm

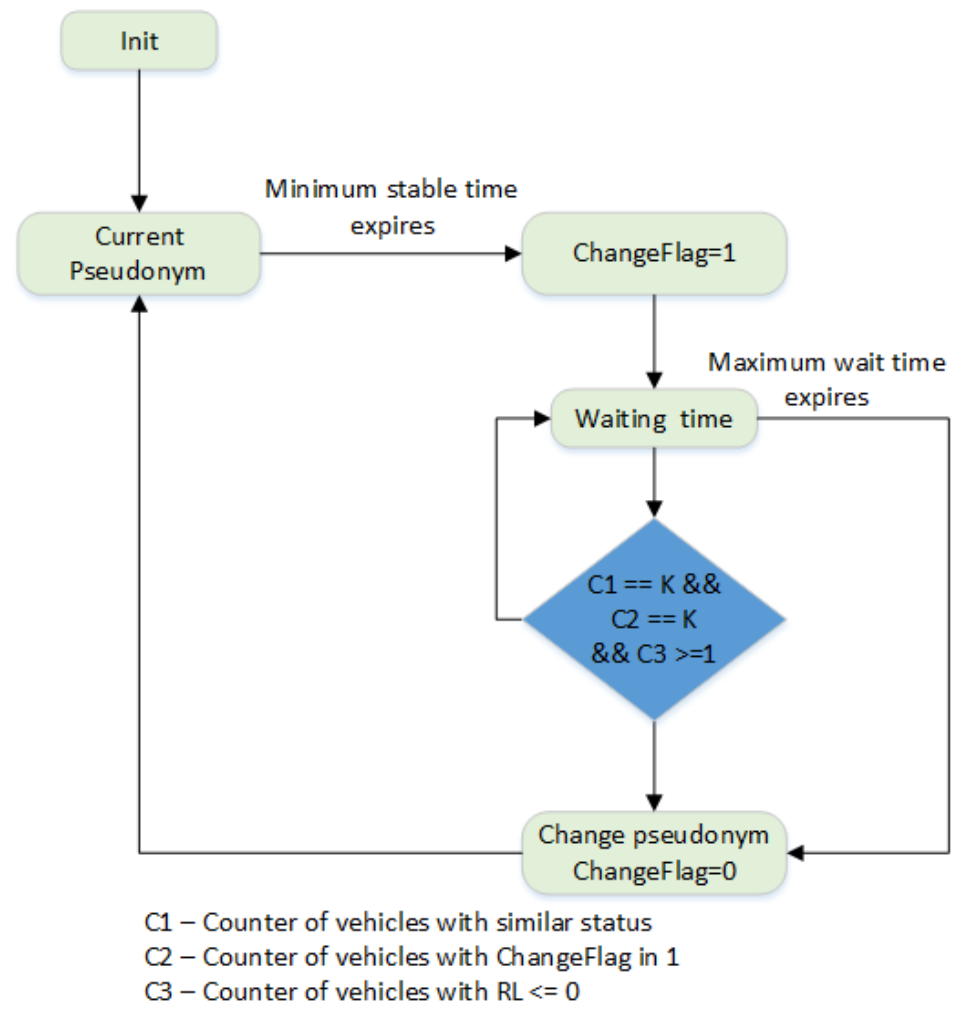

Source: Elaborated by the author.

\subsubsection{Simulation scenario}

A scenario built in the iTETRIS ("An Integrated Wireless and Traffic Platform for Real-Time Road Traffic Management Solutions") project was selected. The given data included representations of the areas around the "Andrea Costa" and the "Pasubio" roads, (see Figure 34). The scenario included the traffic demand for Bologna's peak rush-hour (8:00am - 9:00am) (BIEKER et al., 2015) and the flow of vehicles in the scenario was identified, (see Figure 35). A total of 1843 vehicles enter the scenario but not all them keep into it. The blue line represents the vehicles entering the scenario. The red stands for the vehicles that going out from the simulated scenario. It can be seen that the number of vehicles represented in the blue and red lines maintains an increasing minute by minute, but the number of vehicles going out is lower than the number entering. For these reasons, the yellow line indicating the number of vehicles that stayed in the simulation is constant. It should be noted that about 600 vehicles per minute travel into the areas around the roads of the scenario.

\subsubsection{Simulation parameters}

Table 17 shows the main parameters of mobility and network used in our simulations. We performed our simulations for 600s which 1843 vehicles entered the scenario. 
Figure 34 - Selected views of the "joined" scenario from iTETRIS

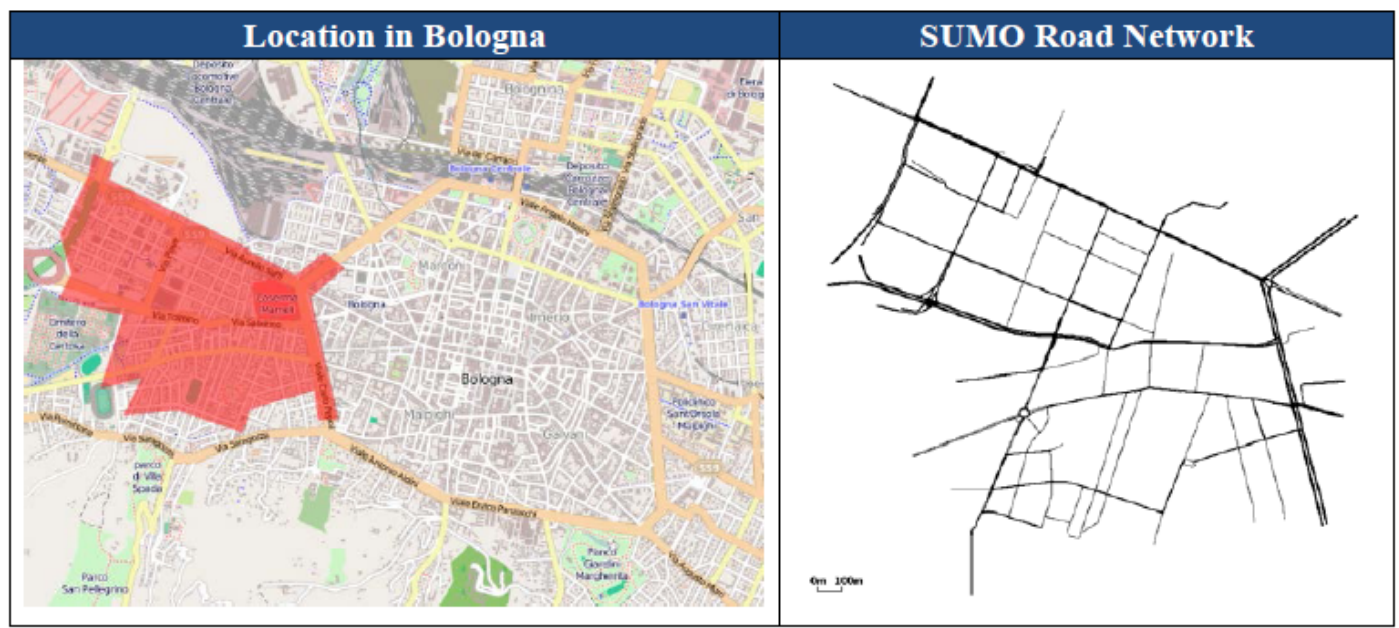

Source: Bieker et al. (2015).

Figure 35 - Number of vehicles in the Bologna scenario.

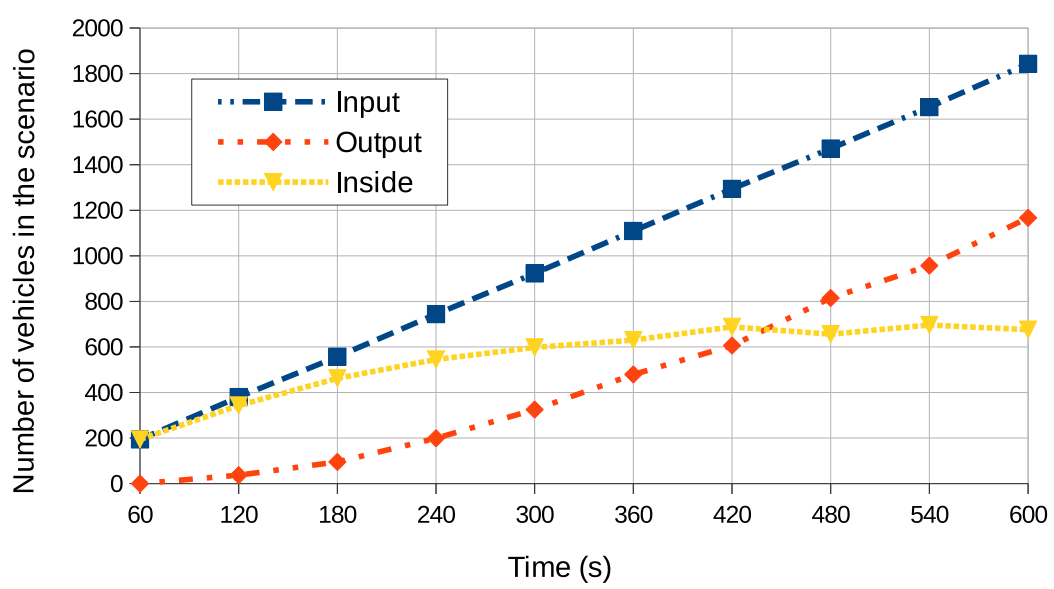

Source: Elaborated by the author.

\subsubsection{Planning the experiments}

We selected a) the metrics of the number of used pseudonyms, b) number of successfully changed pseudonyms and c) successful rate of the changed pseudonym as in the work (LIAO; LI, 2009). We incorporated the metric of average time for pseudonym changing. A Misbehaviour Vehicle $(M V)$ is a vehicle that will always send fake messages, but it will also report the feedback of the peers correctly. The response variables and factors are defined below.

\section{i Metrics}

- Number of used pseudonyms: it refers to the total number of current pseudonyms attached to the vehicles as the result of the changing strategy; 
Table 17 - Mobility, scenario, and network parameters

\begin{tabular}{l|l}
\hline Parameter & Value \\
\hline Maximum velocity & $50 \mathrm{~km} / \mathrm{h}$ \\
Average velocity & $28 \mathrm{~km} / \mathrm{h}$ \\
Urban area & $4.5 \mathrm{Km}^{2}$ \\
Number of vehicles & 1843 \\
Transmission rate & $6 \mathrm{Mbps}$ \\
Communication range & $250 \mathrm{~m}$ \\
MAC protocol & IEEE $802.11 \mathrm{p}$ \\
Network protocol & Wave Short Message Protocol \\
Beacon frequency & $1 \mathrm{~Hz}$ \\
Simulation time & $600 \mathrm{~s}$ \\
\hline
\end{tabular}

Source: Elaborated by the author.

- Number of successfully changed pseudonyms: it refers to the total number of pseudonyms that are changed without risk of being tracked;

- Successful rate of changed pseudonyms: it refers to the total number of successfully changed pseudonyms with regard to the number of used pseudonyms;

- Average time for pseudonym changing: this refers to the average maximum waiting time for the vehicles during the change of the pseudonym;

ii Fixed Factors: the first three factors, as configured in (LIAO; LI, 2009)

- vel: it is the difference in speed between two vehicles defined for the status strategy, for our implementation was defined in $0.5 \mathrm{~m} / \mathrm{s}$;

- $d-\min$ : it is the distance between two vehicles defined for the status strategy, for our implementation it was defined as $20 \mathrm{~m}$;

- $K$ : number of neighbours in the synchronous strategy with the change flag enabled, for our implementation this was defined as 2;

- Elliptic curve: it refers to the type of elliptic curve used to implement the functions of signing and verification of the pseudonyms; we selected the secp256r curve.

iii Variable Factors

- Penetration Rate $(P R)$ : this refers to the ratio of the number of vehicles installed with communication modules to the total number of vehicles in the simulation. The VANETs will grow gradually until all the vehicles are fitted with wireless technology;

- Pseudonym-changing strategy:

- Status: it strategy checks if the vehicles have a similar status, as defined in Subsection 5.1.9; 
- Synchronous: in this strategy, both the simultaneity and vehicular status information are taken into account for pseudonym changing. Simultaneity is achieved when a number $K$ of neighbouring vehicles enable the change flag, (LIAO; LI, 2009);

- Including the Reputation Level: in this strategy, the simultaneity, vehicular status information and the Reputation Level are taken into account for pseudonym changing, as defined in Section 5.3;

- Percentage of Malicious Vehicles (MV): this is the number of vehicles that have a negative Reputation Level with regard to the number of vehicles in the simulation.

A total number of 20 experiments were carried out and the Penetration Rate $P R$ ranged from $40 \%$ to $100 \%$ during the strategy of pseudonym changing. Three cases were conducted for the strategy including the Reputation Level: 50\%, 10\% and 0\% of $M V$. Table 18 shows the planned experiments.

Table 18 - Planning of experiments to evaluate the impact of the strategies of pseudonyms changing

\begin{tabular}{l|l|l}
\hline Experiment & PR & Strategy \\
\hline 1 & $100 \%$ & Status \\
2 & $100 \%$ & Synchronous \\
3 & $100 \%$ & RL with 0\% $M V$ \\
4 & $100 \%$ & RL with 10\% MV \\
5 & $100 \%$ & RL with 50\% MV \\
6 & $80 \%$ & Status \\
7 & $80 \%$ & Synchronous \\
8 & $80 \%$ & RL with 0\% $M V$ \\
9 & $80 \%$ & RL with 10\% $M V$ \\
10 & $80 \%$ & RL with 50\% $M V$ \\
11 & $60 \%$ & Status \\
12 & $60 \%$ & Synchronous \\
13 & $60 \%$ & RL with 0\% $M V$ \\
14 & $60 \%$ & RL with 10\% $M V$ \\
15 & $60 \%$ & RL with 50\% $M V$ \\
16 & $40 \%$ & Status \\
17 & $40 \%$ & Synchronous \\
18 & $40 \%$ & RL with 0\% $M V$ \\
19 & $40 \%$ & RL with 10\% $M V$ \\
20 & $40 \%$ & RL with 50\% $M V$ \\
\hline
\end{tabular}

Source: Research data. 


\subsection{Results and discussion}

The metrics selected to compare the results are as follows a) the number of used pseudonyms, b) the number of successfully changed pseudonyms, c) the successful rate of changed pseudonyms, and d) the average time needed for the pseudonym changing.

\subsubsection{Effect of the strategies on the number of used pseudonyms}

Figure 36 compares the total number of used pseudonyms for the 1,2,3 and 4 experiments in Table 18. The experiments assumed a penetration rate of $100 \%$ and the four strategies were: status, synchronous strategy, reputation level with $0 \% M V$ and reputation level with $10 \% M V$. It should be noted that the status strategy obtained the highest number of used pseudonyms because this strategy is able to find the conditions needed to change the pseudonym more quickly. In contrast, in the case of the strategy of RL with $0 \% M V$ where there was an absence of misbehaving vehicles, the strategy did not change the pseudonyms until the maximum waiting time had expired, as we expected. The results for the reputation strategy with $10 \%$ of $M V$ were close to the results of the synchronous strategy. The main reason is that the presence of only $10 \%$ of misbehaving vehicles influenced to that the strategies will find similar conditions for pseudonym changing.

Figure 36 - Comparison among strategies on the total number of used pseudonyms

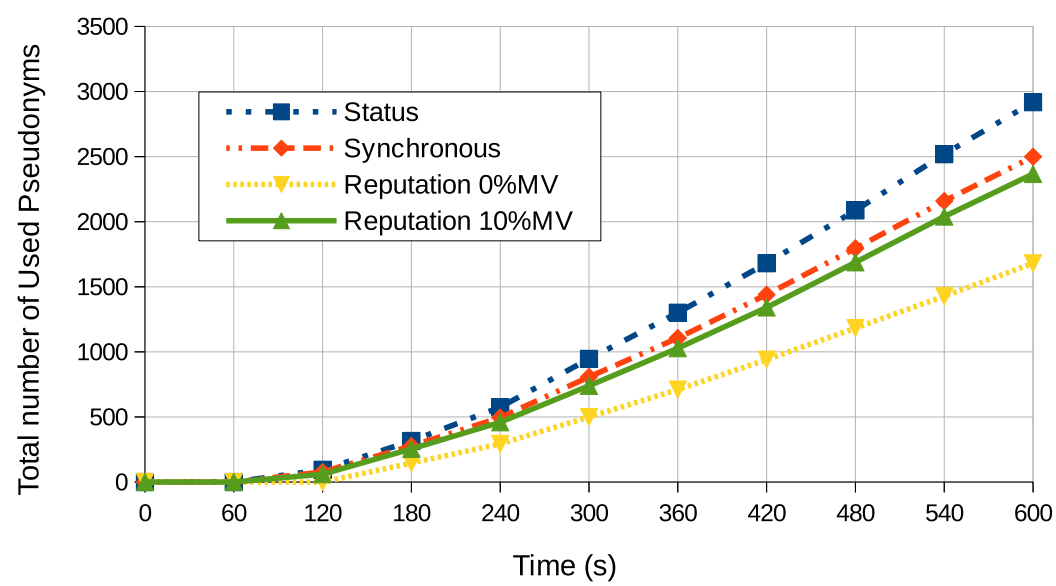

Source: Elaborated by the author.

\subsubsection{Effect of the strategies on the number of successfully changed pseudonyms}

Figure 37 compares the total number of successfully changed pseudonyms with the 1,2,3 and 4 experiments in Table 18. The experiments assumed a penetration rate of $100 \%$ and four strategies: status, synchronous strategy, reputation level with $0 \% M V$ and reputation level with 
$10 \% M V$. The status strategy had the lowest anonymity because the probability of changing pseudonyms simultaneously with other neighbouring vehicles was low. On the other hand, this probability increases with the synchronous strategy where the results with successfully changed pseudonyms were the highest. The results for the case with $10 \% M V$ are close to the results of the synchronous strategy due to the similarity of the condition of pseudonym changing. The results for the case with $0 \% M V$ were higher than the results of the status strategy, and lower than the results of the synchronous strategy.

Figure 37 - Comparison among strategies on the total number of successfully changed pseudonyms

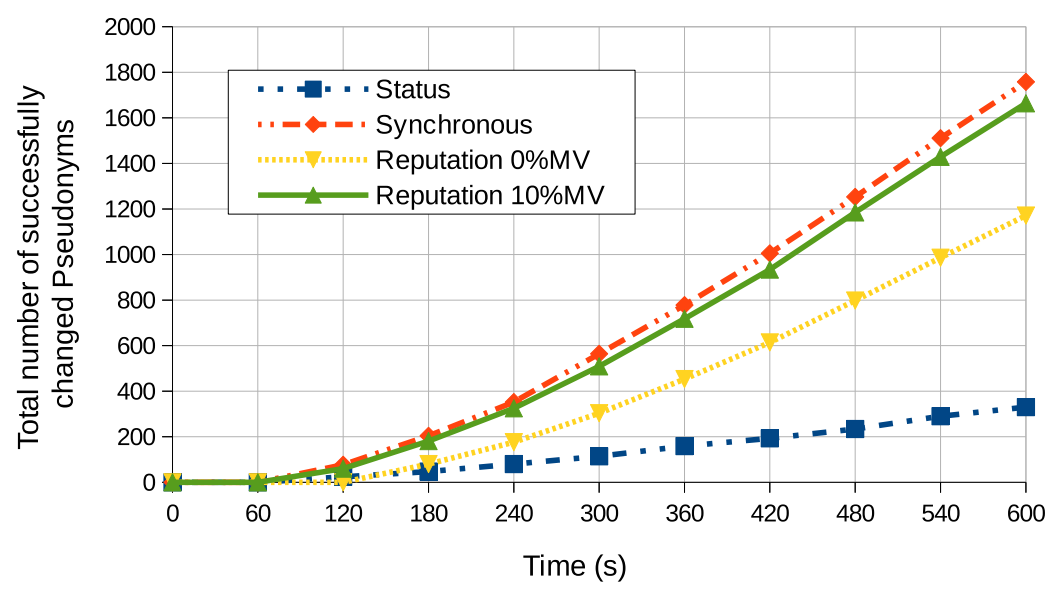

Source: Elaborated by the author.

\subsubsection{Effect of the strategies on the average time for pseudonym changes}

Figure 38 depicts the results of the average time to change pseudonyms for the 1,2,3,4 and 5 experiments in Table 18. The average time is higher for the reputation level strategy (with $0 \% M V$ ) than for the other strategies, the time for pseudonym changing goes on until the maximum waiting time, i.e., 120s. This is because all vehicles had a good reputation and therefore were not made changes of pseudonyms before of the 120s. Instead, when the reputation level strategy has $10 \% M V$ or $50 \% M V$, the average time decreases because some neighbouring vehicles have bad reputation and the strategy changes of pseudonym with more frequency. In the strategy of reputation level, if the percentage of $M V$ increases, the average time in the reputation level strategy will be close of the results of the synchronous strategy because the number of pseudonyms used was very similar in the two strategies, as shown in Figure36. 
Figure 38 - Average time for pseudonym changing

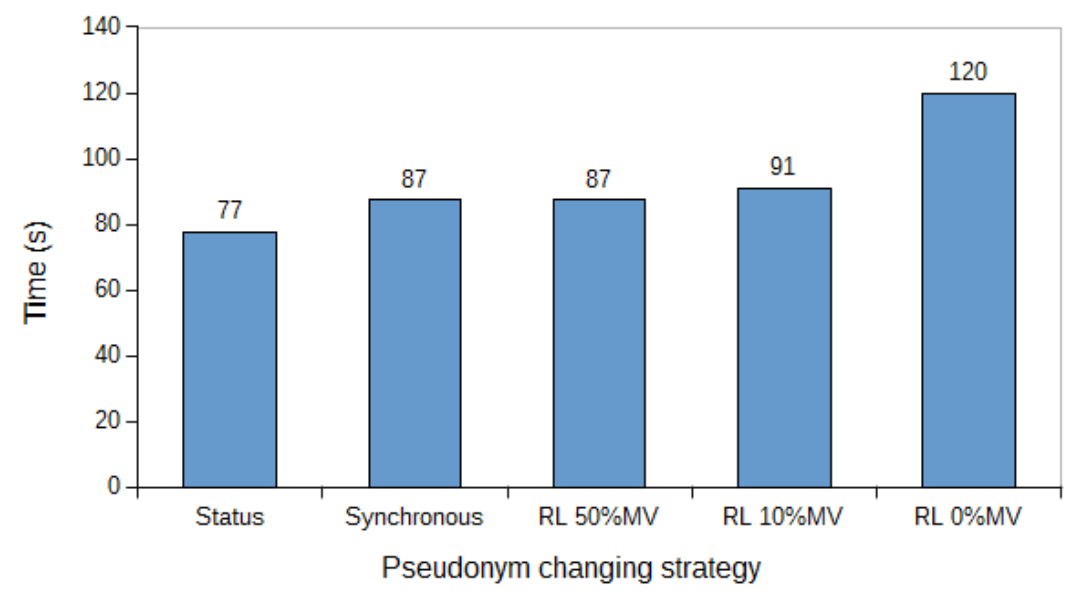

Source: Elaborated by the author.

Figure 39 - Successful rate of changed pseudonyms

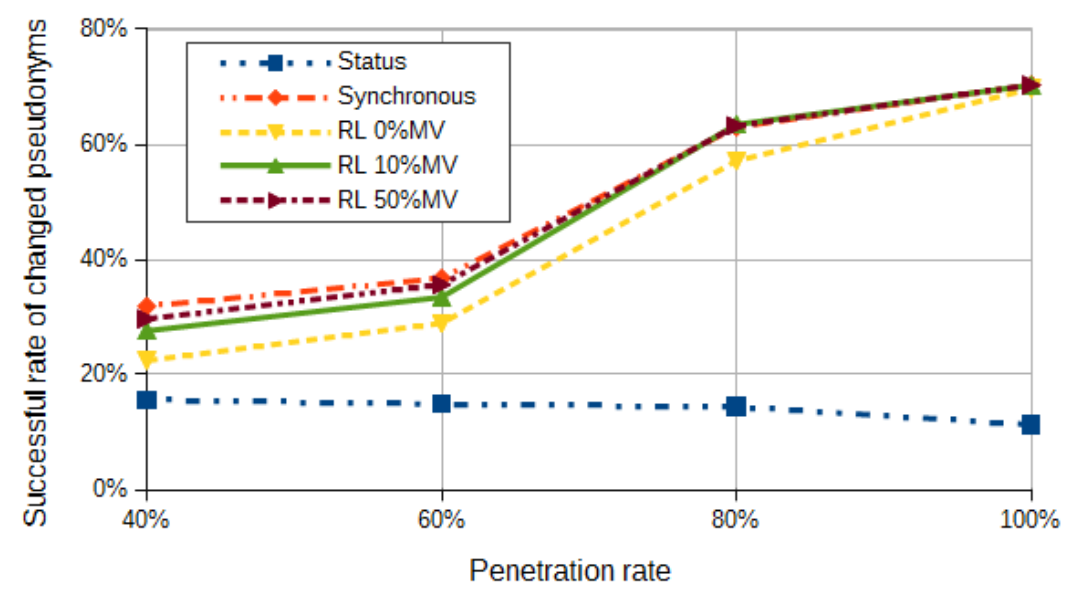

Source: Elaborated by the author.

\subsubsection{Effect of the strategies on the successful rate of changing pseudonyms}

Figure 39 shows the results of successful rate of changing pseudonyms for all the experiments in Table 18. It was found that the successful rate of changing pseudonyms for the strategies of reputation and synchronous increases as the Penetration Rate $(P R)$ increases, because to that with more vehicles participating in the system the probability of changing pseudonyms simultaneously with other neighbouring vehicles also increases. In a dense scenario, such as that used in our simulations, even in low penetration rate (40\%), the results of the successful rate of pseudonym changing for the status strategy is lower that for the other strategies. This is because, in the status strategy, the probability that at least two vehicles with similar status changes their pseudonym is lower. It was noted that with the $100 \%$ of $P R$, the results of successful rates for the 
strategies of reputation and synchronous were similar, about 70\%. In the reputation level strategy, if the percentage of $M V$ increases, the successful rate in the reputation level strategy will be close of the results of the synchronous strategy because the number of successfully changed pseudonyms for both strategies was very similar which is shown in Figure 37.

\subsection{Final considerations}

The implementation of pseudonyms in VANETs requires effective and efficient strategies for pseudonym changing to optimise the resources of the vehicle at the same time so that the privacy is not violated. Different strategies have been proposed in the literature; however, our work is innovative in that employs the reputation information of the vehicle which is included in the Reputation Certificate and which is discretised in one of two Reputation Levels. The Reputation Level is added to the beacons broadcasted periodically. This Reputation Level is additional input information for the pseudonym-changing algorithm, that can allow the neighbouring vehicles to make a decision of whether to change a pseudonym when the vehicular status condition and the synchronisation with other vehicles are fulfilled. As the Reputation Level is a discrete value, it does not put the privacy of the vehicles at risk.

We evaluated our proposal in a realistic traffic simulation scenario in the city of Bologna, using the security standards recommended, i.e., digital signatures based on Elliptic Curve Cryptography (ECC). Our results showed that the number of pseudonyms used in the reputation strategy is lower than in the other two schemes, and thus our strategy optimises the use of pseudonyms. If there is an absence of misbehaving vehicles, the pseudonym-changing can go on until the maximum waiting time. When a misbehaving vehicle is detected and the conditions of the trigger are fulfilled, the pseudonym is changed. The results of success rate the change of pseudonym were higher than with the individual strategy and were lower than the synchronous strategy. However, the results without misbehaving vehicles were close to the results of the synchronous strategy. 

CHAPTER

6

\section{CONCLUSIONS AND FUTURE WORK}

\subsection{Conclusion}

The project accomplished the proposed objectives as follows:

- We provided a balanced solution that covered the requirements of security, privacy and trust management in VANETs through the modelled and implementation of the PreservingPrivacy Reputation Scheme (PPRS) for VANETs in which with V2V anonymous communications, it was achieved to lead the historic reputation of the vehicles in the reputation server. Our centralised scheme in comparison with the related work has the advantage of being able to adapt the security state of the system, according to the risk of threat in the VANET defining the geographical areas of security friendly and unfriendly whose purpose is to enable or disable the verification of the reputation of the neighbouring vehicles. Our scheme also makes possible to calculate and update complex reputations, based on more than one behavioural factor, e.g., creation and forwarding of messages. Thus, the reputation of a vehicle can be so much complex as VANET application requires.

- PPRS was evaluated in an application for opportunistic forwarding of messages in which the vehicles got to establish trust in the VANET that it was obtained through feedback for the generation and forwarding of messages. The reputation server received the feedback and updated the global reputation of the vehicles. Thus, our centralised server recorded the behaviour of honest vehicles that sent true messages, misbehaving vehicles that sent fake messages, and altruistic vehicles that participated in the forwarding of messages.

- We provided privacy by means of pseudonyms and analysed the strategies of pseudonym changing to protect the property of untraceability. As a result, the Reputation Level was included as an additional parameter to the synchronous strategy for making a decision to change a pseudonym. Our proposal was evaluated in a realistic traffic simulation scenario 
of Bologna City. Our results showed that the number of pseudonyms used in our strategy is lower than in other schemes, and the success rate obtained from changing the pseudonym was close to the results in the synchronous strategy. It was confirmed that in VANETs the implementation of a good pseudonym changing strategy is important to avoid tracking and optimise the use of pseudonyms.

While studies in the area only mention the need to incorporate privacy mechanisms in VANETs, we create and implement a security platform based on Elliptic Curve Cryptography as recommended by the security standards for the digital signature and pseudonym mechanisms. On the basis of our experiments, we were able to calculate the computational overhead in terms of delay (in ms) employed for signing and checking messages using a processor that is within of the range of those used in mobile devices nowadays. The elliptic curve selected to implement digital signatures in VANETs communications influences the results of delay; an elliptic curve with a larger public key size is subject to higher delays than an elliptic curve with smaller public key size. The processor also affects the results of delay; processors with a higher speed experience lower delays than processors with a lower speed. These results are important to ensure that vehicles can be prepared with better computational resources that reduce delays by signing and checking messages, and thus the vehicles will be able to handle more messages per second.

We evaluate the reputation of the vehicles with regard to the opportunistic forwarding of messages, in which the vehicles received feedback for the behavioural factors of creation and forwarding of messages. We evaluate the performance of PPRS in an urban scenario by means of different parameters, i.e., the weight of the last rating and the weights of the behavioural factors. The attacks involved misbehaving vehicles that generated fake messages in the network during the simulations. The results revealed that the reputation score of vehicles is influenced by the weights attributed to the factors of creation and forwarding of messages, and the weight attributed to the most recent rating. This last weight influenced the number of feedbacks necessary for a vehicle to reach either the maximum or minimum reputation score established for each behavioural factor. The results also showed that the reputation score of the vehicles reflects their behaviour, e.g., the scenario where only $12.5 \%$ of the vehicles send fake messages, obtained the highest reputation score (i.e., 0.45). By contrast, scenarios in which $75 \%$ of the vehicles sent fake messages obtained the lowest reputation score.

It was concluded from the simulation results that checking the reputation score of neighbouring vehicles would reduce the dissemination of fake messages. We noted that PPRS reduced the arrival of fake messages to the destination by $19 \%$ to $30 \%$. These results may seem insignificant, but in conditions of safety applications, a reduction of this kind of messages prevents accidents or saves people's lives. These percentages are influenced by the opportunistic contact of the vehicles with the RSU, which allows them to send feedback and update their reputation certificates. There is an imminent risk that misbehaving vehicles not update their reputation certificates and hence the system fails to filter the fake messages. To overcome 
this problem, the system should set a maximum time for the vehicles to update the reputation certificate, and those vehicles without updated certificates should be given a low reputation by the neighbouring vehicles.

Another issue that influences the results of the reputation of the vehicles, is the reception in the vehicles of the message coming from the server and disseminated by the RSU announcing the change of geographical area between friendly or unfriendly. If the classification of the area is not updated for a vehicle, this will continue without to check the reputation score of their peers and thus fake messages will continue to be forwarded in the network. Despite the problems raised in the above analysis, our server was able to record the behaviour of the vehicles, and show how the misbehaving vehicles obtained a lower reputation than the honest vehicles. When there was more misbehaving vehicles, more negative feedback was provided. We found that a centralised reputation scheme reflects the behaviour of the vehicles insofar as feedbacks are delivered to the reputation server. In our simulations, most of the vehicles were able to update their reputation certificate from the server through a RSU.

Finally, it can be concluded that the VANET applications developed for autonomous vehicles would benefit from our reputation scheme by making decisions based on the evaluation of reputation of neighbouring vehicles. Similarly, the vehicles would benefit from our strategy of pseudonym changing by reducing the number of pseudonyms wasted and economising their resources independently of the method of pseudonym refill chosen. Stakeholders would benefit from the reputation scheme to provide new services and business.

\subsection{Limitations of the study}

Our study did not cover all the measures that should be taken against all kind of attacks although our study examines the main recommendations of the security standards and covers some threats against the reputation system.

\subsection{Future work}

The issues explored in this dissertation pave the way to several new opportunities and raise a number of challenges.

- One important question is how to improve the deduction and the dissemination of the classification of the geographical area to the vehicles?. To address this, we are attempting to design an area discovery protocol.

- A significant work could be to create a complete ontology of reputation that addresses the question of communications and interoperability between the entities involved in VANETs. This could help in the inference of the security state and reputation evaluation; 
the extension could include different types of messages, behavioural factors, vehicle classification, routes, paths, etc. For example, the trust in a neighbouring vehicle could be determined by behavioural factors and different types of messages.

- The parameters and policies with regard to punishment, gain, and initial reputation are important building blocks in any reputation system. A supplementary study will include more details and experiments about this.

- To develop policies for updating certificates and optimise the dissemination of the classification of the geographical area will help to improve the efficiency of the system.

- In future work, we also intend to explore the possibility of extending the evaluation of our scheme to a large-scale by taking account of different kinds of attacks and more performance metrics.

- Finally, the security parameters could also be more dynamic depending on the degree of risk in the VANET. For example, the vehicle could have a set of pseudonyms for two or three categories of elliptic curves with different key size and employ a pseudonym category according to the geographical area where it is.

\subsection{Publications}

i Ullah, K., Santos, L., Michelin, J., dos Santos Moreira, E.: File Transfer in Vehicular Ad-Hoc Networks. In: Computing Systems Engineering (SBESC), 2013 III Brazilian Symposium on. pp. 175-176 (2013).

ii Yokoyama, R. S., Kimura, B. Y., Jaimes, L. M., Moreira, E.D.: A beaconing based opportunistic service discovery protocol for vehicular networks. In Advanced Information Networking and Applications Workshops (WAINA), 2014 28th International Conference. pp. 498-503 (2014)

iii Ullah, K., Santos, L., Yokoyama, R., dos Santos Moreira, E.: Advertising Roadside Services using Vehicular Ad hoc Network (VANET) Opportunistic Capabilities. In: VEHICULAR, 2015 4th International Conference on Advances in Vehicular Systems, Technologies and Applications. pp. 7-13 (2015).

iv Vanni, R. M., Jaimes, L. M. S., Mapp, G., Moreira, E: Ontology Driven Reputation Model for VANET. In AICT 2016, The Twelfth Advanced International Conference on Telecommunications. pp. 14-19 (2016).

v Ullah, K., Santos, L.M., Ribeiro, J.B., Moreira, E.D.S.: SADP: A Lightweight BeaconingBased Commercial Services Advertisement Protocol for Vehicular Ad Hoc Network, pp. 279-293. Springer International Publishing, (2016). 
vi Santos, L.M., Ullah, K., Moreira, E.D.S.: Secure Architecture for Commercial Ads Dissemination on Vehicular Network. Published in 8th IEEE Latin-American Conference on Communications, Nov, 2016.

vii Santos, L.M., Ullah, K., Moreira, E.D.S.: ARS: Anonymous Reputation System on Vehicular Ad-hoc Networks. Published in 8th IEEE Latin-American Conference on Communications, Nov, 2016.

viii Santos, L.M., Moreira, E.D.S.: Privacy-Preserving Reputation Scheme for Vehicular Ad hoc Networks. Submitted to the IEEE Transactions on Mobile Computing Journal, Jun, 2017.

ix Santos, L.M., Moreira, E.D.S.: A Pseudonym changing strategy based on reputation levels. Submitted to the EURASIP Journal on Wireless Communications and Networking, Sep, 2017. 

BIBLIOGRAPHY

AL-KAHTANI, M. S. Survey on security attacks in vehicular ad hoc networks (vanets). In: IEEE. Signal Processing and Communication Systems (ICSPCS), 2012 6th International Conference on. [S.1.], 2012. p. 1-9. Citations on pages 44, 45, 46, and 47.

ALEXIOU, N.; LAGANÀ, M.; GISDAKIS, S.; KHODAEI, M.; PAPADIMITRATOS, P. Vespa: Vehicular security and privacy-preserving architecture. In: ACM. Proceedings of the 2nd ACM workshop on Hot topics on wireless network security and privacy. [S.1.], 2013. p. 19-24. Citation on page 102.

ARIB. Dedicated short-range communication system. ARIB Std T75, p. 1-461, October 2001. Citation on page 40 .

BEHRISCH, M.; BIEKER, L.; ERDMANN, J.; KRAJZEWICZ, D. Sumo-simulation of urban mobility: an overview. In: THINKMIND. Proceedings of SIMUL 2011, The Third International Conference on Advances in System Simulation. [S.1.], 2011. Citation on page 50.

BERESFORD, A. R.; STAJANO, F. Mix zones: User privacy in location-aware services. In: PerCom Workshops. [S.1.: s.n.], 2004. p. 127-131. Citation on page 101.

BIDÓIA, M. C.; CAVENAGHI, M. A.; SPOLON, R.; SPOLON, R.; JR, A. M.; LOBATO, D. C. Simulation of a centralized reputation system for vanets. In: THE STEERING COMMITTEE OF THE WORLD CONGRESS IN COMPUTER SCIENCE, COMPUTER ENGINEERING AND APPLIED COMPUTING (WORLDCOMP). Proceedings of the International Conference on Parallel and Distributed Processing Techniques and Applications (PDPTA). [S.1.], 2014. p. 1. Citation on page 105.

BIEKER, L.; KRAJZEWICZ, D.; MORRA, A.; MICHELACCI, C.; CARTOLANO, F. Traffic simulation for all: a real world traffic scenario from the city of bologna. In: Modeling Mobility with Open Data. [S.1.]: Springer, 2015. p. 47-60. Citations on pages 106 and 107.

BONEH, D.; BOYEN, X.; SHACHAM, H. Short group signatures. In: SPRINGER. Crypto. [S.1.], 2004. v. 3152, p. 41-55. Citation on page 56.

BUTTYÁN, L.; HOLCZER, T.; VAJDA, I. On the effectiveness of changing pseudonyms to provide location privacy in vanets. In: SPRINGER. European Workshop on Security in Ad-hoc and Sensor Networks. [S.1.], 2007. p. 129-141. Citation on page 102.

CALAFATE, C. T.; FORTINO, G.; FRITSCH, S.; MONTEIRO, J.; CANO, J.-C.; MANZONI, P. An efficient and robust content delivery solution for ieee $802.11 \mathrm{p}$ vehicular environments. Journal of Network and Computer Applications, Elsevier, v. 35, n. 2, p. 753-762, 2012. Citation on page 37.

CAO, Z.; LI, Q.; LIM, H. W.; ZHANG, J. A multi-hop reputation announcement scheme for vanets. In: IEEE. Service Operations and Logistics, and Informatics (SOLI), 2014 IEEE International Conference on. [S.1.], 2014. p. 238-243. Citation on page 56. 
CHAURASIA, B. K.; VERMA, S. Optimizing pseudonym updation for anonymity in vanets. In: IEEE. Asia-Pacific Services Computing Conference, 2008. APSCC'08. IEEE. [S.1.], 2008. p. 1633-1637. Citation on page 103.

Trust based group formation in vanet. International Journal of Modern Traffic and Transportation Engineering Research (MTTER), v. 2, n. 2, p. 121-125, 2013. Citations on pages 28,43 , and 57 .

CHAURASIA, B. K.; VERMA, S.; TOMAR, G. S.; BHASKAR, S. Pseudonym based mechanism for sustaining privacy in vanets. In: IEEE. Computational Intelligence, Communication Systems and Networks, 2009. CICSYN'09. First International Conference on. [S.1.], 2009. p. 420-425. Citation on page 103.

CHEN, L.; LI, Q.; MARTIN, K. M.; NG, S.-L. A privacy-aware reputation-based announcement scheme for vanets. In: WiVeC. [S.1.: s.n.], 2013. p. 1-5. Citations on pages 56 and 57.

Private reputation retrieval in public-a privacy-aware announcement scheme for vanets. arXiv preprint arXiv:1506.03588, 2015. Citations on pages 56 and 57.

CHEN, Y.-M.; WEI, Y.-C. A beacon-based trust management system for enhancing user centric location privacy in vanets. Journal of Communications and Networks, IEEE, v. 15, n. 2, p. 153-163, 2013. Citation on page 58.

CHENG, H. T.; SHAN, H.; ZHUANG, W. Infotainment and road safety service support in vehicular networking: From a communication perspective. Mechanical Systems and Signal Processing, Elsevier, v. 25, n. 6, p. 2020-2038, 2011. Citation on page 47.

CHRISTIN, D.; ROSSKOPF, C.; HOLLICK, M.; MARTUCCI, L. A.; KANHERE, S. S. Incognisense: An anonymity-preserving reputation framework for participatory sensing applications. Pervasive and mobile Computing, Elsevier, v. 9, n. 3, p. 353-371, 2013. Citations on pages 61 and 79.

Dan Brickley and Libby Miller. FOAF Vocabulary Specification 0.99. 2014. Available: <http: //xmlns.com/foaf/spec/>. Accessed: 30/03/2017. Citation on page 58.

DELLAROCAS, C. Immunizing online reputation reporting systems against unfair ratings and discriminatory behavior. In: ACM. Proceedings of the 2nd ACM conference on Electronic commerce. [S.1.], 2000. p. 150-157. Citation on page 48.

DHAMGAYE, A.; CHAVHAN, N. Survey on security challenges in vanet 1. Citeseer, 2013. Citations on pages 42, 45, 46, and 47.

DING, Q.; LI, X.; JIANG, M.; ZHOU, X. Reputation management in vehicular ad hoc networks. In: IEEE. Multimedia Technology (ICMT), 2010 International Conference on. [S.1.], 2010. p. 1-5. Citation on page 89.

DOMINGO-FERRER, J.; WU, Q. Safety and privacy in vehicular communications. In: Privacy in Location-Based Applications. [S.1.]: Springer, 2009. p. 173-189. Citations on pages 33 and 35 .

ECKHOFF, D.; SOMMER, C.; GANSEN, T.; GERMAN, R.; DRESSLER, F. Strong and affordable location privacy in vanets: Identity diffusion using time-slots and swapping. In: IEEE. Vehicular Networking Conference (VNC), 2010 IEEE. [S.1.], 2010. p. 174-181. Citations on pages 103 and 104. 
ENGOULOU, R. G.; BELLAÏCHE, M.; PIERRE, S.; QUINTERO, A. Vanet security surveys. Computer Communications, Elsevier, v. 44, p. 1-13, 2014. Citation on page 69.

ETSI. Part 1: Technical characteristics for pan-european harmonized communications equipment operating in the $5 \mathrm{ghz}$ frequency range and intended for critical road-safety applications; system reference document. ETSI TR 102 492-1, p. 1-31, June 2005. Citation on page 37.

ETSI. Intelligent transport systems (ITS); european profile standard for the physical and medium access control layer of intelligent transport systems operating in the $5 \mathrm{ghz}$ frequency band. ETSI ES 202663 V1.1.0, p. 1-44, November 2009. Citation on page 40.

ETSI. ETSI TS 102941 V1.1.1- Intelligent Transport Systems (ITS); Security; Trust and Privacy Management; Technical Report. 2012. Available: <http://www.etsi.org/deliver/etsi_ ts/102900_102999/102941/01.01.01_60/ts_102941v010101p.pdf>. Accessed: 01/09/2016. Citations on pages 27 and 55.

Intelligent Transportation Systems (ITS) view. 2012. Available: <http://www.etsi.org/ images/files/membership/ETSI_ITS_09_2012.jpg>. Accessed: 01/09/2016. Citations on pages 31 and 32.

ETSI 103 097. Intelligent transport systems (its); security; security header and certificate formats. technical report. ETSI TS 103097 v1.2.1, p. 1-35, June 2015. Citation on page 77.

FREUDIGER, J.; MANSHAEI, M. H.; BOUDEC, J.-Y. L.; HUBAUX, J.-P. On the age of pseudonyms in mobile ad hoc networks. In: IEEE. INFOCOM, 2010 Proceedings IEEE. [S.1.], 2010. p. 1-9. Citation on page 104.

FREUDIGER, J.; RAYA, M.; FÉLEGYHÁZI, M.; PAPADIMITRATOS, P. et al. Mix-zones for location privacy in vehicular networks. 2007. Citation on page 101.

FUENTES, J. M. d.; GONZÁLEZ-TABLAS, A. I.; RIBAGORDA, A. Overview of security issues in vehicular ad-hoc networks. IGI Global, 2010. Citations on pages 42, 43, 60, and 100.

GERLACH, M. Assessing and improving privacy in vanets. ESCAR, Embedded Security in Cars, 2006. Citations on pages 40 and 104.

GERLACH, M.; GUTTLER, F. Privacy in vanets using changing pseudonyms-ideal and real. In: IEEE. 2007 IEEE 65th Vehicular Technology Conference-VTC2007-Spring. [S.1.], 2007. p. 2521-2525. Citation on page 104.

GROUP, I. W. et al. Standard specification for telecommunications and information exchange between roadside and vehicle systems- 5 ghz band dedicated short range communications (dsrc) medium access control (mac) and physical layer (phy) specifications. ASTM DSR STD E231302, 2002. Citation on page 37.

HAMIDA, E. B.; NOURA, H.; ZNAIDI, W. Security of cooperative intelligent transport systems: Standards, threats analysis and cryptographic countermeasures. Electronics, Multidisciplinary Digital Publishing Institute, v. 4, n. 3, p. 380-423, 2015. Citations on pages 32, 34, 35, 36, and 77.

HAMIEH, A.; BEN-OTHMAN, J.; MOKDAD, L. Detection of radio interference attacks in vanet. In: IEEE. Global Telecommunications Conference, 2009. GLOBECOM 2009. IEEE. [S.1.], 2009. p. 1-5. Citation on page 46. 
HARTENSTEIN, H.; LABERTEAUX, L. A tutorial survey on vehicular ad hoc networks. IEEE Communications magazine, IEEE, v. 46, n. 6, p. 164-171, 2008. Citation on page 38.

HEDGES, C.; PERRY, F. Overview and use of SAE J2735 message sets for commercial vehicles. [S.1.], 2008. Citation on page 40.

HOFFMAN, K.; ZAGE, D.; NITA-ROTARU, C. A survey of attack and defense techniques for reputation systems. ACM Computing Surveys (CSUR), ACM, v. 42, n. 1, p. 1, 2009. Citation on page 79 .

HUBAUX, J.-P.; CAPKUN, S.; LUO, J. The security and privacy of smart vehicles. IEEE Security \& Privacy Magazine, v. 2, n. LCA-ARTICLE-2004-007, p. 49-55, 2004. Citation on page 41 .

IEEE. IEEE Standard for Wireless Access in Vehicular Environments Security Services for Applications and Management Messages. IEEE Std. 1609.2-2013 (Revis. IEEE Std. 1609.2-2006). 2013. Available: <http://www.techstreet.com/ieee/products/preview/1826787>. Accessed: 01/09/2016. Citations on pages 27 and 55.

IEEE 1609.1. Trial-use standard for wireless access in vehicular environments (WAVE) - resource manager. IEEE Std 1609.1, p. 1-71, October 2006. Citation on page 40.

IEEE 1609.2-2006. Ieee standard for wireless access in vehicular environments security services for applications and management messages. IEEE Std 1609.2, p. 1-117, July 2006. Citations on pages 40 and 55 .

IEEE 1609.2-2013. Ieee standard for wireless access in vehicular environments security services for applications and management messages. IEEE Std 1609.2 (Revision of IEEE Std 1609.22006), p. 1-289, April 2013. Citations on pages 40, 55, and 77.

IEEE 1609.3. Trial-use standard for wireless access in vehicular environments (WAVE) - networking services. IEEE Std 1609.3, p. 1-99, April 2007. Citation on page 40.

IEEE 1609.4. Trial-use standard for wireless access in vehicular environments (WAVE) - multichannel operations. IEEE Std 1609.4, p. 1-82, July 2006. Citation on page 39.

JAIMES, L. M. S.; ULLAH, K.; MOREIRA, E. dos S. Ars: Anonymous reputation system for vehicular ad hoc networks. In: IEEE. Communications (LATINCOM), 2016 8th IEEE Latin-American Conference on. [S.1.], 2016. p. 1-6. Citations on pages 53 and 83.

A secure commercial ads dissemination scheme for vehicular networks. In: IEEE. Communications (LATINCOM), 2016 8th IEEE Latin-American Conference on. [S.1.], 2016. p. 1-6. Citation on page 64.

JIANG, D.; DELGROSSI, L. Ieee 802.11 p: Towards an international standard for wireless access in vehicular environments. In: IEEE. Vehicular Technology Conference, 2008. VTC Spring 2008. IEEE. [S.1.], 2008. p. 2036-2040. Citations on pages 38 and 39.

JIN, Y.; KESIDIS, G.; SHIN, J.; KOCAK, F.; YI, Y. Impacts of selfish behaviors on the scalability of hybrid client-server and peer-to-peer caching systems. IEEE/ACM Transactions on Networking, IEEE, v. 23, n. 6, p. 1818-1831, 2015. Citation on page 84.

KAUSHIK, S. S. Review of different approaches for privacy scheme in vanets. Int. J. Adv. Eng. Technol, v. 5, p. 2231-1963, 2013. Citation on page 47. 
KIM, J.-H.; LEE, S. Reliable routing protocol for vehicular ad hoc networks. AEU-International Journal of Electronics and Communications, Elsevier, v. 65, n. 3, p. 268-271, 2011. Citation on page 85 .

KIM, T.-H.; HONG, W.-K.; KIM, H.-C.; LEE, Y.-D. An effective data dissemination in vehicular ad-hoc network. In: SPRINGER. International Conference on Information Networking. [S.1.], 2007. p. 295-304. Citation on page 37.

KOSCH, T.; KULP, I.; BECHLER, M.; STRASSBERGER, M.; WEYL, B.; LASOWSKI, R. Communication architecture for cooperative systems in europe. IEEE Communications Magazine, IEEE, v. 47, n. 5, p. 116-125, 2009. Citation on page 35.

LAUTER, K. The advantages of elliptic curve cryptography for wireless security. IEEE Wireless communications, v. 11, n. 1, p. 62-67, 2004. Citation on page 49.

LEBRUN, J.; CHUAH, C.-N.; GHOSAL, D.; ZHANG, M. Knowledge-based opportunistic forwarding in vehicular wireless ad hoc networks. In: IEEE. Vehicular technology conference, 2005. VTC 2005-Spring. 2005 IEEE 61st. [S.1.], 2005. v. 4, p. 2289-2293. Citation on page 85.

LEE, E.-J.; BAE, I.-H. A reputation-based adaptive trust management system for vehicular clouds. In: SPRINGER. International Conference on Testbeds and Research Infrastructures. [S.1.], 2014. p. 77-86. Citations on pages 43 and 90.

LI, M.; SAMPIGETHAYA, K.; HUANG, L.; POOVENDRAN, R. Swing \& swap: user-centric approaches towards maximizing location privacy. In: ACM. Proceedings of the 5th ACM workshop on Privacy in electronic society. [S.1.], 2006. p. 19-28. Citations on pages 103 and 104.

LI, Q.; MALIP, A.; MARTIN, K. M.; NG, S.-L.; ZHANG, J. A reputation-based announcement scheme for vanets. Vehicular Technology, IEEE Transactions on, IEEE, v. 61, n. 9, p. 40954108, 2012. Citation on page 56.

LI, X.; LIU, J.; LI, X.; SUN, W. Rgte: A reputation-based global trust establishment in vanets. In: IEEE. Intelligent Networking and Collaborative Systems (INCoS), 2013 5th International Conference on. [S.1.], 2013. p. 210-214. Citations on pages 54 and 56.

LI, Z.; CHIGAN, C. Joint privacy and reputation assurance for vanets. In: IEEE. 2012 IEEE International Conference on Communications (ICC). [S.1.], 2012. p. 560-565. Citations on pages 57,86 , and 89 .

LI, Z.; CHIGAN, C. T. On joint privacy and reputation assurance for vehicular ad hoc networks. IEEE Transactions on Mobile Computing, IEEE, v. 13, n. 10, p. 2334-2344, 2014. Citation on page 43.

LIAO, J.; LI, J. Effectively changing pseudonyms for privacy protection in vanets. In: IEEE. 2009 10th International Symposium on Pervasive Systems, Algorithms, and Networks. [S.1.], 2009. p. 648-652. Citations on pages 103, 104, 105, 107, 108, and 109.

LIAO, J.; LI, J.; PAN, Y. Cooperatively changing pseudonyms for privacy protection in vanets. In: Proceedings of the 2nd IEEE international conference on wireless access in vehicular environments (WAVE), Shanghai, China. [S.1.: s.n.], 2009. p. 13-8. Citation on page 104. 
LO, N.-W.; TSAI, H.-C. A reputation system for traffic safety event on vehicular ad hoc networks. EURASIP Journal on Wireless Communications and Networking, Springer International Publishing, v. 2009, n. 1, p. 1, 2009. Citations on pages 89 and 90.

LOCHERT, C.; HARTENSTEIN, H.; TIAN, J.; FUSSLER, H.; HERMANN, D.; MAUVE, M. A routing strategy for vehicular ad hoc networks in city environments. In: IEEE. Intelligent Vehicles Symposium, 2003. Proceedings. IEEE. [S.1.], 2003. p. 156-161. Citation on page 105.

LU, R.; LIN, X.; LUAN, T. H.; LIANG, X.; SHEN, X. Pseudonym changing at social spots: An effective strategy for location privacy in vanets. IEEE transactions on vehicular technology, IEEE, v. 61, n. 1, p. 86-96, 2012. Citation on page 102.

MA, Z.; KARGL, F.; WEBER, M. Pseudonym-on-demand: a new pseudonym refill strategy for vehicular communications. In: IEEE. Vehicular Technology Conference, 2008. VTC 2008Fall. IEEE 68th. [S.1.], 2008. p. 1-5. Citation on page 101.

MALLA, A. M.; SAHU, R. K. Security attacks with an effective solution for dos attacks in vanet. International Journal of Computer Applications, Foundation of Computer Science, v. 66, n. 22, 2013. Citation on page 46.

MÁRMOL, F. G.; PÉREZ, G. M. Trip, a trust and reputation infrastructure-based proposal for vehicular ad hoc networks. Journal of Network and Computer Applications, Elsevier, v. 35, n. 3, p. 934-941, 2012. Citations on pages 48, 89, and 90.

MEJRI, M. N.; BEN-OTHMAN, J.; HAMDI, M. Survey on vanet security challenges and possible cryptographic solutions. Vehicular Communications, Elsevier, v. 1, n. 2, p. 53-66, 2014. Citation on page 43.

MENEGUETTE, R. I.; FILHO, P. G.; GUIDONI, D. L.; PESSIN, G.; VILLAS, L. A.; UEYAMA, $\mathrm{J}$. Increasing intelligence in inter-vehicle communications to reduce traffic congestions: experiments in urban and highway environments. PLoS one, Public Library of Science, v. 11, n. 8, p. e0159110, 2016. Citation on page 36.

MIKKI, M.; MANSOUR, Y. M.; YIM, K. Privacy preserving secure communication protocol for vehicular ad hoc networks. In: IEEE. Innovative Mobile and Internet Services in Ubiquitous Computing (IMIS), 2013 Seventh International Conference on. [S.1.], 2013. p. 188-195. Citation on page 45.

MINHAS, U. F.; ZHANG, J.; TRAN, T.; COHEN, R. A multifaceted approach to modeling agent trust for effective communication in the application of mobile ad hoc vehicular networks. IEEE Transactions on Systems, Man, and Cybernetics, Part C (Applications and Reviews), IEEE, v. 41, n. 3, p. 407-420, 2011. Citation on page 43.

Network Simulation Tools Project Team. Veins simulator. 2016. Available: <https:// networksimulationtools.com/veins-simulator/>. Accessed: 31/10/2016. Citation on page 51.

NOGUEIRA, M.; SILVA, H.; SANTOS, A.; PUJOLLE, G. A security management architecture for supporting routing services on wanets. IEEE Transactions on Network and Service Management, IEEE, v. 9, n. 2, p. 156-168, 2012. Citation on page 47.

PAN, Y.; LI, J. Cooperative pseudonym change scheme based on the number of neighbors in vanets. Journal of Network and Computer Applications, Elsevier, v. 36, n. 6, p. 1599-1609, 2013. Citation on page 104. 
PAN, Y.; LI, J.; FENG, L.; XU, B. An analytical model for random changing pseudonyms scheme in vanets. In: IEEE. Network Computing and Information Security (NCIS), 2011 International Conference on. [S.1.], 2011. v. 2, p. 141-145. Citation on page 104.

PAPADIMITRATOS, P.; BUTTYAN, L.; HOLCZER, T.; SCHOCH, E.; FREUDIGER, J.; RAYA, M.; MA, Z.; KARGL, F.; KUNG, A.; HUBAUX, J.-P. Secure vehicular communication systems: design and architecture. IEEE Communications Magazine, IEEE, v. 46, n. 11, p. 100-109, 2008. Citation on page 79.

PETIT, J.; SHLADOVER, S. E. Potential cyberattacks on automated vehicles. IEEE Transactions on Intelligent Transportation Systems, IEEE, v. 16, n. 2, p. 546-556, 2015. Citation on page 45 .

PRIYA, K.; KARUPPANAN, K. Secure privacy and distributed group authentication for vanet. In: IEEE. Recent Trends in Information Technology (ICRTIT), 2011 International Conference on. [S.1.], 2011. p. 301-306. Citation on page 47.

R, R.; S, S. A survey on security challenges and threats of vehicular adhoc networks(vanets). International Journal of Engineering Research \& Tecnhology (IJERT), v. 3, n. 2, 2014. ISSN 2278-0181. Citation on page 46.

RAWAT, A.; SHARMA, S.; SUSHIL, R. Vanet: Security attacks and its possible solutions. Journal of Information and Operations Management, Bioinfo Publications, v. 3, n. 1, p. 301, 2012. Citations on pages 45 and 47.

RAYA, M.; HUBAUX, J.-P. The security of vehicular ad hoc networks. In: ACM. Proceedings of the 3rd ACM workshop on Security of ad hoc and sensor networks. [S.1.], 2005. p. 11-21. Citations on pages 41, 43, and 49.

Securing vehicular ad hoc networks. Journal of Computer Security, IOS Press, v. 15, n. 1, p. 39-68, 2007. Citations on pages 33 and 35.

RESNICK, P.; KUWABARA, K.; ZECKHAUSER, R.; FRIEDMAN, E. Reputation systems. Communications of the ACM, ACM, v. 43, n. 12, p. 45-48, 2000. Citation on page 48.

ROSELINMARY, S.; MAHESHWARI, M.; THAMARAISELVAN, M. Early detection of dos attacks in vanet using attacked packet detection algorithm (apda). In: IEEE. Information Communication and Embedded Systems (ICICES), 2013 International Conference on. [S.1.], 2013. p. 237-240. Citation on page 46.

ROUFA, R. M. I.; MUSTAFAA, H.; TAYLORA, S. O. T.; XUA, W.; GRUTESERB, M.; TRAPPEB, W.; SESKARB, I. Security and privacy vulnerabilities of in-car wireless networks: A tire pressure monitoring system case study. In: 19th USENIX Security Symposium, Washington DC. [S.1.: s.n.], 2010. p. 11-13. Citation on page 104.

SAE. Dedicated short range communications (dsrc) message set dictionary. SAE J2735, p. 1-359, 2009. Citation on page 40 .

SAI, S.; NIWA, E.; MASE, K.; NISHIBORI, M.; INOUE, J.; OBUCHI, M.; HARADA, T.; ITO, H.; MIZUTANI, K.; KIZU, M. Field evaluation of uhf radio propagation for an its safety system in an urban environment. IEEE Communications Magazine, IEEE, v. 47, n. 11, p. 120-127, 2009. Citation on page 38 . 
SEDJELMACI, H.; SENOUCI, S. M. A new intrusion detection framework for vehicular networks. In: IEEE. 2014 IEEE International Conference on Communications (ICC). [S.1.], 2014. p. 538-543. Citation on page 47.

SOMMER, C.; GERMAN, R.; DRESSLER, F. Bidirectionally Coupled Network and Road Traffic Simulation for Improved IVC Analysis. IEEE Transactions on Mobile Computing, IEEE, v. 10, n. 1, p. 3-15, January 2011. Citation on page 51.

SUMRA, I. A.; AHMAD, I.; HASBULLAH, H. et al. Classes of attacks in vanet. In: IEEE. Electronics, Communications and Photonics Conference (SIECPC), 2011 Saudi International. [S.1.], 2011. p. 1-5. Citation on page 46.

SUMRA, I. A.; HASBULLAH, H.; AHMAD, I. et al. Forming vehicular web of trust in vanet. In: IEEE. Electronics, Communications and Photonics Conference (SIECPC), 2011 Saudi International. [S.1.], 2011. p. 1-6. Citation on page 47.

SUMRA, I. A.; MANAN, J.-L. A.; HASBULLAH, H. Timing attack in vehicular network. In: Proceedings of the 15th WSEAS International Conference on Computers, World Scientific and Engineering Academy and Society (WSEAS), Corfu Island, Greece. [S.1.: s.n.], 2011. p. 151-155. Citation on page 47.

SUN, J.; FANG, Y. Defense against misbehavior in anonymous vehicular ad hoc networks. Ad Hoc Networks, Elsevier, v. 7, n. 8, p. 1515-1525, 2009. Citation on page 53.

TAJEDDINE, A.; KAYSSI, A.; CHEHAB, A. A privacy-preserving trust model for vanets. In: IEEE. Computer and Information Technology (CIT), 2010 IEEE 10th International Conference on. [S.1.], 2010. p. 832-837. Citation on page 57.

TENGSTRAND, S. Ö.; FORS, K.; STENUMGAARD, P.; WIKLUNDH, K. Jamming and interference vulnerability of ieee 802.11 p. In: IEEE. 2014 International Symposium on Electromagnetic Compatibility. [S.1.], 2014. p. 533-538. Citation on page 46.

TOMAR, P.; CHAURASIA, B. K.; TOMAR, G. State of the art of data dissemination in vanets. International Journal of Computer Theory and Engineering, IACSIT Press, v. 2, n. 6, p. 957, 2010. Citation on page 36.

TURNER, S.; HOUSLEY, R.; POLK, T.; BROWN, D. R.; YIU, K. Elliptic curve cryptography subject public key information. 2009. Citation on page 77.

ULLAH, K.; JAIMES, L. M.; YOKOYAMA, R. S.; MOREIRA, E. dos S. Advertising roadside services using vehicular ad hoc network (vanet) opportunistic capabilities. In: in 4th International Conference on Advances in Vehicular Systems, Technologies and Applications. [S.1.: s.n.], 2015. p. 7-13. Citations on pages 42 and 83.

ULLAH, K.; SANTOS, L. M.; MICHELIN, J.; MOREIRA, E. D. S. File transfer in vehicular ad-hoc networks. In: IEEE. Computing Systems Engineering (SBESC), 2013 III Brazilian Symposium on. [S.1.], 2013. p. 175-176. Citation on page 32.

ULLAH, K.; SANTOS, L. M.; RIBEIRO, J. B.; MOREIRA, E. D. Sadp: A lightweight beaconingbased commercial services advertisement protocol for vehicular ad hoc network. In: SPRINGER. International Conference on Ad-Hoc Networks and Wireless. [S.1.], 2016. p. 279-293. Citations on pages 42,81 , and 83 . 
UZCÁTEGUI, R. A.; SUCRE, A. J. D.; ACOSTA-MARUM, G. Wave: A tutorial. IEEE Communications Magazine, IEEE, v. 47, n. 5, p. 126-133, 2009. Citation on page 38.

VANNI, R. M.; SANTOS, L. M.; MAPP, G.; MOREIRA, E. Ontology driven reputation model for vanet. In: IARIA. AICT 2016, The Twelfth Advanced International Conference on Telecommunications. [S.1.], 2016. p. 14-19. Citation on page 58.

VILLAS, L. A.; RAMOS, H. S.; BOUKERCHE, A.; GUIDONI, D. L.; ARAUJO, R. B.; LOUREIRO, A. A. An efficient and robust data dissemination protocol for vehicular ad hoc networks. In: ACM. Proceedings of the 9th ACM symposium on Performance evaluation of wireless ad hoc, sensor, and ubiquitous networks. [S.1.], 2012. p. 39-46. Citation on page 37.

W3C. PROV-O: The PROV Ontology. 2013. Available: <https://www.w3.org/TR/prov-o/>. Accessed: 30/03/2017. Citation on page 58.

WANG, X.; CHENG, W.; MOHAPATRA, P.; ABDELZAHER, T. Artsense: Anonymous reputation and trust in participatory sensing. In: IEEE. INFOCOM, 2013 Proceedings IEEE. [S.1.], 2013. p. 2517-2525. Citation on page 61.

WEI, Y.-C.; CHEN, Y.-M. Efficient self-organized trust management in location privacy enhanced vanets. In: SPRINGER. International Workshop on Information Security Applications. [S.1.], 2012. p. 328-344. Citation on page 43.

WOLF, M. Vehicular security mechanisms. In: Security Engineering for Vehicular IT Systems. [S.1.]: Springer, 2009. p. 121-165. Citation on page 46.

WU, Q.; DOMINGO-FERRER, J.; GONZÁLEZ-NICOLÁS, U. Balanced trustworthiness, safety, and privacy in vehicle-to-vehicle communications. IEEE Transactions on Vehicular Technology, IEEE, v. 59, n. 2, p. 559-573, 2010. Citations on pages 28 and 43.

XIAO, B.; YU, B.; GAO, C. Detection and localization of sybil nodes in vanets. In: ACM. Proceedings of the 2006 workshop on Dependability issues in wireless ad hoc networks and sensor networks. [S.1.], 2006. p. 1-8. Citation on page 46.

YANG, F.; WANG, S.; LI, J.; LIU, Z.; SUN, Q. An overview of internet of vehicles. China Communications, IEEE, v. 11, n. 10, p. 1-15, 2014. Citations on pages 37 and 54.

YANG, N. A similarity based trust and reputation management framework for vanets. International Journal of Future Generation Communication and Networking, v. 6, n. 2, p. 25-34, 2013. Citation on page 40.

YOKOYAMA, R.; KIMURA, B.; JAIMES, L.; MOREIRA, E. A beaconing-based opportunistic service discovery protocol for vehicular networks. In: Advanced Information Networking and Applications Workshops (WAINA), 2014 28th International Conference on. [S.1.: s.n.], 2014. p. 498-503. Citation on page 83.

ZEADALLY, S.; HUNT, R.; CHEN, Y.-S.; IRWIN, A.; HASSAN, A. Vehicular ad hoc networks (vanets): status, results, and challenges. Telecommunication Systems, Springer, v. 50, n. 4, p. 217-241, 2012. Citation on page 39.

Vehicular ad hoc networks (vanets): status, results, and challenges. Telecommunication Systems, Springer, v. 50, n. 4, p. 217-241, 2012. Citations on pages 44, 45, 46, and 47. 
ZHANG, J. A survey on trust management for vanets. In: IEEE. 2011 IEEE International Conference on Advanced Information Networking and Applications. [S.1.], 2011. p. 105112. Citations on pages 36,47 , and 48 .

ZHANG, J.; SENSOY, M.; COHEN, R. A detailed comparison of probabilistic approaches for coping with unfair ratings in trust and reputation systems. In: IEEE. Privacy, Security and Trust, 2008. PST'08. Sixth Annual Conference on. [S.1.], 2008. p. 189-200. Citation on page 48. 


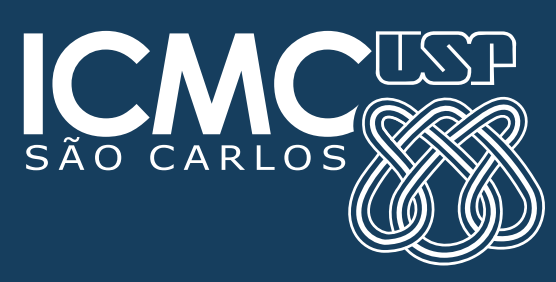

